\title{
Smooth Signed Distance Field Textures
}

\author{
by
}

\section{Ehren Choy,}

\author{
A thesis submitted to the \\ Faculty of Graduate and Postdoctoral Affairs \\ in partial fulfillment of the requirements for the degree of
}

\section{Master of Computer Science}

Ottawa-Carleton Institute for Computer Science

The School of Computer Science

Carleton University

Ottawa, Ontario

April, 2016

(C)Copyright

Ehren Choy, 2016 
The undersigned hereby recommends to the

Faculty of Graduate and Postdoctoral Affairs acceptance of the thesis

\title{
Smooth Signed Distance Field Textures
}

\author{
submitted by Ehren Choy, \\ in partial fulfillment of the requirements for the degree of
}

Master of Computer Science

Professor David Mould, Thesis Supervisor

Professor Oliver van Kaick, School of Computer Science

Professor Eric Dubois,

School of Electrical Engineering and Computer Science

Professor Anil Maheshwari, Chair, School of Computer Science

Ottawa-Carleton Institute for Computer Science

The School of Computer Science

Carleton University

April, 2016 


\section{Abstract}

We present a procedural method to produce different types of organic texture. We create a texture by defining a mesh over the texture domain, with each point of the mesh being assigned a label. We group the points of the mesh, with each group consisting of points which all share the same label, and are all connected to each other in the mesh. An implicit region is created for every group.

To define a region, we derive the interior and exterior contour. The interior contour connects outer points of a group together, while the exterior contour connects points that are neighbours of that group. The region is defined to be between the interior and exterior contour. We construct a smooth signed distance field for each contour, and blend the fields of the contours together to form the region. Wherever one distance field is discontinuous, we use the distance field of the opposing contour to define the region. A texture is created by blending together the regions from all groups.

Our work makes the following contributions. We propose the idea of defining an implicit field by using the smooth signed distance fields of two sets of boundary line segments. By using two sets of line segments, we are able to produce a single implicit field that is everywhere smooth. We apply this idea to the generation of organic texture. Our method of texture generation is distinguished from previous techniques by being able to create resolution-independent texture, such that texture elements can be smooth or irregularly shaped. We have found our method suitable for modeling many types of patterns found in the natural world, including patterns commonly found in frogs and lizards. 
This thesis is dedicated to my parents, Robin 8 Marie Choy, for their endless love and encouragement. 


\section{Acknowledgments}

My deepest gratitude must first be given to my advisor Professor David Mould. Throughout all the challenges and setbacks, his guidance and encouragement has made my academic journey a memorable one. From his lighthearted humour to his inspirational ideas, he has played a central role in the development of my research. Not often do I have the opportunity to show my appreciation, but I am glad to do so here.

I would also like to thank my defense committee, Professor Oliver van Kaick and Professor Eric Dubois, for providing their comments and suggestions. They have helped greatly in improving the presentation of my work.

Lastly, I must thank all my lab members in HP 5317. I am glad to have shared so many long conversations, both serious and mundane, with them. Their kindness was a consistent source of support, and I will remember the time spent with them fondly. 


\section{Table of Contents}

Abstract $\quad$ iii

Acknowledgments $\quad$ v

Table of Contents $\quad$ vi

List of Tables $\quad$ X

List of Figures $\quad$ xi

1 Introduction 1

1.1 Goal ................................. 2

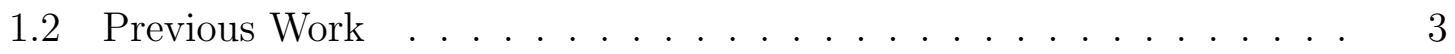

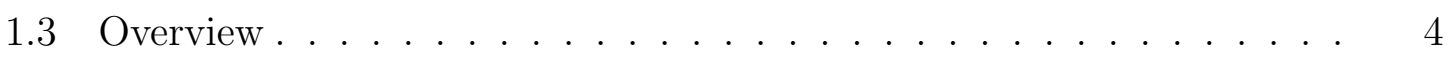

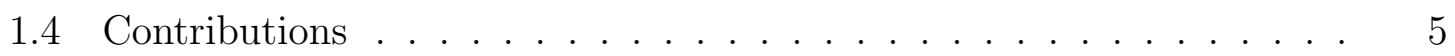

2 Procedural Texture Functions $\quad 6$

2.1 Describing Texture . . . . . . . . . . . . . . . . . 6

2.2 Evaluating Procedural Texture . . . . . . . . . . . . . 8

2.3 Procedural Noise Functions . . . . . . . . . . . . . . . . . . . . . . 9

2.3.1 Perlin Noise . . . . . . . . . . . . . . . . . 10

2.3.2 Sparse Convolution and Gabor Noise . . . . . . . . . . . . . . 11

2.4 Texture Basis Functions . . . . . . . . . . . . . . . . . . 12

2.4.1 Worley Noise . . . . . . . . . . . . . . . . . 12

2.4.2 Partition of Unity Textures . . . . . . . . . . . . . . 13

2.5 Reaction Diffusion Textures . . . . . . . . . . . . . . . . 15

2.6 Exemplar-Based Textures . . . . . . . . . . . . . . . . . . . . 17

2.6.1 Pixel-Based . . . . . . . . . . . . . . . . . 18 
2.6.2 Patch-Based . . . . . . . . . . . . . . . . . . . . . . . 19

2.6 .3 Optimization-Based . . . . . . . . . . . . . . . 20

2.6.4 Surface Texture Synthesis _ . . . . . . . . . . . . 20

2.6 .5 Extensions . . . . . . . . . . . . . . . . . . . . 21

2.7 Implicit Modeling Methods . . . . . . . . . . . . . . . . . . . 22

2.8 Smooth Distance Field Textures . . . . . . . . . . . . . . . . . . 23

3 Smooth Signed Distance Functions $\quad 25$

3.1 Definition . . . . . . . . . . . . . . . . . . 25

3.2 Normalized Approximation . . . . . . . . . . . . . . . . . . 27

3.3 Interpolation-Based Methods . . . . . . . . . . . . . . . 27

3.3.1 Inverse-Distance Interpolation . . . . . . . . . . . . . . 28

3.3.2 Interpolation Framework . . . . . . . . . . . . . . . . . 28

3.3 .3 Radial Basis Functions . . . . . . . . . . . . . . . . . . . 30

3.4 Potential-Based Methods . . . . . . . . . . . . . . . . . . . . . . 32

3.5 Discrete Methods . . . . . . . . . . . . . . . . . . . . . . . . . 33

3.6 Constructive Methods . . . . . . . . . . . . . . . . . . . 34

4 Smooth Signed Distance Field Textures $\quad 38$

4.1 Algorithm . . . . . . . . . . . . . . . . . . . . . . . . . . . 39

4.1 .1 Mesh Generation . . . . . . . . . . . . . . . . 40

$4.1 .2 \quad$ Label Assignment . . . . . . . . . . . . . . . . . . . . . 41

4.1 .3 Point Grouping . . . . . . . . . . . . . . . . . . . . 41

4.1 .4 Contour Definition . . . . . . . . . . . . . . . 42

$4.1 .5 \quad$ Field Creation . . . . . . . . . . . . . . . . . 45

4.1 .6 Region Merging . . . . . . . . . . . . . . . . . . . . 49

4.2 Blending . . . . . . . . . . . . . . . . . . . . 51

4.3 Offset Distance . . . . . . . . . . . . . . . . . . . . . 52

4.4 Labeling . . . . . . . . . . . . . . . . . . . . . 54

4.4 .1 Stripes and Spots . . . . . . . . . . . . . . . 54

4.4 .2 Graph Traversal . . . . . . . . . . . . . . . . . . 54

4.5 Point Distribution . . . . . . . . . . . . . . . . . 56

4.6 Color Values . . . . . . . . . . . . . . . . . . . . . . . . . 58

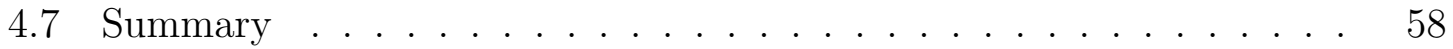


$\begin{array}{llr}5 & \text { Results } & 61\end{array}$

5.1 Introduction . . . . . . . . . . . . . . . . . 62

5.2 Method Inputs . . . . . . . . . . . . . . . . . . . 63

5.3 Irregular Patterns . . . . . . . . . . . . . . . . . . . 64

5.3.1 Irregular Spot Texture . . . . . . . . . . . . . . . . . 64

5.3 .2 Irregular Stripe Texture . . . . . . . . . . . . . . . 65

5.4 Smooth Patterns . . . . . . . . . . . . . . . . 66

5.4.1 Rounded Spot Texture . . . . . . . . . . . . . . . 66

5.4.2 Smooth Stripe Texture . . . . . . . . . . . . . . . . . 68

5.5 Voronoi Patterns . . . . . . . . . . . . . . . . 70

5.5.1 Voronoi Definition . . . . . . . . . . . . . 70

5.5.2 Irregular Voronoi Patterns . . . . . . . . . . . . . . . . . 71

5.5.3 Smooth Voronoi Patterns . . . . . . . . . . . . . . . . 72

5.6 Complex Patterns . . . . . . . . . . . . . . . . 73

5.6.1 Image Texture . . . . . . . . . . . . . . . 73

5.6.2 Non-Stationary Texture . . . . . . . . . . . . . . 74

5.6.3 Boundary Constraints . . . . . . . . . . . . . . . 77

5.7 Summary . . . . . . . . . . . . . . . . . . . . 77

$\begin{array}{llr}6 & \text { Discussion } & 79\end{array}$

6.1 Rationale ........................ 79

6.1 Mesh Texture . . . . . . . . . . . . . . . 79

6.1 .2 Field Definition . . . . . . . . . . . . . . 80

6.1.3 Texture Synthesis . . . . . . . . . . . . . . . . . . 82

6.1.4 Comparison .................... 84

6.2 Storage and Performance . . . . . . . . . . . . . . 85

6.2.1 Random Access . . . . . . . . . . . . . . . . . . . . . . 87

6.2.2 Fixed Resolution . . . . . . . . . . . . . . . . 87

6.2.3 Field Value Computation . . . . . . . . . . . . . . 88

6.3 Summary . . . . . . . . . . . . . . . . . 89

7 Conclusion $\quad 91$

7.1 Summary . . . . . . . . . . . . . . . . . . . 91

7.2 Future Work . . . . . . . . . . . . . . . . . . . . 92 
List of References 


\section{List of Tables}

6.1 Timing results for our algorithm for a binary texture $(400 \times 400)$. . 89

6.2 Timing results for our algorithm for different resolutions. . . . . . . 90 


\section{List of Figures}

1.1 Examples of inspirational biological patterns. (a) toad [24], (b) zebra [20], (c) blue poison arrow frog [66], (d) leopard [56], (e) lion fish $[73],(\mathrm{d}) \operatorname{lizard}[61] \ldots \ldots \ldots$. . . . . . . . . . . . . . . 2

1.2 Texture creation using our algorithm. Given a labeled mesh (a), we derive two sets of line segments (b). The created texture is shown in

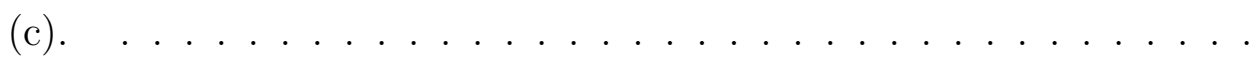

2.1 Examples of patterns with different characteristics. The pattern found in the leopard gecko [81] (a) is stationary and appears similar under translation, while the butterfly [51] (b) is an example of a nonstationary pattern. The zebra pattern [21] (c) is an example of an anisotropic pattern. . . . . . . . . . . . . 6

2.2 Examples of Worley noise . . . . . . . . . . . . . . . . 13

2.3 Behaviour of partition-of-unity textures. (a) texture created by assigning a random color to each point, and in (b) the support of a feature point is shown as a red outline, and the underlying graph is shown in blue.

2.4 Examples of reaction-diffusion . . . . . . . . . . . . . . .

3.1 A signed distance field of an infinite line (a) is intersected with the signed distance field of a circular field (b) producing a smoothed field (c). Isocontours of the produced fields are shown on the bottom row.

3.2 Joining implicit fields. (a) The smooth distance field of a polygon, and (b) isocontours of the produced field. . . . . . . . . . . . .

4.1 From left to right, top to bottom, the six major steps of our algorithm: (a) mesh generation, (b) label assignment, (c) point grouping, (d) contour definition, (e) field creation, and (f) region merging. Each field created in step (e) is combined together to form the texture in step (f). 
4.2 Initial steps of our method. (a) Mesh generation (b) Label Assignment (c) Point Grouping . . . . . . . . . . . . . . . . . . . . . . . . . . . 42

4.3 Interior and exterior contours for a set of points. The interior contour is shown in black, and the exterior contour is shown in gray. The exterior contour consists of two sets of connected line segments, and a single point.

4.4 Polygonal regions of the interior points of a mesh, with regions highlighted in gray. (a) Labeled Mesh (b) Region $\Omega_{I}$ (c) Region $\Omega_{E}$. . .

4.5 Graphical representation of $d_{I}(p)$ and $d_{E}(p)$ for the interior points of a simple mesh, with the corresponding exact distance fields shown as dashed lines. The cross-section area is shown as a dashed line on the mesh image. . . . . . . . . . . . . . . . . . .

4.6 Smooth signed distance field of the interior contour for the interior points of a mesh . . . . . . . . . . . . . . . . .

4.7 Smooth signed distance field of the exterior contour for the interior points of a mesh . . . . . . . . . . . . . . . .

4.8 Cross-section of $d_{M}(p)$ and $d_{N}(p)$ for the interior points of a simple mesh, with the exact distance field midway between contours shown as a dashed line. The cross-section area is shown as a dashed line on the mesh image. . . . . . . . . . . . . . . . . . . 48

4.9 Distance field $d_{M}(p)$ with gradient discontinuities. . . . . . . . . . . 50

4.10 Distance field $d_{N}(p)$ that is $C^{1}$ continuous. . . . . . . . . . . . 50

4.11 Merging implicit regions to form a textures. (a) simple texture, and (b) random binary texture. . . . . . . . . . . . . . . . 51

4.12 Blending of implicit regions with different blending radius $r$. . . . . 53

4.13 Cross-section of $w_{i}(p)$ with different blending radii. The cross-section area is shown as a dashed line on the mesh image. . . . . . . . . . 53

4.14 Different offset distances $K$ to expand or contract a region. . . . . . .

4.15 Cross-section of $d_{N}(p)$ showing different offset distances for a simple mesh. The cross-section area is shown as a dashed line on the mesh

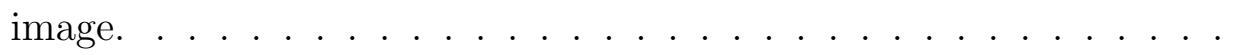

4.16 Stripe and spot patterns created with a graph-based traversal. (a) spot patterns, and (b) stripe patterns. 
4.17 Branching structures created using a graph-based traversal. (a) texture with initial set of labeled points, and (b) texture with labeled points connected to form a minimum spanning tree. . . . . . . . . . . . . . 57

4.18 Random binary textures with varying point densities . . . . . . . . 59

4.19 Different textures generated by varying the point distribution. . . . . 59

4.20 Textures produced with a color spline to produce an outline. (a) initial texture image, (b) discontinuous color transition, and (c) smooth color

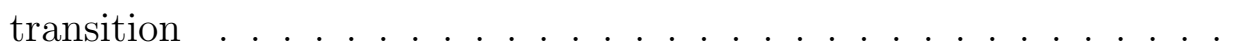

5.1 From left to right, top to bottom, examples of patterns found in the natural world: (a) spotted marsh frog [80], (b) strawberry poisondart frog [34], (c) clown triggerfish [28], (d) emperor angelfish [39], (e) pufferfish [19], and (f) common giraffe [53]. . . . . . . . . . .

5.2 Irregular spot and stripe patterns. (a) irregular spot pattern created with $d=0.10$ and $k=4$ (b) irregular stripe pattern created with

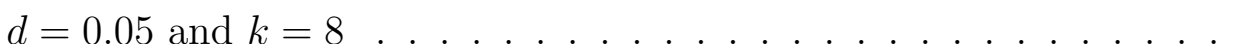

5.3 Irregular spot patterns each with varying point densities $d$ and cluster sizes $k$.

5.4 Irregular stripe patterns each with varying maximum stripe length $k$. 66

5.5 Bend in an irregular stripe pattern. (a) stripe pattern created with $k=8$ and $d=0.10$, and (b) region in the texture with sharp bend. .

5.6 (a) example of a rounded spot texture, and (b) example of smooth stripe pattern.

5.7 Parameters dependent on smooth distance field. (a) smooth distance field of a single line segment, and (b) implicit field of texture with varying smooth distance fields. . . . . . . . . . . . . . . .

5.8 Examples of Voronoi patterns. (a) example of an irregular Voronoi pattern, and (b) example of a smooth Voronoi pattern. . . . . . . . . 70

5.9 Irregular Voronoi patterns each with varying point densities $d$ and

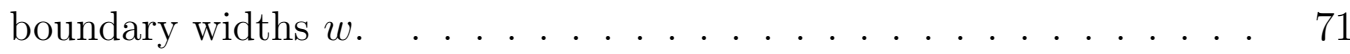

5.10 Examples of different smooth Voronoi patterns. . . . . . . . . . . . . 72

5.11 Examples of complex patterns found in nature: (a) maze coral [10], (b) blue poison arrow frog [40], and (c) lizard [7] . . . . . . . . . . 73

5.12 Example of maze texture. (a) maze texture, and (b) corresponding binary image. . . . . . . . . . . . . . . . . . . . . . 74 
5.13 Examples of textures created from images $[5,45,67]$. (a) original image, and (b) texture produced using probabilistic assignment of labels from image. . . . . . . . . . . . . . . . . . 75

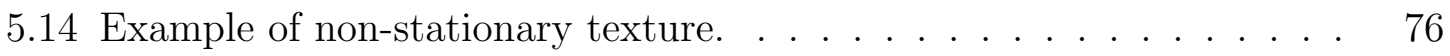

5.15 Example of non-stationary labeling. . . . . . . . . . . . 76

5.16 Texture with single set of boundary points. . . . . . . . . 78

5.17 Texture with two sets of boundary points. . . . . . . . 78

6.1 Bulging in a smooth distance field. (a) Single line segment smooth distance field. (b) Bulge formed by joining two separate line segments. 80

6.2 Analysis of bulging problem. (a) Implicit field of two joined line segments. (b) Gradient magnitude of joined line segments. . . . . . . . . 81

6.3 Implicit field created using an interior and exterior contour. . . . . . 83

6.4 Implicit field created using a single set of line segments. . . . . . . . . 83

6.5 Texture with rotated interior contour. . . . . . . . . . . . . . . 84

6.6 Examples of patterns created using partition of unity textures. . . . 86

6.7 Examples of patterns created using reaction diffusion. . . . . . . 86

6.8 Examples of patterns created using our method. . . . . . . . . . . 86 


\section{Chapter 1}

\section{Introduction}

Textures are used extensively in computer graphics to add visual details onto synthetic images. A texture, or texture map, is an image that is mapped onto the surface of an object to modify surface properties such as color, reflection, transparency, or displacement. Although any arbitrary image can be referred to as a texture, we use a more narrow definition, adapted from Wei [87] and Lewis [49]:

Textures are defined as images, either visual or tactile, consisting of repeating elements forming patterns. Texture can be described through its aggregate characteristics, rather than explicit specification of its elements.

Given this refined definition, a major challenge is how to efficiently create high-quality textures for use in objects of a generated scene.

Procedural texture functions are automatic methods for generating texture that can be solely described through algorithms or mathematical functions. These functions do not rely on artists providing digital photographs or samples, but instead create textures through modifiable parameters. Different procedural texture functions are used depending on the type of texture to be created.

Our work focuses on generating textures that resemble organic patterns found in the natural world. Textures we wish to generate include the various spots and stripes found in the animal kingdom. Within this collection of patterns, there is a tremendous variety in color, shape, and size. Patterns range from the black-and-white stripes of a zebra, to the complex skin patterns found in certain toads. We show several examples of organic patterns in Figure 1.1.

There are several advantages for creating texture using procedural texture functions. We can describe texture as a whole, rather than having to specify a particular 


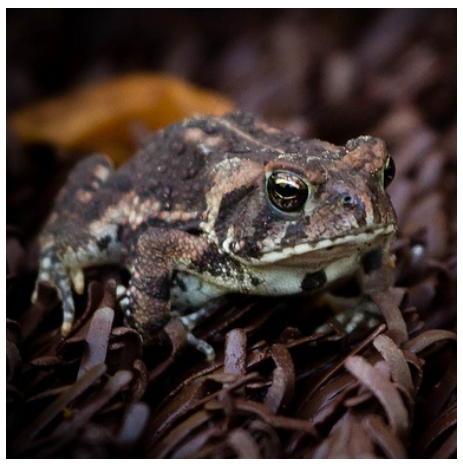

(a)

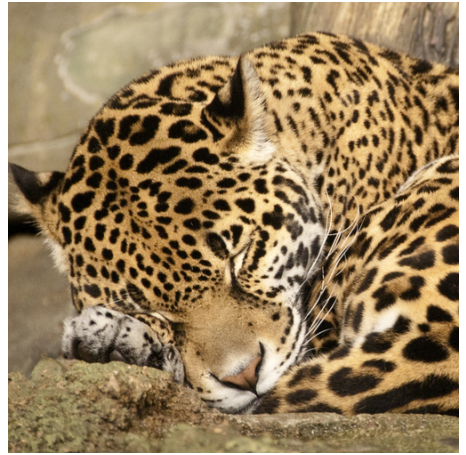

(d)

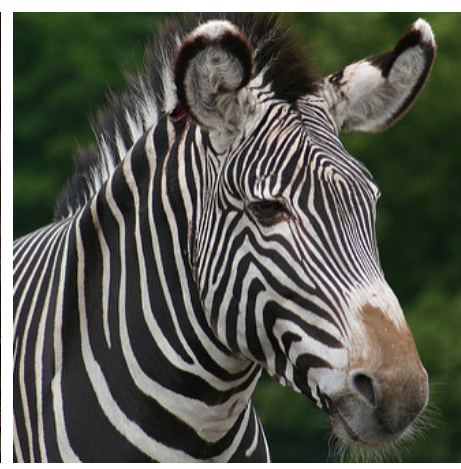

(b)

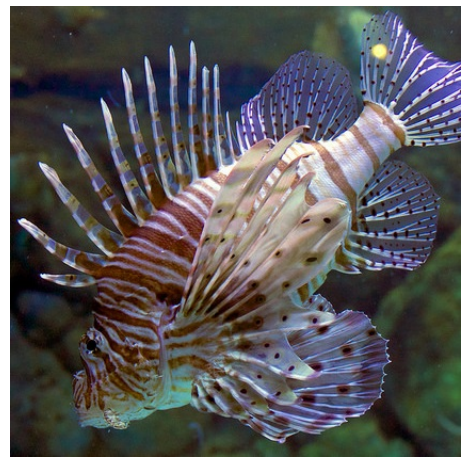

(e)

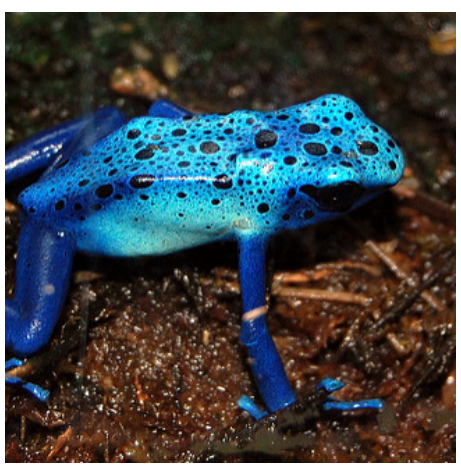

(c)

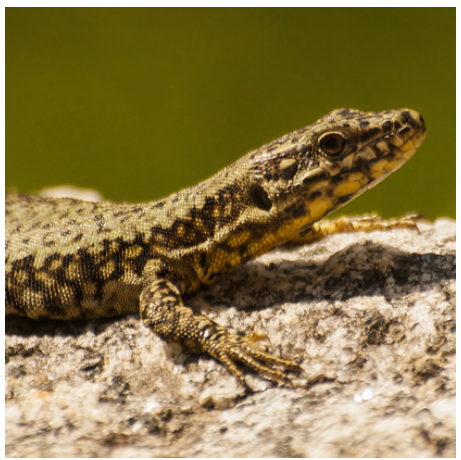

(f)

Figure 1.1: Examples of inspirational biological patterns. (a) toad [24], (b) zebra [20], (c) blue poison arrow frog [66], (d) leopard [56], (e) lion fish [73], (d) lizard [61]

spot or stripe in an image. Modifiable parameters allow artists to gradually change texture appearance, a benefit known as parametric control [25].

Procedural texture functions also provide the ability to discover and synthesize new textures which may not necessarily exist in photographs. Although not necessarily true for all methods, other potential benefits of procedural texture functions include being compact, efficient, and continuous. We discuss the possible benefits of procedural texture functions in detail in Section 2.1.

\subsection{Goal}

Our goal is to create a procedural texture function capable of automatically generating a large class of organic patterns, including certain types of amphibian skin and animal coat markings. The control parameters should be intuitive for a designer, and it 
should be possible to gradually change a texture once produced. Our method should also provide the traditional benefits of procedural texture functions, including being efficient and having a compact and continuous representation.

\subsection{Previous Work}

Texture synthesis is a topic that has been well studied. There are many methods, both procedural and non-procedural, that can be used to produce texture. Methods are distinguished from one another by how they are used, and by the types of textures they generate.

Noise-based techniques (Section 2.3), such as the popular Perlin noise, have parameters which modify the spectral properties of the texture. These techniques generate band-limited random patterns, which can be used to add additional detail to the surface of an object. Reaction-diffusion textures (Section 2.3) operate through a biological simulation on a discrete grid, and are specified through non-linear partial differential equations. The chemical concentrations of reactants are mapped onto colors to produce texture.

Other procedural texture functions use point primitives to specify texture. Worley noise (Section 2.4.1) operates by taking linear combinations of distances from points, and produces Voronoi-like patterns. Partition-of-unity textures (Section 2.4.2) uses points in a mesh to define a set of smooth convex fields.

Many non-procedural methods can also be used to create texture. Exemplarbased methods (Section 2.6) use a given sample image to produce an arbitrarily large texture. Artists can also manually design a texture by using types of implicit surfaces, such as using skeletal implicit surfaces or variational surfaces. Although time-consuming to manually specify, an artist would have complete control over the output texture.

Our method is distinguished from previous approaches by being able to generate texture that is continuous, and that can contain smooth and non-convex texture elements. A comparison between our method, Worley noise, and partition of unity textures is given in Section 6.1.4. 


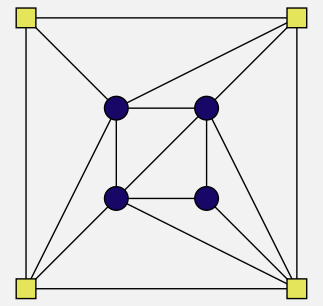

(a) Mesh generation

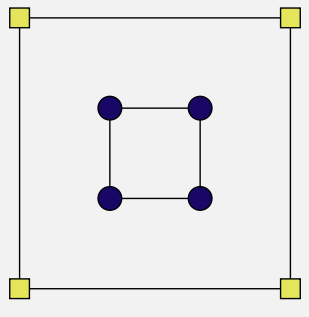

(b) Contour definition

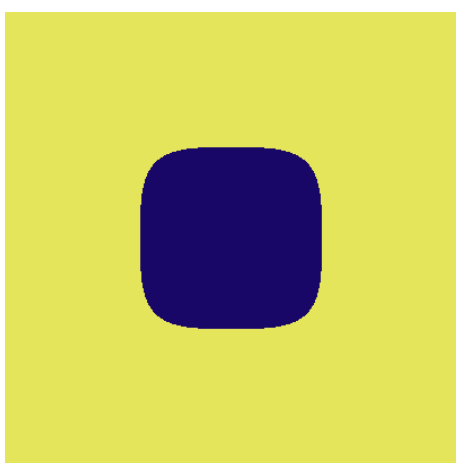

(c) Output texture

Figure 1.2: Texture creation using our algorithm. Given a labeled mesh (a), we derive two sets of line segments (b). The created texture is shown in (c).

\subsection{Overview}

Our method creates a texture by first generating a mesh over the texture domain. Each point of the mesh is then labeled with a color value. Once labels are assigned to the points of the mesh, we divide the points into different groups. Each group consists of points which all share the same label, and are all connected to each other in the mesh. An implicit region will be created for every group.

To define an implicit region, we derive two different sets of mesh edges from each group of points. The two sets of edges define the interior and exterior contour. The interior contour connects outer points of the group together, while the exterior contour connects points that are neighbours of the group. The implicit region is defined to be between the interior and exterior contour, as illustrated in Figure 1.2.

We construct a smooth signed distance field for each contour, and blend the fields of the contours together to form the implicit region. By using a pair of contours, rather than using a single set of line segments, we are able to create an implicit field that is smooth everywhere. Wherever one distance field is discontinuous, we use the distance field of the opposing contour to define the region. A texture is created by blending together the implicit fields from all connected components.

Because regions are defined using smooth implicit fields, we can blend different regions together, and can also expand or contract each texture region. We demonstrate the effectiveness of our algorithm by generating a variety of textures evident in the natural world. 


\subsection{Contributions}

Our work makes the following two contributions:

- We propose the idea of defining a smooth implicit region by using the smooth signed distance fields of two sets of boundary line segments. By using such an approach, we are able to produce regions whose implicit fields are everywhere smooth.

- We present a procedural method for texture synthesis, where texture elements can be rounded or irregularly shaped. Our method is suitable for modeling many types of patterns found in the natural world, including patterns commonly found in frogs and lizards. 


\section{Chapter 2}

\section{Procedural Texture Functions}

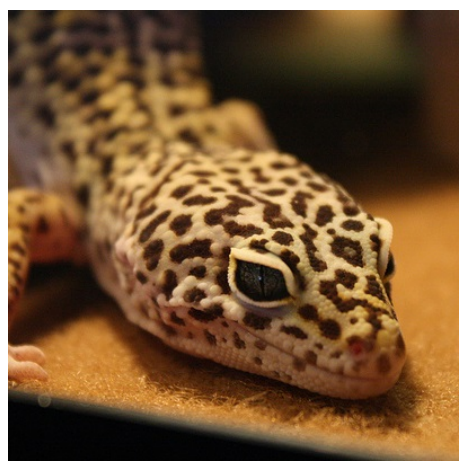

(a)

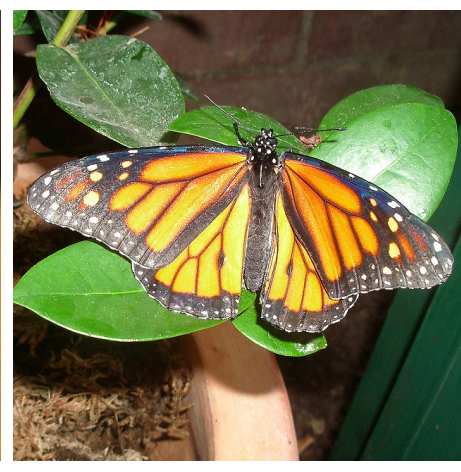

(b)

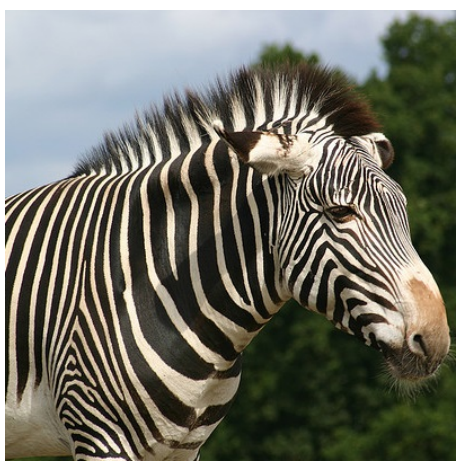

(c)

Figure 2.1: Examples of patterns with different characteristics. The pattern found in the leopard gecko [81] (a) is stationary and appears similar under translation, while the butterfly [51] (b) is an example of a non-stationary pattern. The zebra pattern [21] (c) is an example of an anisotropic pattern.

\subsection{Describing Texture}

We have previously described textures as images containing repeating patterns. This definition is simple, but includes many different types of phenomena. Images of brick walls, marble tiles, and animal markings are all examples of textures. To further distinguish between textures, we use the following list of texture properties.

Stationarity - We say that a texture is stationary if statistical properties of the texture do not vary under translation. That is, given any subset of a texture of 
a particular size, the subset appears to come from the same underlying process. Repeating spot patterns, where spots are distributed uniformly and possess the same size, are stationary patterns. The wing patterns of many butterflies are non-stationary (Figure 2.1), as the size of a butterfly spot varies depending on distance to the boundary of the wing.

Isotropy - We say that a texture is isotropic if rotating a texture does not change the statistical qualities of the texture. Many stripe patterns are not isotropic, but are anisotropic because all stripes would follow a particular direction in the texture. For example, zebra stripes (Figure 2.1) typically run vertically along the zebra body.

Periodicity - We say that a texture is periodic if there exists a pattern which repeats at a regular interval, and where the interval is known as the period. A checkerboard pattern is an example of a pattern which is periodic. Latticebased noise functions (Section 2.3) exhibit periodic artifacts which are caused by interpolation from an underlying grid structure.

Regularity - Closely related to the concept of periodicity is regularity, referring to the perceived randomness of a texture. Stochastic textures are generated by a purely random process, while regular patterns have high degrees of symmetry or periodicity.

Smoothness - Smoothness refers to the continuity of a texture across its domain, where continuity can be described by the existence of derivatives at a point. We say a texture is $C^{k}$ continuous if all derivatives up to the $k$ th order exist and are continuous for any point in the domain. For many amphibian patterns, smooth textures are desirable because there is a gradual transition between colors which should be reflected by the continuity of the texture. Our work produces textures that are $C^{1}$ continuous. Smoothness may also refer to continuity of derivatives along a boundary curve; the path of a circle is smooth, while for a square it is not.

Spectral Density - An alternative description of texture can be found in the frequency domain, where an image is described in terms of a phase and magnitude over a range of frequencies. Procedural noise functions (Section 2.3) operate 
by generating band-limited random patterns. Because the phase of these functions are random, noise functions can be fully characterized by the frequency magnitude or spectral density.

We will later use these definitions in describing procedurally generated texture. We will next consider how to evaluate these procedural methods.

\subsection{Evaluating Procedural Texture}

Different procedural texture functions are used depending on the type of texture that must be generated. Because of the differing goals of these automatic methods, there is no general rubric for evaluation. However, there are a set of criteria that are universally desirable, and which we describe below. This list is based on characteristics described by Ebert et al. [25].

Compact - The texture function should use a small amount of memory. In the ideal case, the memory usage of a texture function should be independent of the size of the texture. In many cases, storage is dependent on the complexity and size of the generated texture.

Efficient - The texture should be generated quickly and should not be computationally expensive. This is especially important for procedural textures, as the texture function must be evaluated many times at different locations in a render.

Continuous - The texture function should be continuous and not dependent on a grid discretization. Simulation-based methods like reaction-diffusion, and many exemplar-based texture methods produce a discrete texture which must later be interpolated to produce a continuous function.

General - The texture function can be parameterized to generate a wide class of patterns and phenomena. Ideally, the parameterization should be continuous to allow an artist to gradually change the texture. Worley noise (Section 2.4.1) is parameterized by the choice of distance functions used for computation, and Gabor noise (Section 2.3.2) provides control over the orientation, principal frequency, and bandwidth of the produced noise. 
Random Access - The value of a texture at a point can be evaluated in constant time, independent of previous evaluations. Simulation-based methods like reaction-diffusion, and many exemplar-based texture methods, do not allow random access of the texture; the entire texture must be evaluated at once.

Surface Parameterized - If a texture is created as an rectangular image in $\mathbb{R}^{2}$, it must be mapped onto the surface of the object for rendering. For some surfaces, there is a natural mapping from texture space to model space. However, textures generally undergo distortion in this mapping process. Rather than having to perform this mapping, it would be desirable if a texture function is defined directly on the surface of the rendered object.

The remainder of this section reviews existing methods for generating texture. We first discuss procedural noise functions, and describe in detail the popular Perlin noise and Gabor noise functions. We next explore existing methods for creating organic patterns including reaction-diffusion textures, Worley noise, and partition of unity textures. We then deviate from procedural methods to discuss exemplar-based texture and implicit modeling methods. Exemplar-based methods are not procedural texture functions, but are still widely used in producing texture. For each method, we describe the attributes that generated textures possess, and how well it matches the above desirable properties.

We conclude this section by framing our method in the context of existing procedural texture functions. This section assumes that the textures are generated in $\mathbb{R}^{2}$, although many of the described methods directly generalize to three dimensions.

\subsection{Procedural Noise Functions}

We start our discussion on existing texture methods with procedural noise functions. Procedural noise functions operate by generating random sequences of numbers, and this randomness is used to perturb a regular pattern (such as a solid color image) to give the final texture. The best-known procedural noise function is Perlin Noise [63], which can be used to generate several different types of patterns such as marble, clouds, and fire.

To effectively perturb a regular pattern, procedural noise functions aim to produce texture which is stationary, isotropic, non-periodic, and stochastic. To control the 
range of perturbation, the range of the noise function should be bounded to some fixed interval (such as -1 to 1 ).

A possible, but insufficient, method of perturbation is to use a pseudorandom number to modify every sample of the original pattern. This approach is insufficient when we consider rendering the pattern onto a surface; slight changes in the scene result in different samples to be chosen in the pattern, leading to a completely different texture. A sequence of random uncorrelated numbers is known as white noise; perturbing images using white noise creates textures with arbitrary and uncontrollable detail.

Described in the frequency domain, white noise has an expected constant power spectrum, whereas we would like procedural noise functions to produce noise only within a prescribed range of frequencies. Noise functions are distinguished by their spectral properties; Perlin noise has been described as "an approximation to white noise band-limited to a single octave" [62].

\subsubsection{Perlin Noise}

We now describe the implementation of Perlin noise [63]. The method is initialized by creating an integer grid of fixed resolution, and assigning a random unit vector to each grid point. The noise value at some location $p$ is obtained using the four corners of the grid cell that contains $p$.

Let $x_{i}$ be a grid point belonging to the cell that contains $p$, and let $\overrightarrow{g_{i}}$ be the unit vector associated with $x_{i}$. The contribution $f_{i}(p)$ of the grid point $x_{i}$ is defined as

$$
f_{i}(p)=\left(p-x_{i}\right) \cdot \overrightarrow{g_{i}}
$$

The final noise value is obtained using an interpolation of the contribution from the four corners of the cell. A spline interpolation, rather than a direct bilinear interpolation, is used to create an everywhere smooth texture.

The original Perlin noise function has since been improved in a later work by Perlin [64]. The original spline produced texture continuous only to its first derivative, and visible discontinuities existed on a noise-displaced surface when shaded (as the surface normal is a derivative operator). Perlin improved the noise function by increasing the order of the polynomial spline to be smooth up to its second derivative. A second improvement was to modify the way the random unit vector is assigned to 
a grid point to prevent axis-aligned clumping of noise values.

Both Perlin noise and its improved variant are very fast, simple, and produce noise which is roughly bandlimited. For these reasons, techniques based on Perlin noise remain extremely popular for creating procedural texture. However there are several drawbacks not addressed by either variant, and we discuss two of the major problems here. A first problem is that Perlin Noise exhibits lattice artifacts where it is evident the texture had been generated from an underlying grid (lattice in $\mathbb{R}^{3}$ ) structure. To see this, we notice that the value, and its gradient, of Perlin noise at each grid point is zero. A second problem is that it provides only a general approximation to bandlimited white noise. As described by Cook et al. [22], Perlin noise contains frequencies which create both loss of detail and aliasing artifacts.

\subsubsection{Sparse Convolution and Gabor Noise}

In the previous section, we discussed Perlin noise, a well-known lattice-based procedural noise function. One of the major limitations of Perlin noise was that it exhibits lattice artifacts due to noise values being sampled from a underlying grid. We can consider interpolating from a random set of points, rather than a regular lattice. A well-known method for doing so is sparse convolution noise [48].

Sparse convolution noise is implemented by doing the following. First, a set of randomly located variable magnitude impulses are scattered across the domain. The value of the noise function at a point is obtained as the convolution of the impulses with an appropriate low-pass filter. The width of the low pass filter roughly controls the power spectrum of the resulting noise. This approach removes the lattice artifacts of Perlin noise, but because the contribution of each impulse from the kernel must be calculated, it is also much more computationally expensive. Lewis used a smooth cosine kernel as the low-pass filter.

Lagae et al. [43] used the Gabor kernel in a random pulse process to generate noise. Like sparse convolution noise, a set of pulses are distributed across the domain, but the Gabor kernel is used instead of the smooth cosine kernel. The noise is defined as the summation of the randomly distributed Gabor kernels. The choice of the Gabor kernel allows for precise spectral control with controllable bandlimits, and can be used for both anisotropic and isotropic noise generation. The approach of Lagae also has other desirable characteristics. The method is continuous, uses memory independent of the complexity of the texture, and can be evaluated at interactive speeds. 
Lagae later improved upon Gabor noise [44] by introducing an isotropic kernel for Gabor noise, an error analysis of Gabor noise from kernel truncation, and spatially varying Gabor noise. Other researchers have also developed Gabor noise for other contexts. Galerne et al. consider automatically estimating Gabor noise parameters from exemplar textures [31]. Zanni et al. considered Gabor noise for the texturing of implicit surfaces [95].

\subsection{Texture Basis Functions}

\subsubsection{Worley Noise}

Procedural noise functions generate random sequences of numbers over the texture domain, and noise functions are distinguished from one another by the spectral characteristics of the produced noise. Gabor noise provided a precise way of controlling the magnitude, orientation, principal frequency and bandwidth of the output noise.

There are alternative formulations which do not seek to mimic particular spectral characteristics, but are nevertheless useful in generating interesting texture. These texture basis functions [42] are used to directly generate interesting patterns, and are considered as complementary to the procedural noise functions discussed earlier.

A well-known example of a texture basis function is the Worley Noise function [91]. To implement Worley Noise, a set of feature points are distributed across the domain, and the value at a location is determined by some linear combination of the distances from these points.

We denote $F_{1}(p)$ as the distance from $p$ to the nearest feature point, $F_{2}(p)$ as the distance to the second nearest features point, and more generally $F_{n}(p)$ as the distance to the $n$th nearest feature point. The distance function $f(p)$ of the texture is given by

$$
f(p)=\sum_{i} A_{i} F_{i}(p),
$$

where $A_{i}$ is the scalar coefficient for $F_{i}(p)$. Values of the distance function are mapped onto colors to produce a texture. A simple texture can be created by using only the distance to the nearest feature point $F_{1}(p)$. Distance values will increase radially around the a feature point, and mapping this distance onto a color produces a simple Voronoi pattern. 
More interesting patterns can be generated by considering different linear combinations of distances from points, as shown in Figure 2.2. Worley noise has also been generalized by varying the metric used for distance computation [25].

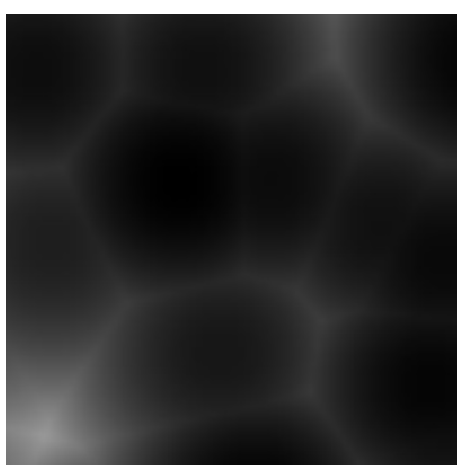

(a) $F_{1}$

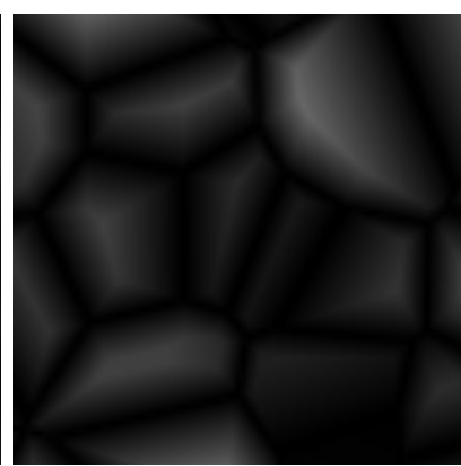

(b) $F_{2}-F_{1}$

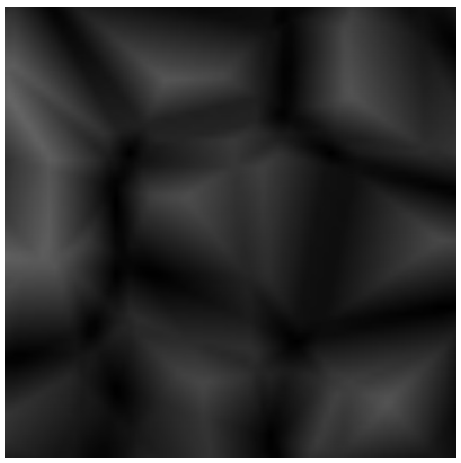

(c) $F_{3}-F_{1}$

Figure 2.2: Examples of Worley noise.

The distance functions $F_{i}(p)$ are in a non-decreasing order, where $0 \leq F_{1}(p) \leq$ $F_{2}(p) \leq F_{n}(p)$, and are everywhere continuous. To give an intuition of the behaviour of Worley noise, we consider only the distance to closest feature point $F_{1}(p)$. If we continuously vary $p$, the function $F_{1}(p)$ remains continuous. However, the gradient of $F_{1}(p)$ will be discontinuous whenever the distance calculation switches from one feature point to another. More specifically, gradient discontinuities exist along line segments which are exactly equidistant from two or more feature points, and for the case of $F_{1}(p)$ are the Voronoi boundaries of the feature point.

Similar gradient discontinuities occur for a general $F_{n}(p)$ as feature points are reordered depending on their nearest distance to $p$. These discontinuities are what give Worley noise their cellular Voronoi-like appearance, but an alternative formulation must be used to create a smooth texture.

\subsubsection{Partition of Unity Textures}

Worley noise produces textures that are continuous, but contain gradient discontinuities. An alternative formulation by Caron and Mould [17], referred to as partition of unity textures, creates an everywhere smooth texture continuous in its first and second derivatives. 
Like sparse convolution noise, partition of unity textures first distributes a set of feature points across the domain, and has each feature point assigned a random value. From this set of points, a triangulation is constructed. Each feature point $x_{i}$ has a set of axes, where each axis is an edge originating at $x_{i}$ and terminating at its neighbor in the triangulation. The value at a location $p$ in the texture is the weighted average of the values from each of the feature points.

The weight of a feature point at a location $p$ is calculated as follows. The location $p$ is projected onto each axis of the feature point. Each projection is then normalized as a fraction of the axis length, and is clamped to be between zero (exactly on the feature point) and one (beyond the axis length). The normalized distance is then transformed with a spline function to have a derivative of zero at both ends. The weight is computed as the product of these transformed distances.

The noise value $f(p)$ at location $p$ is calculated as follows.

$$
f(p)=\frac{\sum_{i} w_{i}(p) v_{i}}{\sum_{i} w_{i}(p)}
$$

The function $w_{i}(p)$ gives the weight of the feature point $x_{i}$, and $v_{i}$ is a random variable associated with $x_{i}$. Partition of unity textures can be considered an interpolation method; the texture smoothly interpolates the feature points where $f(p)=v_{i}$ when $p=x_{i}$.

This method of interpolation was proposed by Runions [70], and Caron [17] used this method for texture. Smooth organic shapes were generated by varying the point distribution, and mapping the resulting values onto colors. The usefulness of this formulation can be seen by considering the region of influence, or support, of the feature point $x_{i}$. The boundary of support of $x_{i}$ can be considered as the intersection of half-planes, where each half-plane is perpendicular to its associated axis and passing through $x_{i}$ 's immediate neighbor. If the Delaunay triangulation is used, this boundary can be considered as an extended Voronoi region; dividing all axes lengths by two exactly reproduces the Voronoi diagram. Figure 2.3(b) illustrates the support of a single point of the texture.

If the underlying triangulation is the Delaunay triangulation, we can loosely describe partition of unity textures as a blending of smooth Voronoi regions. The produced texture would be similar to a texture produced by Worley noise when $F_{1}(p)$ (the distance to the nearest feature point) is used. We show an example texture 

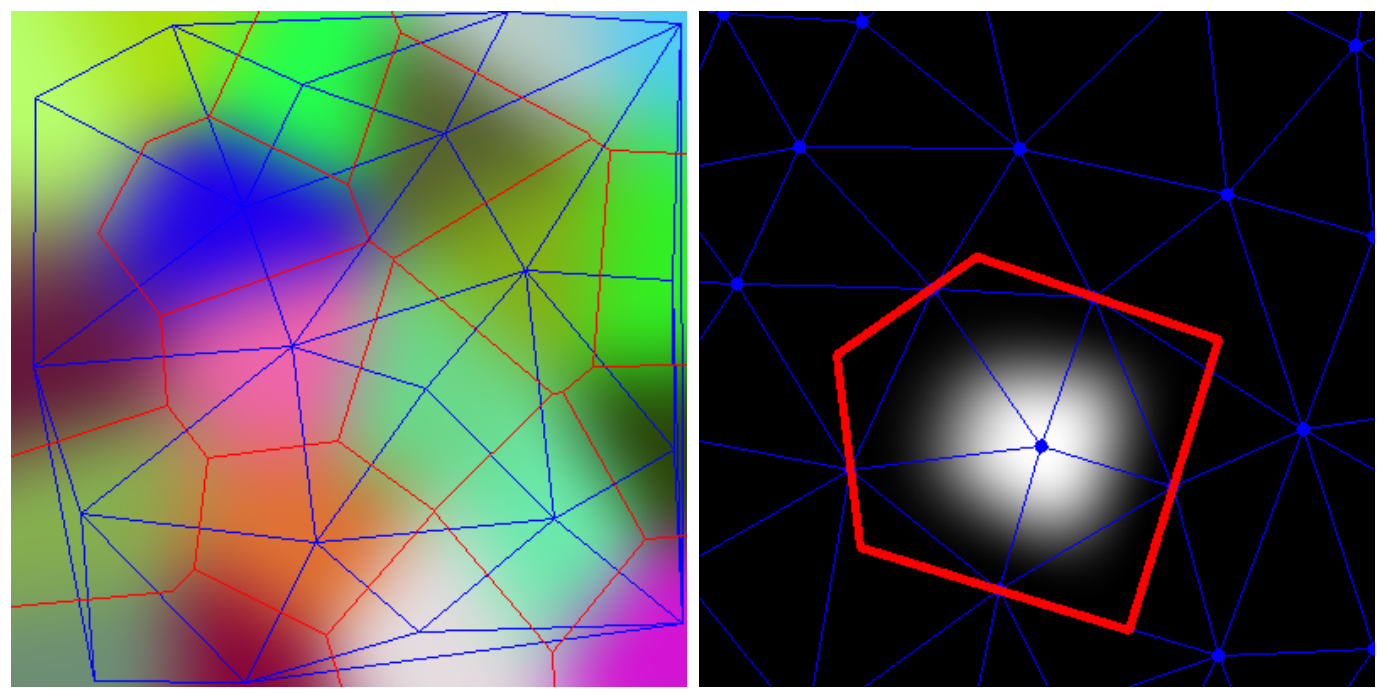

Figure 2.3: Behaviour of partition-of-unity textures. (a) texture created by assigning a random color to each point, and in (b) the support of a feature point is shown as a red outline, and the underlying graph is shown in blue.

created using partition of unity in Figure 2.3(a); the triangulation of the points is shown in blue, and the Voronoi diagram is shown in red on the texture.

\subsection{Reaction Diffusion Textures}

Reaction-diffusion was first described by Alan Turing [83] as a chemical mechanism for pattern formation. Several researchers have since described how certain simple patterns could be generated with such a mechanism $[6,55,58]$. Reaction-diffusion systems generate organic and smooth patterns, and has been made popular for use in procedural texture by the work of Witkin and Kass [90], and Turk [84]. We first give a simple overview of the reaction diffusion method, and we then discuss its extensions to generate more elaborate textures.

The method generates texture by simulating two or more chemicals interacting in a system. Chemical concentrations are mapped to colors to generate the texture. In this model, the chemical concentrations within the system are governed by two separate processes of reaction and diffusion. Diffusion is the process of chemicals moving from a point of higher concentration to a lower concentration, while reaction describes the interactions between chemicals. If we consider a simple two chemical system of chemicals $a$ and $b$, reaction-diffusion can be described with the following 
equations:

$$
\begin{aligned}
& \frac{\partial a}{\partial t}=F(a, b)+K_{a} \nabla^{2} a \\
& \frac{\partial b}{\partial t}=G(a, b)+K_{b} \nabla^{2} b .
\end{aligned}
$$

The first equation describes the change in concentration of chemical $a$ over time at a fixed position. This change is dependent on the function $F(a, b)$ of the local concentrations of $a$ and $b$, and the difference in neighbor concentrations of chemical $a$ scaled by some constant $K_{a}$. The operator $\nabla^{2}$ is the Laplacian operator, and $\nabla^{2} a$ will be positive if chemical $a$ diffuses toward this position and negative if chemical $a$ diffuses away from this position. Similarly, the second equation describes the change in concentrations of chemical $b$.

With reaction-diffusion, only a small initial variation of chemicals is required to drive a complex simulation. The system would converge to a stable state where chemicals are distributed across the domain based on the reaction-diffusion equations. A limitation of using reaction-diffusion is the difficulty in selecting a set of equations to generate a particular texture. Furthermore, only an approximate solution can be obtained through numerical simulation. Turing gave the following simple discrete simulation in one dimension:

$$
\begin{aligned}
\triangle a_{i} & =K_{s}\left(16-a_{i} b_{i}\right)+K_{a}\left(a_{i+1}+a_{i-1}-2 a_{i}\right) \\
\triangle b_{i} & =K_{s}\left(a_{i} b_{i}-b_{i}-\beta_{i}\right)+K_{b}\left(b_{i+1}+b_{i-1}-2 b_{i}\right) .
\end{aligned}
$$

In a single dimension, the simulation produces irregular peaks and valleys in the chemical concentrations of $b$ relative to $a$. In two dimensions, a similar procedure gives spot patterns. A five-chemical system by Meinhardt [55] can be used to create stripe patterns (Figure 2.4). Witkin and Kass proposed several modifications to traditional reaction-diffusion to vary the types of patterns generated [90]. Their work proposed a method of anisotropic diffusion which varied the diffusion rates of chemicals depending on direction. They also described space-varying diffusion where parameters of the system varied across the domain, for use in non-stationary patterns.

Turk [84] used a series of reaction-diffusion systems, described as a cascade process, to create more complex patterns. In this approach an initial system is run, and based on its chemical concentrations, certain regions of the system are marked as fixed. 


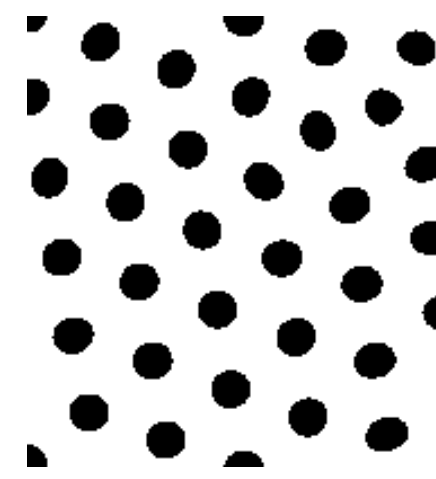

(a)

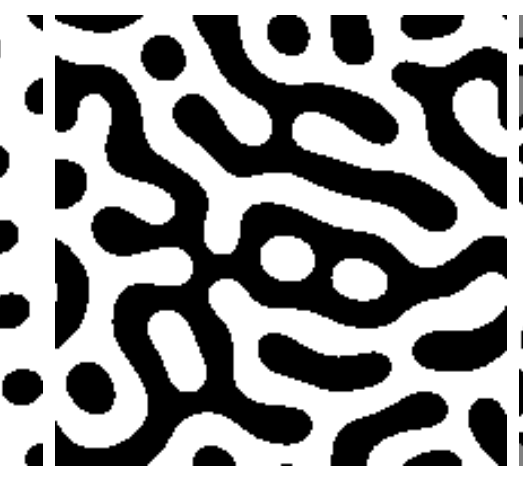

(b)

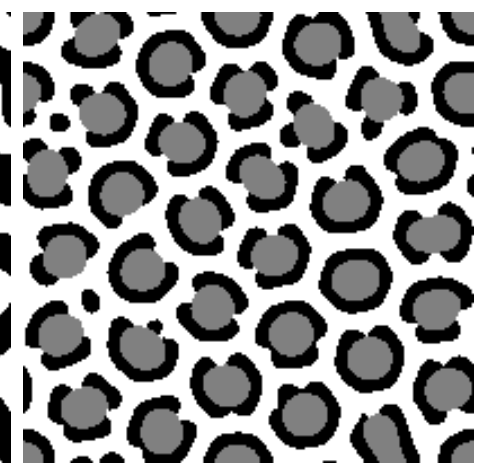

(c)

Figure 2.4: Examples of reaction-diffusion. (a) spots in simple two chemical system, (b) stripes in five chemical system by Meinhardt, and (c) leopard spots created using cascade system.

A different reaction-diffusion system is then run, but chemicals are only allowed to change outside the fixed regions. The idea is that the fixed regions act as a set of constraints for a secondary system. This process of applying constraints on a simulation can be repeated iteratively with varying fixed regions. Leopard spots can easily be created with this method: an initial system lays down large spots in the texture, and a secondary system refines each spot by adding surrounding details (Figure 2.4).

A standard reaction-diffusion system is solved using a discrete simulation on a grid, but it is often desirable for a texture to be rendered on a general surface. Witkin and Kass proposed a method using anisotropic diffusion as a correction mechanism. In this method, the output texture would appear distorted on a grid, but would then appear correctly on a parametric surface. Turk proposed an alternative method that distributed points on the surface of the mesh, and having reaction-diffusion run directly on this surface. This problem of rendering on a surface is a common problem in texturing, and is discussed further in Section 2.6.4.

\subsection{Exemplar-Based Textures}

Exemplar-based methods rely on a sample exemplar image to synthesize a texture. The aim is to produce an arbitrarily large output image visually similar to the sample, but without artifacts or repetition. These methods are not procedural, but are still 
widely used and well-studied.

These methods are usually limited in replicating textures which are based on a local and stationary process. In other words, a pixel from the texture is dependent on a fixed neighbourhood of nearby pixels, and that this neighborhood size is the same everywhere in the image. This is referred to as the Markov random field model for texture [87].

Another limitation is that exemplar-based methods have traditionally been slow, which is a consequence of the output texture needing to search the exemplar to ensure similarity. Furthermore, exemplar-based methods do not typically produce a resolution-independent texture. We consider extensions of traditional exemplar-based methods which address these limitations in Section 2.6.4.

We discuss three major approaches for implementing exemplar-based textures. First, we discuss pixel-based methods, where textures are generated one pixel at a time. We next discuss patch-based methods, where textures are generated by synthesizing groups of pixels at a time, but require additional care to handle when patches overlap. We lastly discuss optimization-based approaches, where the output texture is formulated as an energy minimization problem. We conclude with a discussion on later works which address some of the original limitations of these methods. For a more thorough discussion, the reader should consult the complete survey on exemplarbased textures by Wei et al. [87].

\subsubsection{Pixel-Based}

Pixel-based methods operate by iteratively copying a single pixel from an exemplar image to an output image. A well known pixel-based method is due to the work of Efros and Leung [27], which later became the foundation of many other exemplarbased algorithms. Its approach is simple, but still allows for the generation of many different types of patterns.

The algorithm of Efros and Leung is initialized by first copying a random region from the exemplar image to the output image. This region is then grown outward one pixel at a time through a neighborhood search process. That is, for a user specified neighbourhood (say $5 \times 5$ ) centered at an output pixel, a set of similar neighbourhoods are found in the exemplar image. The output pixel is set to be the center value of an exemplar neighbourhood randomly selected from this set. The neighbourhood size is a parameter of the method and should be set to be the size of the biggest regular 
feature [27].

To measure the similarity between output and exemplar neighbourhoods, each known pixel value in the output is compared with the corresponding pixel in the exemplar. The squared difference between corresponding pixels are taken, and then multiplied with a weight depending on the spatial distance from the output pixel. Efros and Leung used a Gaussian function so that pixels further away from the output pixel are weighted less. The sum of the weighted values give the distance measure.

Because only some of the pixels in an output neighbourhood may be known (because they have not yet been processed by the algorithm), a normalization process is used where only a subset of pixels are used for matching.

The searching process of this method is very expensive because it requires a linear search through all exemplar neighbourhoods, and is due to an output neighbourhood having a varying number of known pixels for matching. A later method by Wei and Levoy [88] uses a fixed size output neighbourhood. This allows techniques such as tree-structured vector quantization [88] or k-coherence [82] to accelerate matching.

\subsubsection{Patch-Based}

Patch-based methods operate by iteratively copying groups of pixels (patches) from an exemplar image to an output image. The motivation for patch-based over pixelbased methods is that there should be an improvement in quality, as pixels that are together in the exemplar should already be correctly related to one another. The major issue is to correctly handle joining seams of copied patches.

The approach taken by Praun et al. [65] is to have patches overlap and simply overwrite previously synthesized pixels. By varying the shape of patches, this approach is simple but works surprisingly very well for stochastic textures [88]. Liang et al. [50] blended between overlapping patches, but this may cause blurry output in some instances.

Instead of attempting to blend overlapping patches, alternative approaches consider cutting patches to fit coherently with each other. Efros and Freeman considered finding an optimal cut of a patch using dynamic programming [26], and this work was later improved using graph cuts by Kwatra et al. [41]. Another approach is to warp patches so that a pattern is continuous across patch boundaries $[79,92]$. 


\subsubsection{Optimization-Based}

Optimization-based methods operate by minimizing some global energy function. The energy function is based on the differences between exemplar and output neighbourhoods, and a lower energy implies a more similar texture. The energy function can be expressed as the following equation:

$$
E(X, Z)=\sum_{p}\left|x_{p}-z_{p}\right|^{2}
$$

In this equation, $X$ is the output image where $\left\{x_{0} x_{1} \ldots x_{n}\right\}$ is a set of overlapping output neighbourhoods, and $Z$ is the exemplar image where $\left\{z_{0} z_{1} \ldots z_{n}\right\}$ is a corresponding set of neighbourhoods in the exemplar which are most similar to each $x_{i}$.

Kwatra et al. [41] used an Expectation-Maximization (EM) like algorithm to minimize $E(X, Z)$. In the expectation step, the set of similar neighbourhoods $\left\{z_{0} z_{1} \ldots z_{n}\right\}$ remain fixed and the output texture $X$ is solved through a least squares method. In the maximization step, the image $X$ is fixed and the set of similar neighbourhoods are recomputed using tree search. The method alternates between these two steps until convergence, or until a fixed number of iterations have occurred.

The method by Kwatra has several drawbacks. The first issue is that neighbourhood search for each $z_{i}$ is computationally expensive; each search is $O(\log N)$ where $N$ is the number of neighbourhoods in the exemplar. The second issue is that blurring occurs where output neighbourhoods overlap. These drawbacks were later addressed by Han et al. [35] through the use of a k-coherence algorithm in both the expectation and maximization steps.

\subsubsection{Surface Texture Synthesis}

The exemplar-based methods we have described assume that the output is generated on a discretized grid, but it is often desirable to render to a general surface. A parametric mapping can be done between the discretized grid and the surface, but this would introduce undesirable distortion and texture seams. We instead consider methods which generate output texture specific to a particular surface. We describe the work of Turk, but other methods such as those by Ying [94] or Soler [79] can also be used. 
Turk first distributes a set of points regularly along the mesh surface. This is done by randomly placing points on each triangle in the mesh, and using a point relaxation scheme where neighbouring points repel each other. Once points are evenly distributed, a newly formed mesh is created from this point set. This secondary mesh is created to ensure points are evenly spaced for texturing.

After creating the secondary mesh, a vector field is defined on the mesh surface, and is used to control the orientation of the texture. The field is defined by having a user specify directions at several locations on the mesh, and using a diffusion method to propagate the field everywhere on the surface. A second vector field is then created by rotating this field $90^{\circ}$. Taken together, these two fields are used to define a local coordinate system.

Once a local coordinate system is defined everywhere along the surface, texture synthesis can proceed similar to the pixel and patch methods described earlier. Neighbourhoods are defined along a local coordinate system, instead of the canonical Euclidean axes. Turk used this method in a hierarchical manner to improve performance and texture quality.

\subsubsection{Extensions}

Traditional exemplar-based methods are costly in several ways. The methods we have described require the entire output image to be generated at once, which implies a noncompact representation. This also impacts performance because often only a subset of a texture needs to be used for rendering. These limitations lead to the development of texture-on-the-fly methods which provide random access of synthesized texture. Later methods by Wei and Levoy [89] and Lefebvre and Hoppe [47] support random access synthesis of texture.

Exemplar-based methods are also usually done at particular sampled resolutions where a separate interpolation process is done during rendering. Work by Wang et al. [86] considered directly synthesizing a vector representation of an exemplar texture. This was done by using signed distance function to define region boundaries, and colors in regions were represented using compact radial basis function.

The methods we described so far have only generated stationary patterns, but other methods can generate globally-varying textures. This can be done with the introduction of a control map, which describes the global behaviour of the texture. The work of Ashikhmin [4] synthesized non-stationary textures by allowing a user to 
provide a rough color map.

\subsection{Implicit Modeling Methods}

Instead of using automatic methods to generate texture, detail on surfaces can be generated directly using traditional modeling techniques. Implicit modeling techniques are particularly useful because they provide a resolution-independent representation where blending is defined in a natural way.

An implicit function $f(x, y)$ in $\mathbb{R}^{2}$ can be described as the composition of a distance function $d(x, y)$ with a fall-off filter function $g(r)$ where $r$ is the distance to some primitive.

$$
f(x, y)=g \circ d(x, y)
$$

As an example, let us consider a point primitive with fall-off filter $g(r)=1 / r^{2}$. The field is maximal at the point, and rapidly diminishes as the distance from the point increases. If we place several points randomly in the plane and sum their field functions, we can create a texture by mapping the field value to a color.

General splatting of point primitives is an early method of generating texture, and a thorough discussion is given by Schachter [72]. Partition of unity textures can be considered as splatting point primitives over the domain with asymmetric field functions.

We can generalize splatting to include other types of primitives. Other primitives such as line-segments, triangles or curves can be used, so long as distance to the primitive is well defined.

We can also generalize how fields are composed. A simple example is to take the minimum or maximum of the fields. Ricci [69] proposed summing fields exponentiated to the $n$th power and then taking the $n$th root. Another method combines fields of primitives in a hierarchical manner by combining two primitive fields at a time [93]. To obtain more complex texture, an artist can specify the exact implicit primitives and blending relations to use.

The subject of implicit modeling covers many techniques, and include skeletal implicit modeling, offset surfaces, level sets, variational surfaces, and algebraic surfaces [77]. Implicit modeling is usually discussed in the context of surface modeling, where the zero set of the implicit field defines the boundaries of a three-dimensional model surface. For example, Galbraith et al. [30] previously used implicit modeling 
techniques to model the Murex cabritii sea shell. Several examples using implicit modeling are described by Gomes [32]. These examples show that implicit methods are able to generate very complex surfaces, but require detail to be explicitly specified.

The traditional strength of implicit surfaces is that they naturally define the entire interior of a surface; field values greater than some threshold are said to belong to the surface. Point classification (determining whether a point is inside, outside or on the boundary of a surface) is also straightforward. Bloomenthal et al. [14] gives a thorough discussion of implicit modeling in comparison with alternative parametric representations.

Using similar approaches to Galbraith et al. and Gomes, complex texture can be created. Instead of using the zero set of a field as a boundary surface, field values of the primitives are mapped to colors. Smooth color transitions are obtained by blending of field values. A key difference is that the whole implicit field, not only the zero set, must be well behaved. This concern is revisited for smooth distance fields in Chapter 3.

Our approach, described next, uses many of the techniques found in implicit modeling. We generate complex texture, but without explicit specification of primitives.

\subsection{Smooth Distance Field Textures}

We described several methods for procedurally generating texture. Noise-based functions, such as Perlin Noise and Gabor Noise, produce random patterns approximately bandlimited to a range of frequencies. Worley noise and partition of unity textures generate Voronoi-like cellular patterns using distances from randomly distributed points. Reaction-diffusion methods generate organic and smooth patterns through a simulation of chemical processes.

We also described exemplar-based methods for generating texture. These methods are not procedural, but use a user supplied exemplar image. These methods can generate a large variety of texture, but usually require a discretization of the surface, and are typically slower than procedural approaches.

Lastly, we have considered modeling texture directly using implicit surfaces. Implicit surfaces provide a resolution independent representation, and provide a natural method for combining primitives. These methods have been used successfully to model organic surfaces, but require an artist to explicitly specify geometry. Implicit 
surfaces can be used for texture synthesis by mapping field values to colors.

Our method combines the advantages of several approaches, we are able to generate smooth, continuous and organic patterns. Like partition of unity textures, our method is parameterized only by a set of labeled points on a mesh, but is capable of generating more general curvilinear shapes. Our method is procedural, smooth, and does not require a grid discretization.

The solution we provide relies on the blending of smooth normalized distance fields between primitives, and is similar to the previously described implicit modeling methods. We review existing methods for creating smooth distance fields in the next section. 


\section{Chapter 3}

\section{Smooth Signed Distance Functions}

\subsection{Definition}

Computing the distance from an object is a fundamental operation in implicit modeling. Section 2.7 described how an implicit field is constructed by composing a distance function with a fall-off filter function, with the boundary of a surface defined as some isocontour of the created field. For some applications, including our method for creating texture, distance fields are required to be smooth and everywhere differentiable. This chapter reviews different methods for defining a smooth signed distance function to create such a field.

A smooth signed distance function can be described as a smooth approximation of the exact signed distance function. A formal definition of the exact signed distance function $f(p, \Omega)$ of a region $\Omega$ can be expressed by the following equation:

$$
f(p, \Omega)=\operatorname{sign}(p, \Omega) \cdot \operatorname{dist}(p, \partial \Omega)
$$

where $p$ is a point in the domain, $\Omega$ is the defined region, and $\partial \Omega$ is the boundary of that region. The function $\operatorname{dist}(p, \partial \Omega)$ is the Euclidean distance to the region and is given by

$$
\operatorname{dist}(p, \partial \Omega)=\inf _{x \in \partial \Omega}\|x-p\|
$$


The function $\operatorname{sign}(p, \Omega)$ is the region's sign function, and is given by

$$
\operatorname{sign}(p, \Omega)= \begin{cases}-1 & p \epsilon \Omega \\ 0 & p \epsilon \partial \Omega \\ +1 & \text { otherwise. }\end{cases}
$$

That is, the sign of the distance function is negative in the interior of a region, zero at the boundary, and positive otherwise. The zero set of a distance field is the set of points that lie on the boundary of the surface. The exact signed distance function is defined everywhere in the domain for one or more regions.

A limitation of using an exact signed distance function is that the produced field may contain gradient discontinuities, even if the boundary of the surface is smooth. For example, if the boundary is a circle, there exists a single point of gradient discontinuity at the circle's center. If the boundary is an ellipse, this gradient discontinuity stretches into a line.

More generally, gradient discontinuities exist along the medial axis of the region. The medial axis is where a point is equidistant to two or more points of the region boundary. Described in terms of the smoothness of a function, an exact distance function is $C^{0}$ continuous everywhere, but has $C^{1}$ discontinuities at the medial axis of its associated region. For any location where the gradient is well-defined, the gradient magnitude is unity, and the gradient orientation is in the same direction as the normal at the nearest point of the region boundary.

A smooth signed distance function is an approximation of the exact distance function that is smooth everywhere, except possibly at the region boundaries. There are several methods for constructing smooth signed distance functions, and different methods are used depending on the application. For our purposes, we desire distance functions that are continuous and fast to evaluate. We also desire our distance function to be well-behaved, in the sense that the field does not contain widely varying values some fixed distance away from the region boundary. In the next section, we will describe how to characterize a signed distance function by its behaviour at the zero set. 


\subsection{Normalized Approximation}

The work of Biswas and Shapiro [11] characterized a smooth distance function by its derivative values at the zero set. Recall that for an exact Euclidean distance function, for every point where the gradient is well defined, the magnitude of the gradient is unity and all higher derivatives in the direction of the gradient vanish. For a smooth distance function, the gradient may be unity at the zero set, but there must be some non-zero higher derivatives to create a smoothed field.

Specifically, let $w$ be a smooth approximation to an exact distance function, and let $\bar{v}$ be the vector of the gradient field at the zero set. As described by Shapiro [75], the smooth distance function $w$ is said to be normalized to the $m$ th order if higher order derivatives of degree up to $m$ vanish. In other words, if $w$ is normalized to order $m$, then

$$
\frac{\partial w}{\partial \bar{v}}=1 ; \frac{\partial^{k} w}{\partial \bar{v}^{k}}=0 ; k=2,3, \ldots, m .
$$

We can describe how well a smooth distance field approximates a corresponding exact distance field by considering the degree of normalization. The degree of normalization is, however, a limited description of the behaviour of the function; normalization is a local property of the zero set and does not describe behaviour away from the boundary. For our method, we desire distance fields to have a gradient of unity at the boundary, but also to be well-behaved away from that boundary.

In the remainder of this chapter, we will discuss different methods for constructing smooth signed distance fields. We will describe different interpolation, potential, discrete, and constructive methods, and we frame our discussion on whether a method can produce a continuous, smooth, and well-behaved distance field.

\subsection{Interpolation-Based Methods}

Interpolation-based methods construct a smooth distance field by sampling an exact distance field at a set of points, and then smoothly interpolating that point set.

Interpolation of distance values from points is closely related to the scattered data interpolation problem [2]. Given a set of unstructured value-location pairs, scattered data interpolation obtains a function over the entire domain which interpolates the given data points.

More formally, given a set of $\mathrm{N}$-dimensional data points $\left\{x_{1} x_{2} \ldots x_{n}\right\}$ associated 
with scalar data values $\left\{v_{1} v_{2} \ldots v_{n}\right\}$, scattered data interpolation obtains a function $f(p): \mathbb{R}^{N} \rightarrow \mathbb{R}$ for all $p$ such that $f\left(x_{i}\right)=v_{i}$ for $i=1 \ldots n$.

Note there can be infinitely many such solutions for this interpolation so long as the function passes through the prescribed data points, and therefore the quality of the interpolation depends on the application. There are many scattered data interpolation techniques, but we focus on the well-known inverse-distance, naturalneighbour, and radial basis interpolation methods. Because our focus is on generating texture, we restrict ourselves to interpolating over the image plane.

\subsubsection{Inverse-Distance Interpolation}

A well-known and simple method of scattered data interpolation is Shepard's method [76], where the interpolant is defined by

$$
f(p)=\left\{\begin{array}{ll}
\sum_{k} \frac{\left\|p-x_{k}\right\|^{M}}{\sum_{j}\left\|p-x_{j}\right\|^{M}} v_{k} & \text { if }\left\|p-x_{i}\right\| \neq 0 \text { for all } i \\
v_{i} & \text { if }\left\|p-x_{i}\right\|=0 \text { for some } i
\end{array},\right.
$$

where $v_{i}$ is the value associated with data point $x_{i},\left\|p-x_{i}\right\|$ denotes the Euclidean distance between the query point $x$ and the supplied data point $x_{i}$, and $M$ is a positive constant.

This method is simple and extends to arbitrary dimensions, but has several limitations as mentioned by Shepard. A major drawback of this method is that it is a global interpolant; that is, the interpolated value depends on all data points. A global interpolation has performance implications because the interpolation complexity becomes linear in the number of data points. A modified Shepard's method was later proposed by Renka [68], and interpolates using only a local set of data points. In the method by Renka, each data point contributes to the interpolation calculation only within a given radius of the datapoint. Both methods can be described within a framework for interpolation, which we next describe.

\subsubsection{Interpolation Framework}

Shepard's method, as well as its related methods, can be described in a more general partition of unity framework for interpolation [2]. In this framework, an interpolated 
value is obtained as a weighted combination of data points given by

$$
f(p)=\sum_{i} w_{i}(p) v_{i},
$$

where $w_{i}$ is a non-negative function which weights the contribution of its associated data point $v_{i}$. In addition, the sum of the weights must be equal to unity for any point in the domain, and this requirement can be expressed as

$$
\sum_{k} w_{k}(p)=1 .
$$

The set of weights are said to form a partition of unity. Shepard's method is a special case of this framework where the weight functions are

$$
w_{i}(p)=\frac{\left\|p-x_{i}\right\|^{M}}{\sum_{j}\left\|p-x_{j}\right\|^{M}} .
$$

Different methods of interpolation are obtained by using different weight functions. Another well-known interpolation method is natural neighbor interpolation [78], which relies on the computation of a Voronoi tessellation. The weight for a datapoint is calculated by finding how much of the data point's Voronoi area is taken away when a query point is added to the Voronoi tessellation. Unlike Shepard's method, natural neighbour interpolation provides a local interpolation without needing to specify a radius for each data point. Natural neighbor interpolation is useful when data points are unevenly spaced [46].

In partition of unity textures, the weight function of each data point was obtained as the product of axes (Section 2.4.2), and is another example of an interpolation method. The procedure of interpolating using mesh axes was first described by Runions and Samavati [70], and was considered in the context of generalizing spline curves and surfaces. The fields of each data point in partition of unity interpolation produces smooth and rounded shapes, which Caron [17] used to produce organic texture by interpolating between color values. 


\subsubsection{Radial Basis Functions}

Several interpolation methods, including inverse-distance and natural neighbor interpolation, use weight functions with the Kronecker delta property:

$$
w_{i}\left(v_{j}\right)=\left\{\begin{array}{ll}
1 & i=j \\
0 & \text { otherwise }
\end{array} .\right.
$$

This property provides exact interpolation at the data point, but also results in the interpolation function having gradient zero at that point and appearing flat. Radial basis interpolation is an alternative method of interpolation that does not appear flat at the sampled data points.

In radial basis interpolation [15], a function $\psi(r)$ is selected as a basis to describe a surface. Every data point is associated with a basis function, and the surface is described as the sum of these basis functions over all points. The basis functions are smooth and radially symmetric. Following the earlier notation, let $x_{i}$ be the data point with value $v_{i}$ and let $\psi(r)=\exp \left(-r^{2} / k^{2}\right)$ be the basis function where $k$ is a constant. With the constraint that the function must interpolate the data points, the interpolation function is given by

$$
f(p)=\sum_{i} c_{i} \psi\left(\left\|p-x_{i}\right\|\right) \text { where } f\left(x_{i}\right)=v_{i}
$$

The set of constraints $f\left(x_{i}\right)=v_{i}$ can be written in matrix form as $\mathbf{A c}=\mathbf{b}$ where

$$
\begin{aligned}
& A=\left[\begin{array}{ccc}
\psi\left(\left\|x_{1}-x_{1}\right\|\right) & \cdots & \psi\left(\left\|x_{1}-x_{n}\right\|\right) \\
\vdots & \ddots & \vdots \\
\psi\left(\left\|x_{n}-x_{1}\right\|\right) & \cdots & \psi\left(\left\|x_{n}-x_{n}\right\|\right)
\end{array}\right] \\
& c=\left[\begin{array}{ccc}
c_{1} & \ldots & c_{n}
\end{array}\right]^{\top} \\
& v=\left[\begin{array}{lll}
v_{1} & \ldots & v_{n}
\end{array}\right]^{\top} .
\end{aligned}
$$

Solving the above system gives the particular radial basis interpolation.

The choice of using the Gaussian function ensures that this system is positive definite, and therefore has a unique solution. The Gaussian radial basis interpolation 
we described produces a smooth surface which interpolates the control points, and can reproduce any surface that can be represented as scaled and shifted Gaussians. Simple surfaces, such as a plane, cannot be represented in this manner. However, radial basis interpolation can be extended so that it is possible to reproduce an underlying surface of fixed polynomial degree [37].

Other kernels besides the Gaussian kernel can be considered. Thin plate splines have the property of minimizing the bending energy of an interpolated surface, and are defined using the radial basis kernel $\psi(r)=r^{2} \log (r)$.

Radial basis functions are a very common data interpolation technique, but have also been used for implicit modeling. Variational implicit surfaces [85] use radial basis functions to define the surface boundaries in implicit objects. In a variational implicit surface, values assigned to data points are used as constraints to an implicit model. Positive values assigned to data points are used as constraints for the interior of the surface, zero value data points are used for the boundary of the surface, and negative values are used to define the exterior of the surface. Normal constraints are defined using a zero value and a positive value data point. A three dimensional interpolation of all data points defines the surface. Variational implicit surfaces have been used in many sketch-based algorithms [3,38] to define a three-dimensional model.

A major limitation of using radial basis functions is that determining the weights for interpolation requires solving a dense matrix, which would be computationally expensive for a large number of data points. A faster alternative is to use compactlysupported radial basis functions, where only a sparse matrix needs to be solved [57]. Other approaches divide the points into separate groups and solve each group separately, referred to as partition of unity techniques [59].

Depending on the choice of kernel, another limitation is that radial basis functions are not well-behaved; field values can take on a wide range of values away from the interpolated data points. This behaviour is undesirable for applications that use the magnitude, instead of only the sign, of the distance field. Our method for creating texture does not use radial basis functions because we uses the signed magnitude of distance values to blend between texture elements.

In implicit surface modeling, if an insufficient number of constraints are specified, the variations in field behaviour may create unintended, spurious surfaces. The creation of spurious surfaces from variational implicit surfaces was discussed in detail by Mann [52]. Their work found that using normal constraints produced reasonably 
smooth implicit surfaces, but still found instances of spurious surfaces being unexpectedly created.

A common concern of all interpolation-based methods is that the accuracy and smoothness of the distance field will depend on the sampling density of the data points. The next method we discuss does not create a distance field from sampled points, but instead derives the distance field from the boundary of the associated surface.

\subsection{Potential-Based Methods}

Potential-based methods [11] treat the region boundary as a charged surface. In this model, the field is maximal at the boundary and dissipates away from the surface. A single point charge with an inverse distance kernel would contribute $1 / r^{N}$ to the field at a query point, where $r$ is the distance from the query point to the point charge, and $N$ is some constant. The total field value at any point $p$ is given by the line integral

$$
f(p)=\int_{S} \frac{1}{r(p)^{N}} d S,
$$

where $S$ is the boundary of the surface. This function produces a value of infinity at the boundary, and the field value dissipates further away. We can use the reciprocal of this function as a general distance field. A potential-based distance field has been previously used to quickly compute an approximate medial axis [1].

An alternative potential-based method uses a double-layer potential model. In this alternative model, the boundary of a surface is defined as having a layer of positive point charges on one side, and an opposing layer of negative point charges on the other. For the particular case of $N=1$, the potential value corresponds to the normalization function used in transfinite mean-value interpolation [8]. Smooth signed distance fields using double-layered potentials were later generalized by Belyaev et al. [8].

Potential field methods are attractive for several reasons. Field values can be solved analytically for simple boundaries, such as line segments or circular arcs. Furthermore, because potentials are defined as an integral over a point set, the field does not suffer from the bulging [12] common in many implicit modeling techniques. However, these fields are flat on the boundary (the derivatives of $S$ vanish) and cannot be 
normalized. Furthermore, numerical instabilities occur because field potentials approach infinity towards the zero set. Despite these limitations, further investigation by Belyaev [8] suggests that there is promise in applying potential-based methods for implicit modeling.

\subsection{Discrete Methods}

Previously described methods provided an analytic solution for the field value everywhere in $\mathbb{R}^{2}$. Higher quality smooth distance fields can be obtained at a cost of discretizing the domain onto a fixed grid.

Discrete methods can be used to obtain a smooth distance field. A fast and simple method is to use convolution of the exact signed distance field. To create a smooth distance field using convolution, the exact signed distance field is computed at a particular resolution, say $n \times m$, on a fixed grid. This is done by rasterizing the region boundary onto the grid, and then computing the distance transform. The distance transform will give the Euclidean distance to the nearest boundary pixel for every pixel in the image. We can compute the distance transform in $O(n m)$ time using the approach by Felzenszwalb [29]. The resulting distance field is then smoothed using a convolution given by

$$
g(x)=(w * f)(x)=\int_{\mathbb{R}^{2}} w(x-p) f(p) d p,
$$

where $f(x)$ is the original exact distance field, $p$ is a displacement vector from $x$, and $w$ is a smooth kernel such as a Gaussian. The smoothness of the distance field is parameterized by the choice and width of the kernel. A notable drawback of this approach is that a general convolution does not preserve the zero set of the distance field.

Preservation of the zero set can be done with a variable radius convolution where the width of the kernel decreases based on the distance to the zero set. Sanchez et al. [71] provided a method of variable radius convolution of a signed distance field, given by

$$
g(x)=\int_{\mathbb{R}^{2}} f(x-p h(x)) w(p) d p,
$$

where $f(x)$ is the exact distance field, $p$ is a displacement vector from $x$, and $w$ is a smooth kernel. The function $h(x)$ is used to control the kernel size, and smoothly 
varies from 0 to 1 away from the zero set. One way to define $h(x)$ is to use the smoothstep function such that

$$
\begin{aligned}
& h(x)=3 r(x)^{2}-2 r(x)^{3} \\
& r(x)=\min \left(|f(x)| / f_{c}, 1\right)
\end{aligned}
$$

where $f_{c}$ is some constant. More sophisticated methods of constructing smooth discrete distance fields rely on the solution of particular partial differential equations. Crane considered the use of heat flow to compute the signed distance to a mesh [23]. Belyaev et al. considered several variations of computing smooth distance fields based on the Poisson, screened Poisson, and p-Poisson equations [9]. Although we desire a continuous representation of a smooth signed distance field, high quality discrete distance fields can be obtained using the aforementioned approaches.

\subsection{Constructive Methods}

The approach that we use follows from the theory of R-functions [74], which is a method of constructing implicit functions for given geometric objects. An R-function can be informally defined as a function whose sign (as positive or negative) is completely determined by the sign of its arguments. A change of sign of an R-function can only happen if a change of sign occurs in one or more of its arguments. A simple example of an R-function is the function

$$
f(x, y)=x+y+\sqrt{x^{2}+y^{2}} .
$$

We see that the function is negative only if both arguments are negative. If we treat the sign of the function as a binary variable, this R-function would correspond to logical disjunction $(x \vee y)$. In general, R-functions allow applying logical operators (such as conjunction, disjunction and negation) onto the set of real numbers. Complex shapes can then be composed by applying R-functions onto a set of simple primitives.

We now describe the method by Biswas and Shapiro [11] to construct a smooth distance field using R-functions. The method assumes that the region boundary is composed of a set of connected line segments.

A smooth and continuous distance field is formed by creating an implicit field from each line segment of the boundary, and then applying a separate R-function 
operation to join the implicit fields together. The implicit field for a single line segment is created using the R-function intersection between the implicit fields of an infinite line and a circular disk.

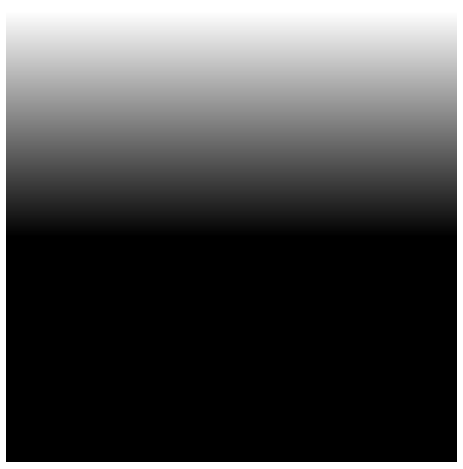

(a)

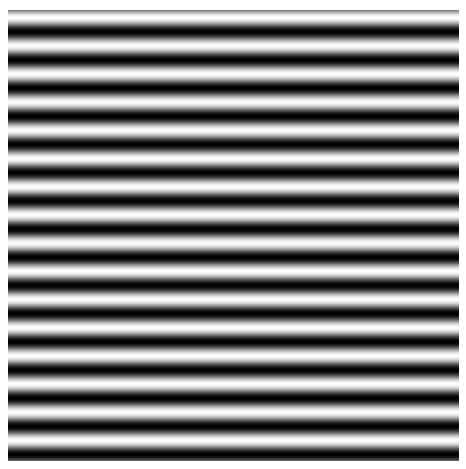

(d)

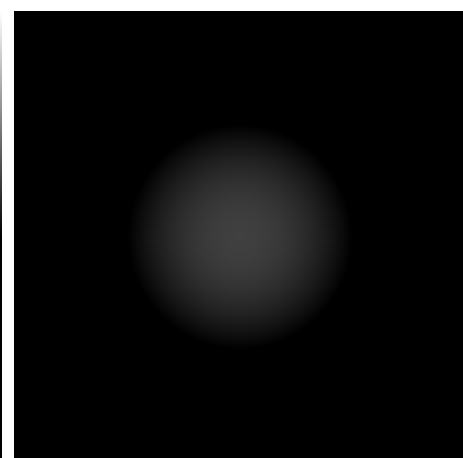

(b)

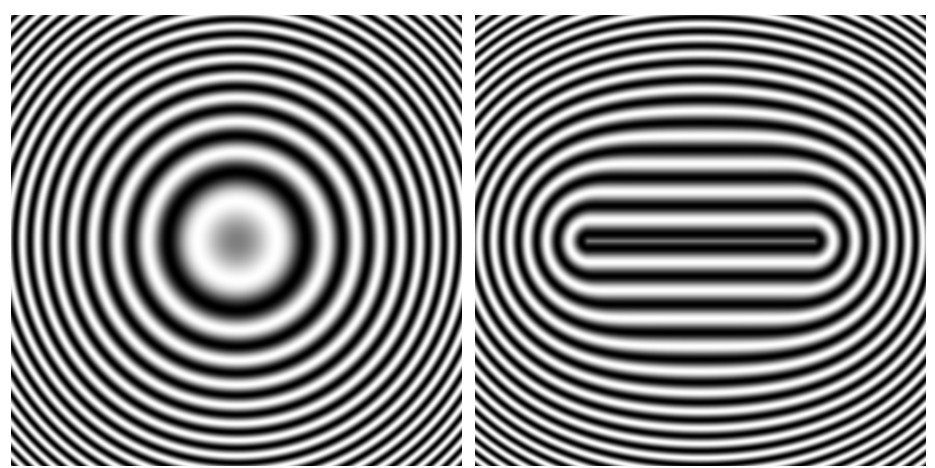

(e)

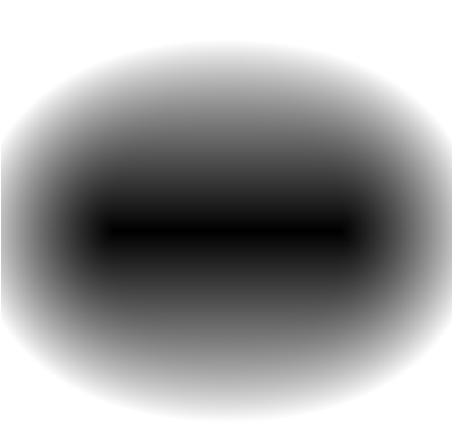

(c)

Figure 3.1: A signed distance field of an infinite line (a) is intersected with the signed distance field of a circular field (b) producing a smoothed field (c). Isocontours of the produced fields are shown on the bottom row.

The implicit field for a single line segment is constructed as follows. For a line segment $\ell=\left(\left(x_{1}, y_{1}\right),\left(x_{2}, y_{2}\right)\right)$, with length $d=\sqrt{\left(x_{2}-x_{1}\right)^{2}+\left(y_{2}-y_{1}\right)^{2}}$, we construct the scalar field

$$
f(x, y)=\frac{1}{d}\left(\left(x-x_{1}\right)\left(y_{2}-y_{1}\right)-\left(y-y_{1}\right)\left(x_{2}-x_{1}\right)\right)
$$

The field $f(x, y)$ is the signed distance function for an infinite line passing through the endpoints of $\ell$, and has a unit gradient everywhere. We next define the circular 
implicit field

$$
t(x, y)=\frac{1}{d}\left[\left(\frac{d}{2}\right)^{2}-\left(x-\frac{x_{1}+x_{2}}{2}\right)^{2}-\left(y-\frac{y_{1}+y_{2}}{2}\right)^{2}\right] .
$$

The field $t(x, y)$ is centered at the midpoint of $\ell$, and has its zero set passing through $\ell$ 's endpoints. The circular field can be derived from the general equation of a circle $\left(x-x_{0}\right)^{2}+\left(y-y_{0}\right)^{2}=r^{2}$. The factor $1 / d$ is used so that the boundary is normalized to the first order.

We intersect the circular field $t(x, y)$ with the infinite line field $f(x, y)$ to produce the implicit field for the line segment. Shapiro described a method to intersect a field $w_{1}$ with another field $w_{2}$ to give a combined field $w$ that is normalized to the first order, given by

$$
w(x, y)=\sqrt{w_{1}(x, y)^{2}+\frac{\left(\left|w_{2}(x, y)\right|-w_{2}(x, y)\right)^{2}}{4}} .
$$

Making the substitution $w_{1}=f$ and $w_{2}=t$ gives the normalized field function for the line segment $h$, and we have

$$
h(x, y)=\sqrt{f(x, y)^{2}+\frac{(|t(x, y)|-t(x, y))^{2}}{4}} .
$$

We show the procedure of creating the implicit field for a line segment in Figure 3.1.

For every line segment of the boundary, we apply the above procedure. Let $L=$ $\left\{h_{1} h_{2} \ldots h_{n}\right\}$ be the field values obtained for the set of boundary line segments. To join the implicit fields of the line segments together, we use a generalized R-equivalence operation. The R-equivalence operation of order $m$ is defined as

$$
d\left(h_{1}, \ldots, h_{n}\right):=\frac{1}{\sqrt[m]{\frac{1}{h_{1}^{m}}+\frac{1}{h_{2}^{m}}+\ldots+\frac{1}{h_{n}^{m}}}} .
$$

The result of applying the R-equivalence operation is an unsigned distance field. To obtain a signed distance function, a point-containment test must be used on the original region to determine the sign of the field. The sign is negative in the interior of a region, zero at the boundary, and positive everywhere else. An example of a smooth distance field of a polygon is shown in Figure 3.2. 


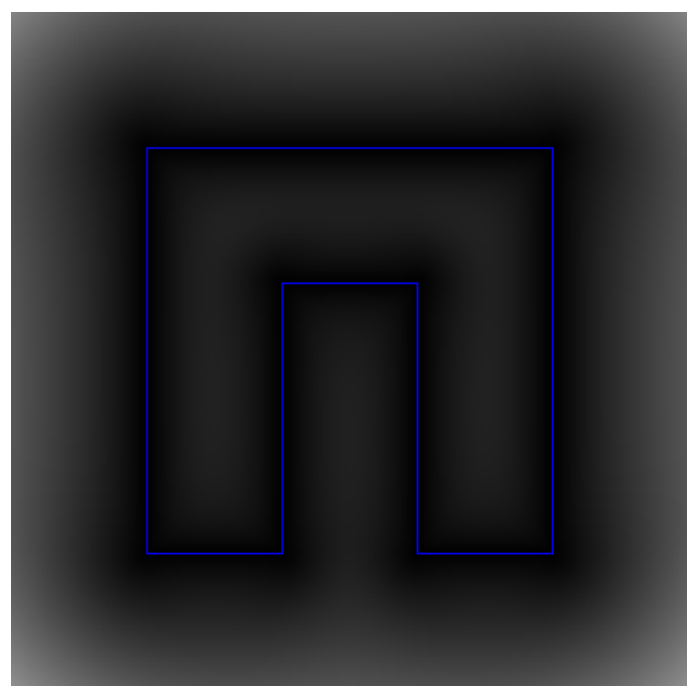

(a)

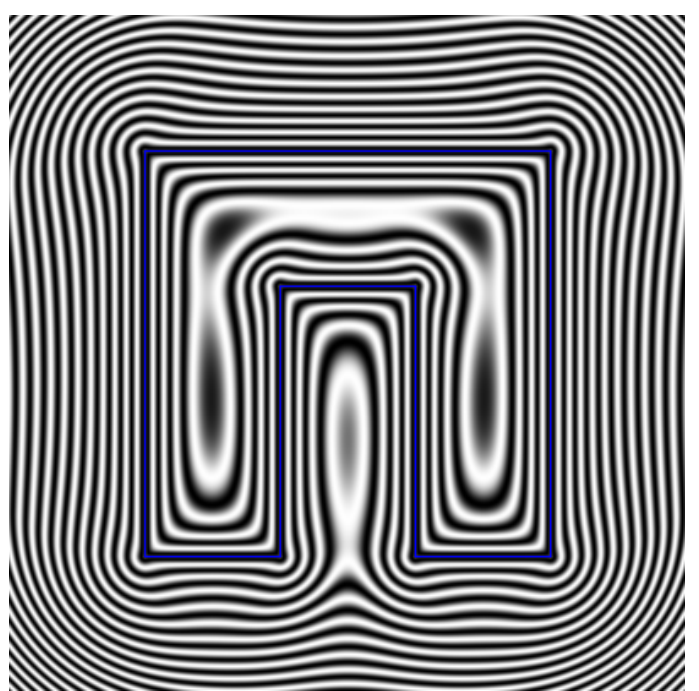

(b)

Figure 3.2: Joining implicit fields. (a) The smooth distance field of a polygon, and (b) isocontours of the produced field.

The distance function of Figure 3.2 is reasonably well-behaved, although the gradient magnitude varies away from the zero set. In the next section, we will use smooth signed distance functions created from R-functions to generate texture. 


\section{Chapter 4}

\section{Smooth Signed Distance Field Textures}

Chapter 2 described several different procedural methods for creating texture. In particular, we described Worley noise and partition of unity textures as methods for creating different cellular patterns.

Worley noise operates by taking linear combinations of distances from points, and different textures are obtained by changing how point distances are weighted in the texture function. Textures produced using Worley noise are everywhere continuous, but have gradient discontinuities wherever the distance calculation switches from using one feature point to another. Partition of unity textures is another method to create cellular patterns. A texture is created by blending a set of convex fields based on the points of a mesh. Unlike Worley noise, textures produced with partition of unity are everywhere smooth.

Worley noise and partition of unity textures both use a set of points to create texture. Different textures are created by either modifying the point distribution, or by changing a small set of input parameters. While many different kinds of textures can be created, neither Worley noise nor partition of unity textures can create smooth non-convex shapes, such as those found in certain fish and lizards. Reaction-diffusion textures are capable of generating various spot and stripe patterns, but require discrete simulations.

In this chapter, we present our procedural method for creating organic patterns. Like partition of unity textures, our method uses a mesh to specify the texture. Instead of defining an implicit field for every point, we use the edges of a mesh to define implicit fields based on distances to line segments. Unlike previous methods, we are able to generate smooth, non-convex shapes in an output texture. Our method has a continuous and compact representation. 


\subsection{Algorithm}

Our method can be broken down into six steps: mesh generation, label assignment, point grouping, contour definition, field creation, and region merging. Each step of our method is shown in Figure 4.1.

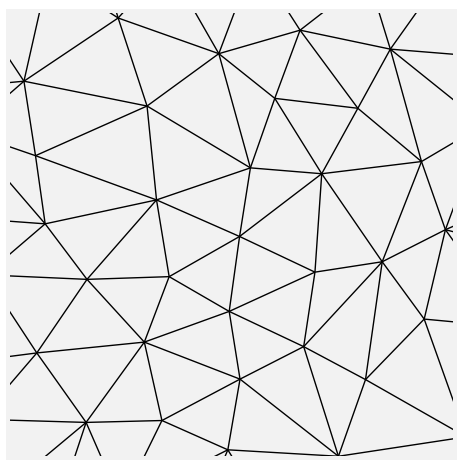

(a) Mesh Generation

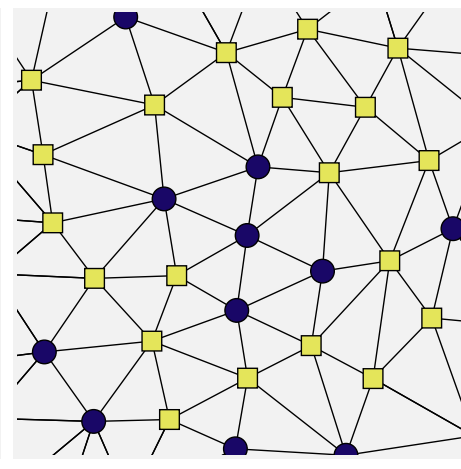

(b) Label Assignment

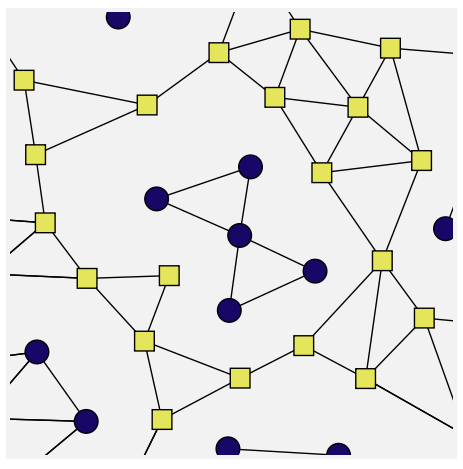

(c) Point Grouping

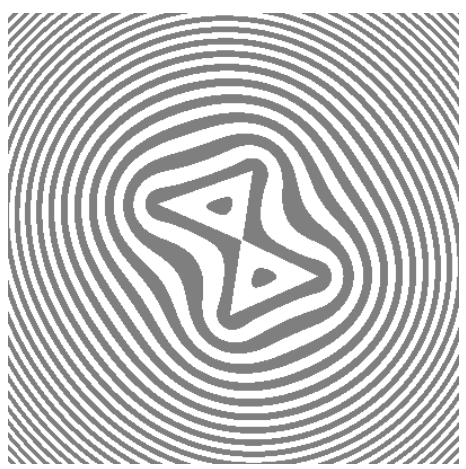

(d) Contour Definition

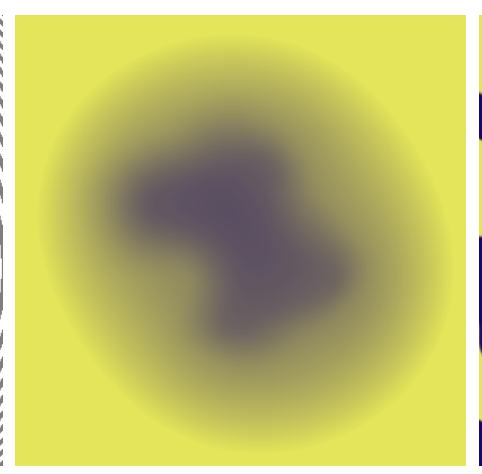

(e) Field Creation

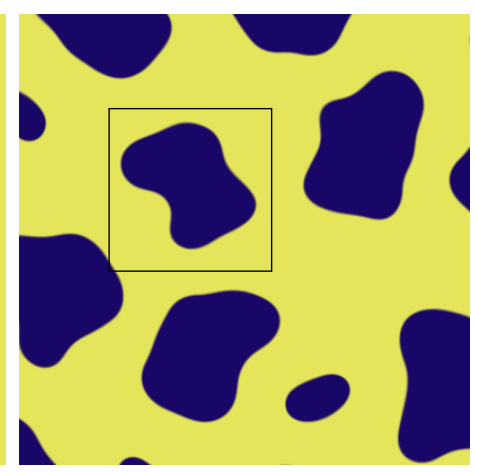

(f) Region Merging

Figure 4.1: From left to right, top to bottom, the six major steps of our algorithm: (a) mesh generation, (b) label assignment, (c) point grouping, (d) contour definition, (e) field creation, and (f) region merging. Each field created in step (e) is combined together to form the texture in step (f).

We start by constructing a mesh over the domain of our desired texture. We create the mesh by distributing a set of points on the plane, and applying a triangulation to the produced point set.

We next assign a label to each triangulated point of our mesh. A label is defined as a unique identifier with an associated color. The color of the labels are used to determine the colors of the texture. 
Once labels are assigned to the points of the mesh, we divide the points into different groups. Each group consists of points which all share the same label, and are all connected to each other in the mesh. An implicit region will be created for every group.

To define an implicit region, we derive two different sets of mesh edges from each group of points. The two sets of edges define the interior and exterior contour. The interior contour connects outer points of the group together, while the exterior contour connects points that are neighbours of the group. The implicit region is defined to be between the interior and exterior contour.

We then construct a smooth signed distance field for each contour. We define an implicit region by blending together the fields of both contours. By using a pair of distance fields to define a region, we are able to create an implicit field that is $C^{1}$ continuous.

The implicit regions of all groups are combined together to form a texture. A simple binary texture can be created by separating regions into either background or foreground. Pixels within any foreground region are assigned the same color, and all remaining pixels are assigned an alternate color.

Because each region is defined using an implicit field, we can also create more sophisticated textures by blending regions together, or by expanding or contracting the boundaries of different regions. Sections 4.1.1 to 4.1.6 give a detailed explanation of each step of our algorithm, and Sections 4.2 to 4.6 describe the effects of varying the different algorithm parameters.

\subsubsection{Mesh Generation}

The first step of our algorithm distributes a set of points with labels on the plane, and triangulates those points to form a mesh. The edges of the mesh will later be used to define region boundaries in the texture.

A simple method for creating a mesh is to distribute points in a Poisson disk distribution, and then to triangulate those points using a Delaunay triangulation. A Poisson disk distribution is defined as the limit of a uniform sampling process with a minimum-distance rejection criterion [36], and the Delaunay triangulation maximizes the minimum angle of the triangles in the mesh.

A mesh produced using a Poisson disk distribution and Delaunay triangulation will have all triangles be roughly the same size. Triangles with small angles will not be 
produced. Our method uses this type of mesh to generate many different unstructured patterns, such as the patterns found in certain toads and frogs. Alternative mesh construction methods may be more suitable depending on the type of texture we want to generate. Section 5.4 discusses how to create smooth patterns using more regular point distributions.

To simplify the discussion of our method and the various parameters, we assume that points are distributed in $[0,1]^{2}$ in $\mathbb{R}^{2}$ with the origin in the top-left corner. We will create a square texture by regularly sampling from within this unit square.

\subsubsection{Label Assignment}

After we have created our mesh, every point is assigned a label. A label is a unique identifier with an associated color. The final texture will be colored depending on the colors of the labels.

We can produce different textures by varying the labeling strategy. A simple labeling strategy is to randomly assign a point one of two different labels, which will give a simple organic pattern. We can produce different textures by changing how labels are assigned to points, and we discuss different labeling strategies in Section 4.4. More sophisticated labeling strategies traverse the edges of the mesh to produce various spot and stripe patterns.

\subsubsection{Point Grouping}

Once labels are assigned to the points on the mesh, we divide the points into different groups. Each group of points consists of points which all share the same label, and are all connected to each other in the mesh.

More formally, let $G$ be the graph of the original triangulation, and let $G^{\prime} \subseteq G$ be a subgraph obtained by deleting all edges whose endpoints are assigned different labels. The groups consist of the connected components in $G^{\prime}$.

Every point in a group shares the same label, and we define the label of a group as the label of its underlying points. Each group of points will be used to create a region in the texture.

An illustration of the first three steps of our algorithm is shown in Figure 4.2. The example mesh consists of eight points that have been triangulated using the Delaunay triangulation. The four points that lie on the boundary of the mesh are given the 


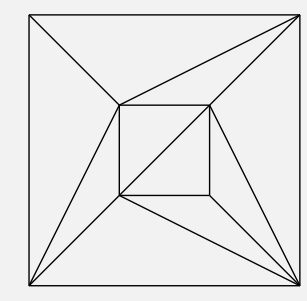

(a) Mesh Generation

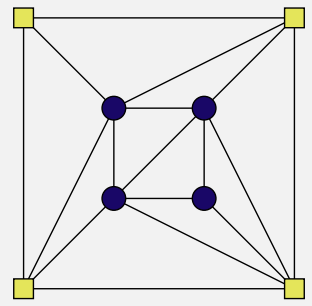

(b) Label Assignment

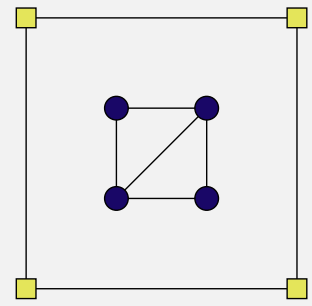

(c) Point Grouping

Figure 4.2: Initial steps of our method. (a) Mesh generation (b) Label Assignment (c) Point Grouping

same label, and the four points that lie on the interior of the mesh are given a different label. The labeled points are then grouped into two different connected components.

\subsubsection{Contour Definition}

For each group of points defined in the last step, we derive two subsets of edges from the mesh $G$. We refer to the two subsets of edges as the interior and the exterior contour.

The interior and exterior contour are used together to define an implicit region in the texture, with the contours defining an inner and outer boundary for that region. The interior contour connects points in a group together to form an inner boundary, while the exterior contour connects points adjacent to the group to form an outer boundary. Any point within the interior contour belongs to the region, and any point outside the exterior contour does not.

Let $B$ be the set of points belonging to a group, and let $C$ contain the neighbours of $B$ not belonging to $B$ itself. Assuming all groups consist of at least two points, the exterior contour is the set of all edges whose endpoints are in $C$.

Let $D$ be a set of points, such that each point is both in $B$ and adjacent to a point in $C$. The interior contour is the set of all edges whose endpoints are in $D$. The interior and exterior contours of a simple mesh are shown as a solid line in the middle and right images of Figure 4.4.

To extend the definition of the interior and exterior contour to include connected 
components of a single point, we make the following modifications. We include in the exterior contour every point whose neighbors are all in $B$, but do not belong to $B$ themselves. If the set $B$ consists of a single point, the interior contour is defined as that point. Figure 4.3 shows the interior and exterior contours for a set of points on a mesh (points are indicated as circles).

We next compute a smooth signed distance field for each interior and exterior contour. To compute the smooth signed distance field for a contour, we first compute the smooth unsigned distance field using the method of Biswas [11]. A detailed description of this procedure is found in Section 3.6.

We obtain the sign of the distance field by using the faces of $G$. Let $\Omega_{E}$ be the polygonal region defined as the union of all faces in $G$ incident to any point in $C$. Let $\Omega_{I}$ be the polygonal region defined as the union of all faces in $G$ that have every point in the face belonging to $C$. The boundaries of $\Omega_{I}$ and $\Omega_{E}$ will lie on the interior and exterior contours respectively for a group of points.

For the exterior contour, the field value at a location $p$ is positive if $p \in \Omega_{E}$ and negative otherwise. For the interior contour, the field value at a location $p$ is negative if $p \in \Omega_{I}$ and positive otherwise. We denote $d_{E}(p)$ and $d_{I}(p)$ as the smooth signed distance functions for the exterior and interior contours of $C$.

Figure 4.6 shows a graphical representation of $d_{I}(p)$ for the interior points of a simple mesh. Figure 4.6(a) maps values of $d_{I}(p)$ onto colors. Positive values of $d_{I}(p)$ are indicated in light yellow, and negative values are indicated in dark blue. The isocontours of $d_{I}(p)$ are shown in Figure 4.6(b). A corresponding visualization of $d_{E}(p)$ for the same set of points is shown in Figure 4.7.

Figure 4.5 shows a specific cross-section of the visualized field. The cross-section area is shown as a dashed line on the mesh image. The $x$-coordinate of the texture is shown on the horizontal axis of the graph, and the distance value is shown on the vertical axis. The distance field of the interior contour is shown in black, and the distance field of the exterior contour is shown in gray. The corresponding exact distance fields are shown as a dashed line. In the next step, we use the distance fields of both contours to define a single smooth signed distance field for an implicit region in the texture. 


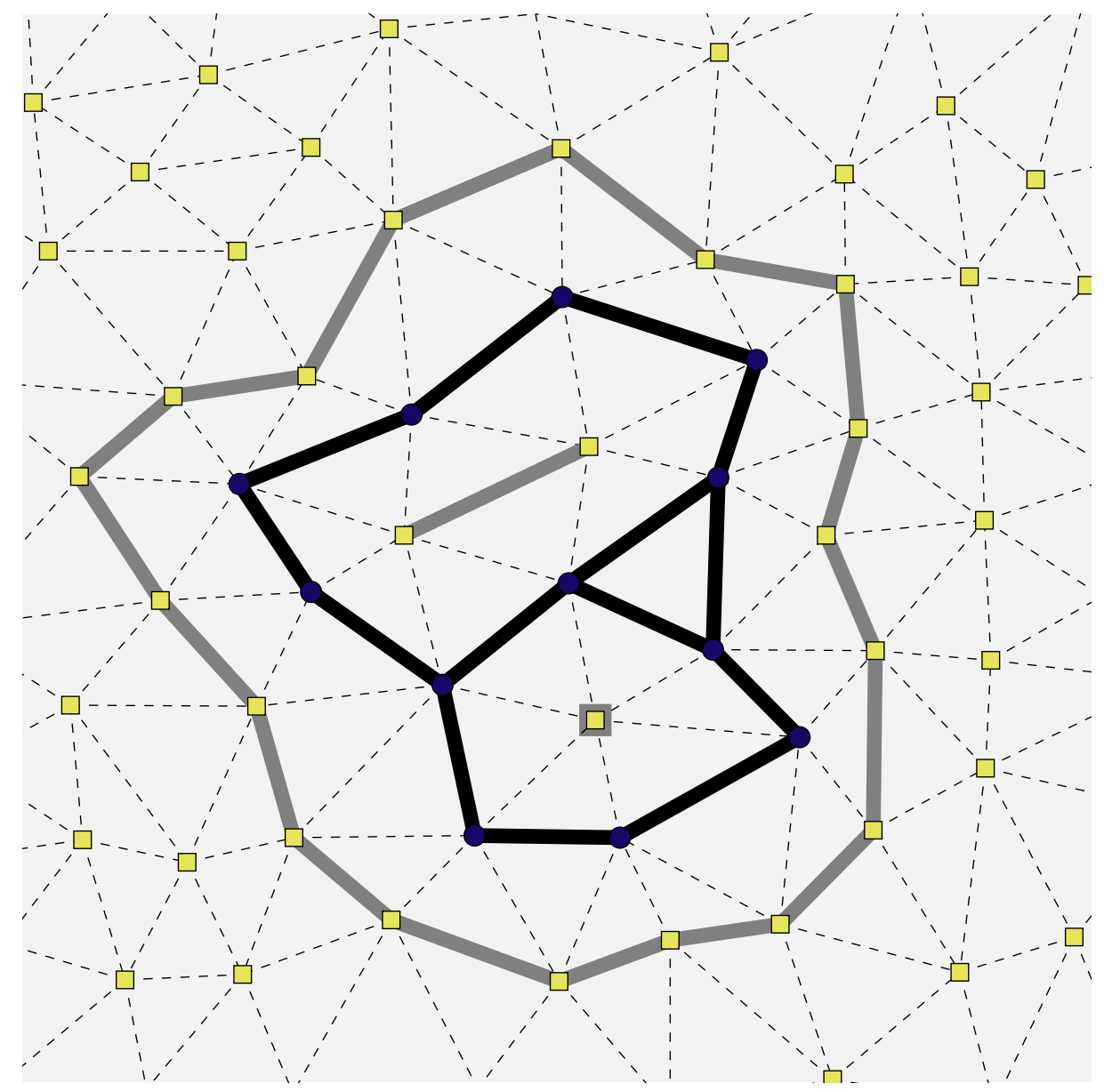

Figure 4.3: Interior and exterior contours for a set of points. The interior contour is shown in black, and the exterior contour is shown in gray. The exterior contour consists of two sets of connected line segments, and a single point.

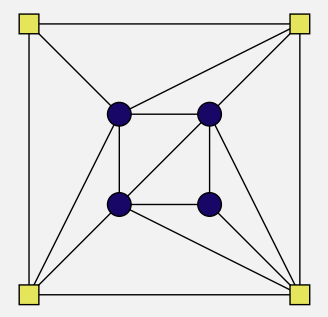

(a) Original Mesh

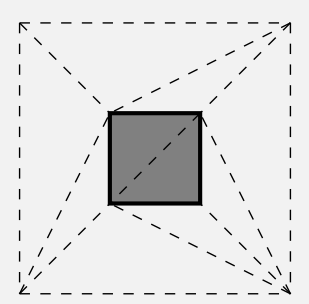

(b) Interior Region $\Omega_{I}$

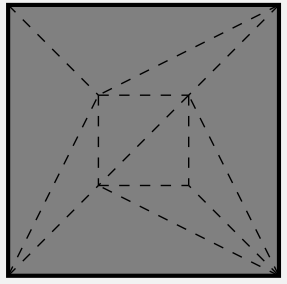

(c) Exterior Region $\Omega_{E}$

Figure 4.4: Polygonal regions of the interior points of a mesh, with regions highlighted in gray. (a) Labeled Mesh (b) Region $\Omega_{I}$ (c) Region $\Omega_{E}$ 

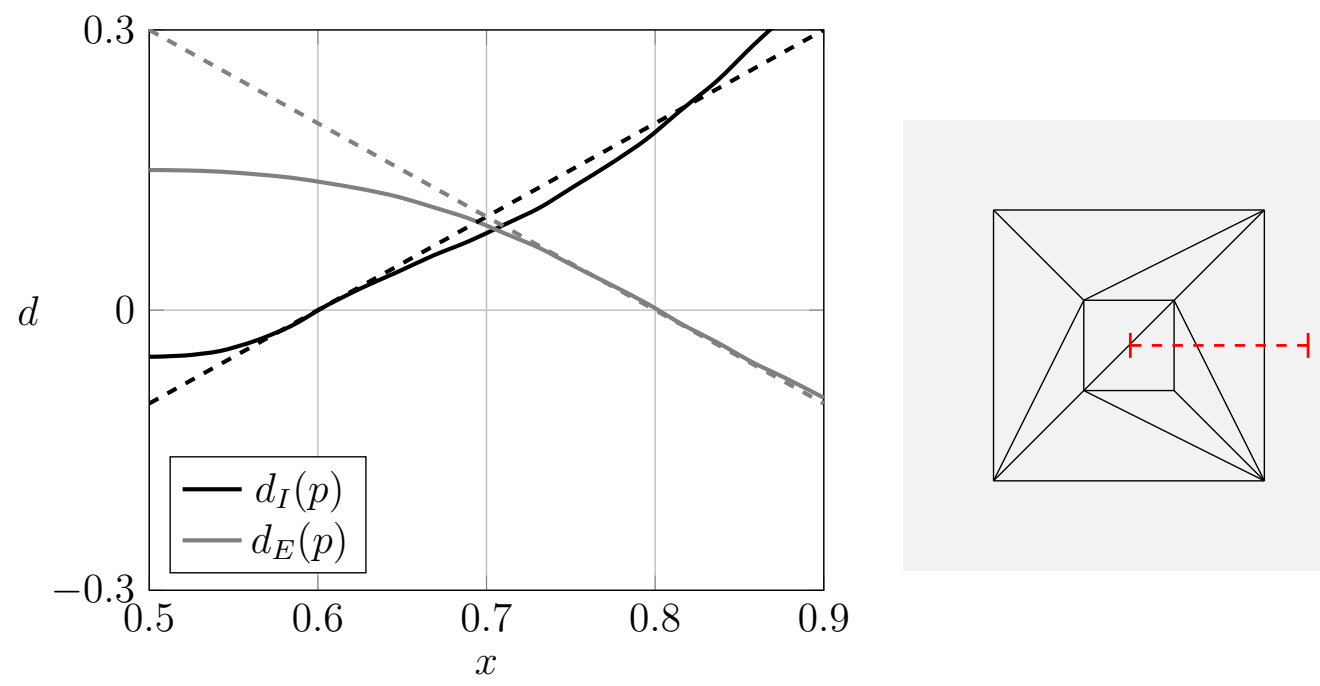

Figure 4.5: Graphical representation of $d_{I}(p)$ and $d_{E}(p)$ for the interior points of a simple mesh, with the corresponding exact distance fields shown as dashed lines. The cross-section area is shown as a dashed line on the mesh image.

\subsubsection{Field Creation}

There are several different methods for defining an implicit region. Skeletal implicit surfaces define an implicit region by specifying a particular offset distance from a set of modeling primitives. The set of primitives is known as the skeleton, and the skeleton lies within the defined region. An example of skeleton-based modeling was given in Section 2.7, which described using implicit point primitives to form a texture.

Another approach for defining an implicit region is to specify the boundaries of a region with an arbitrary polygon. The specified polygon is referred to as the cage of the implicit surface. Section 3.4 described using double-layered potentials to define the cage of an implicit surface. Points on the interior of the cage would have a negative charge, and points on the exterior would have a positive charge. The implicit surface is defined as some offset from the zero set of the produced distance field.

Unfortunately, neither skeletal implicit surfaces nor double-layered potentials produce a field that is smooth everywhere. Skeletal implicit surfaces have gradient discontinuities occurring along the medial axis of the skeleton. As described in Section 3.1, the gradient discontinuities are a consequence of using exact distance fields to define the surface. For surfaces created using double-layered potentials, the gradient field is discontinuous at the zero set where two line segments meet. 


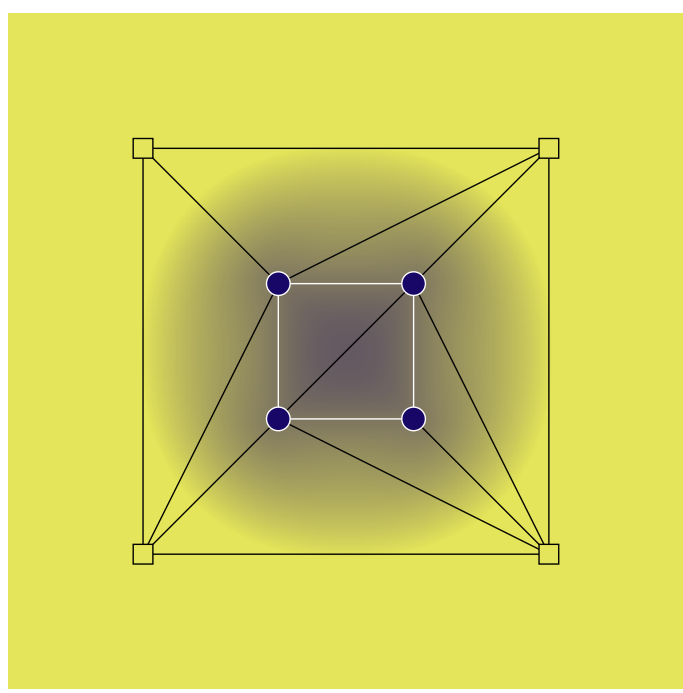

(a)

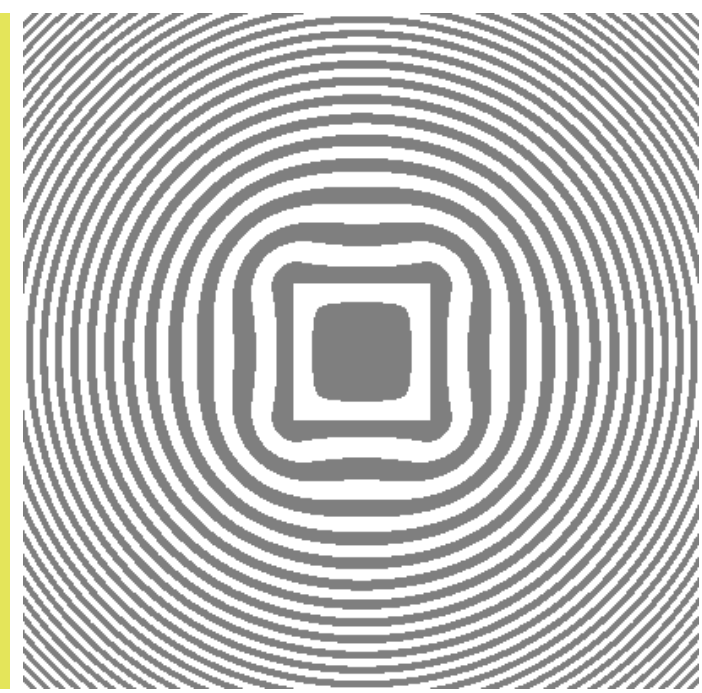

(b)

Figure 4.6: Smooth signed distance field of the interior contour (highlighted in white) for the interior points of a mesh with (a) color representation of distance, and (b) isocontours of the produced field.

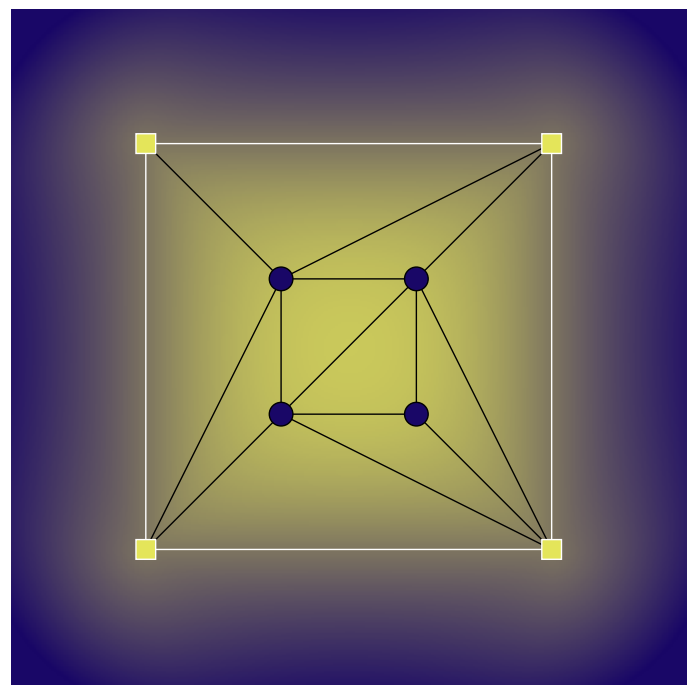

(a)

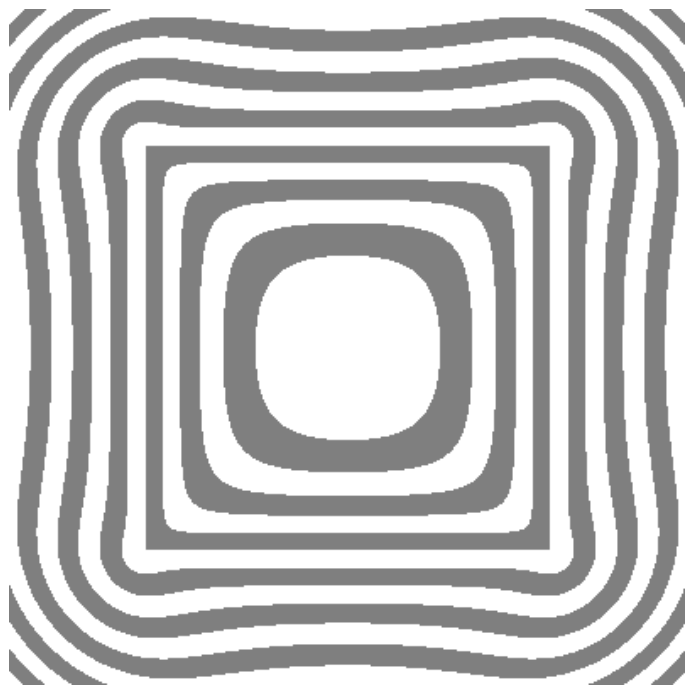

(b)

Figure 4.7: Smooth signed distance field of the exterior contour (highlighted in white) for the set of interior points of a mesh with (a) color representation of distance, and (b) isocontours of the produced field. 
Our approach for defining an implicit region is a combination of both skeletonbased and cage-based approaches. We interpret the interior and exterior contours as defining the inner and outer boundaries of a defined region. We blend the distance fields of each contour together to produce a distance field for the region.

Both interior and exterior contours are used because we desire a combined distance field that is smooth everywhere. A single contour is insufficient because the gradient field of a single contour is discontinuous at the zero set where two line segments meet. For example, Figure 4.6 has gradient discontinuities occurring at each interior point of the mesh.

The combined distance field of a region is based on computing the signed distance away from a curve approximately midway between the interior and exterior contour. We define this midway curve as:

$$
\partial \Omega=\left\{p \in \mathbb{R}^{2} \mid d_{I}(p)-d_{E}(p)=0\right\} .
$$

A possible signed distance function that estimates the distance from the midway curve is given by

$$
d_{M}(p)=\frac{d_{I}(p)-d_{E}(p)}{2} .
$$

This distance function is positive near the exterior contour, and becomes negative near the interior contour. The zero set of this distance function also matches that of the midway curve. Unfortunately, the combined distance field $d_{M}(p)$ is not smooth everywhere, and discontinuities exist wherever the interior or exterior contours are discontinuous. Specifically, $d_{M}(p)$ is discontinuous at the intersection point of any two edges of a contour.

We next describe an alternative distance function to produce an everywhere smooth field, also based on estimating the distance from the midway curve. To avoid gradient discontinuities, we do not use the distance field of a contour near its zero set. Instead, we use the distance field of the opposing contour to estimate the distance from the midway curve.

At the exterior contour, we approximate the distance to the midway curve as $d_{I}(p) / 2$. At the interior contour, we approximate the distance to the midway curve as $-d_{E}(p) / 2$. We use a smoothstep function $s(x)$ to blend between approximations. 

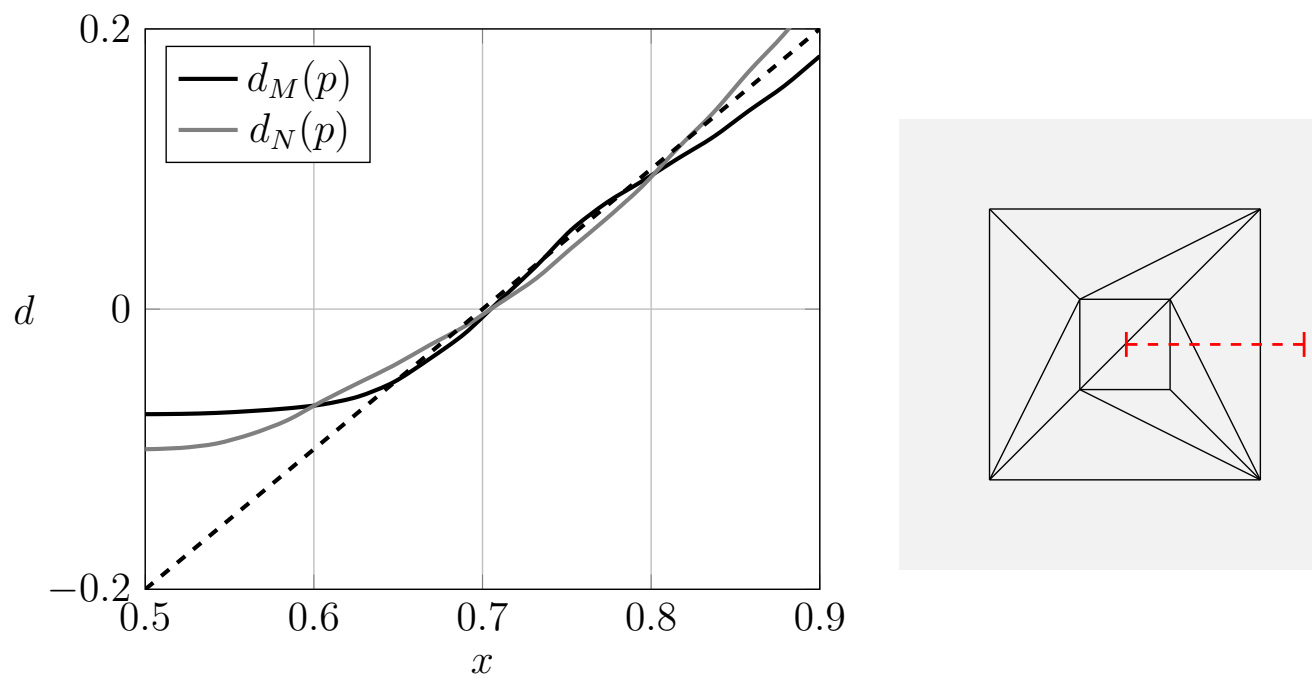

Figure 4.8: Cross-section of $d_{M}(p)$ and $d_{N}(p)$ for the interior points of a simple mesh, with the exact distance field midway between contours shown as a dashed line. The cross-section area is shown as a dashed line on the mesh image.

The combined distance function is defined as

$$
d_{N}(p)=w_{E} \frac{-d_{E}(p)}{2}+w_{I} \frac{d_{I}(p)}{2}
$$

where

$$
\begin{aligned}
& w_{E}=s\left(1-d_{I} /\left(d_{I}+d_{E}\right)\right) \\
& w_{I}=s\left(d_{I} /\left(d_{I}+d_{E}\right)\right) .
\end{aligned}
$$

The smoothstep function we use is a fifth order polynomial originally proposed by Perlin [64], given by

$$
s(x)=\left\{\begin{array}{ll}
6 x^{5}-15 x^{4}+10 x^{3} & 0<x<1 \\
0 & x \leq 0 \\
1 & x \geq 1
\end{array} .\right.
$$

This smoothstep function gradually transitions between unity and zero, and has zero first and second derivatives at both ends of the transition.

The function $d_{N}(p)$ has the same zero set as $d_{M}(p)$, and also behaves as a smooth 
signed distance field for the midway curve. We show a comparison between the two methods we have presented in Figure 4.9 and Figure 4.10 for the interior points of a simple mesh. Notice that there are gradient discontinuities present in $d_{M}(p)$ at each vertex in the mesh, but that these discontinuities are not present in $d_{N}(p)$.

Figure 4.8 shows a specific cross-section of the visualized field. The $x$-coordinate of the texture is shown on the horizontal axis of the graph, and the distance value is shown on the vertical axis. The function $d_{M}(p)$ is shown as a solid gray line, and $d_{N}(p)$ is shown as a solid black line. The exact distance field for the interior contour is shown as a dashed line on the graph.

\subsubsection{Region Merging}

After we have created a smooth distance field for every group of points, we combine the distance fields together to create a texture. Different textures can be created depending on how regions are combined. As an example, we now describe a simple method of merging regions together to produce a binary texture.

We assume that each point in the original graph is assigned either a foreground label or a background label. Let $\left\{F_{0}, F_{1} \ldots F_{n}\right\}$ be the set of connected components which are all assigned a foreground label.

For every $F_{i}$ with combined distance functions $d_{N}(p)$, we create a foreground region $R_{i}$. This region is defined as the union of all locations $p$ such that $d_{N}(p)<0$, and represents the interior of the midway curve. Every point within a foreground region is assigned the foreground color, and all other points are assigned the background color.

Let $c_{f}$ be the color associated with the foreground label, and let $c_{b}$ be the color associated with the background label. The color of the output texture at some location $p$ is given by

$$
f(p)= \begin{cases}c_{f} & \exists i: p \in R_{i} \\ c_{b} & \text { otherwise }\end{cases}
$$

Examples of textures produced by this method are shown in Figure 4.11, with the texture for our example mesh shown in Figure 4.11(a). The texture in Figure 4.11(b) was created by distributing points in a Poisson disk distribution, and randomly assigning a point to have either a foreground or background label. 


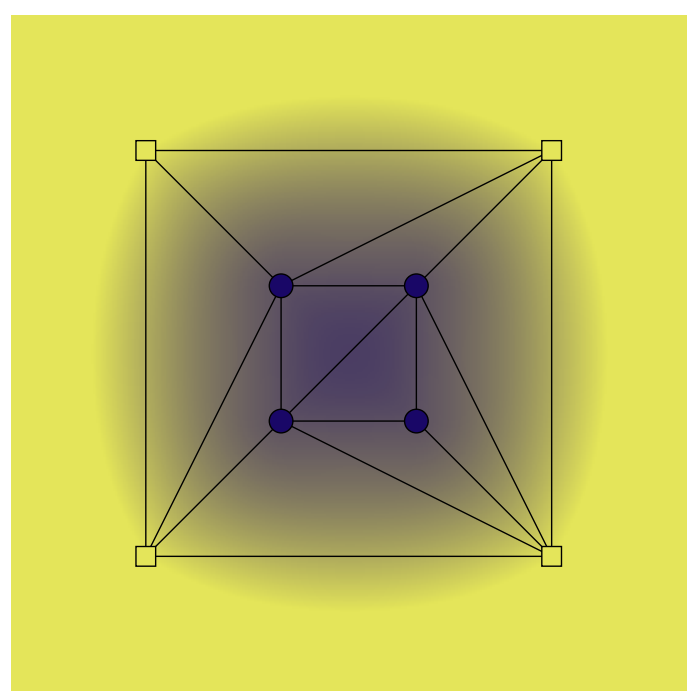

(a)

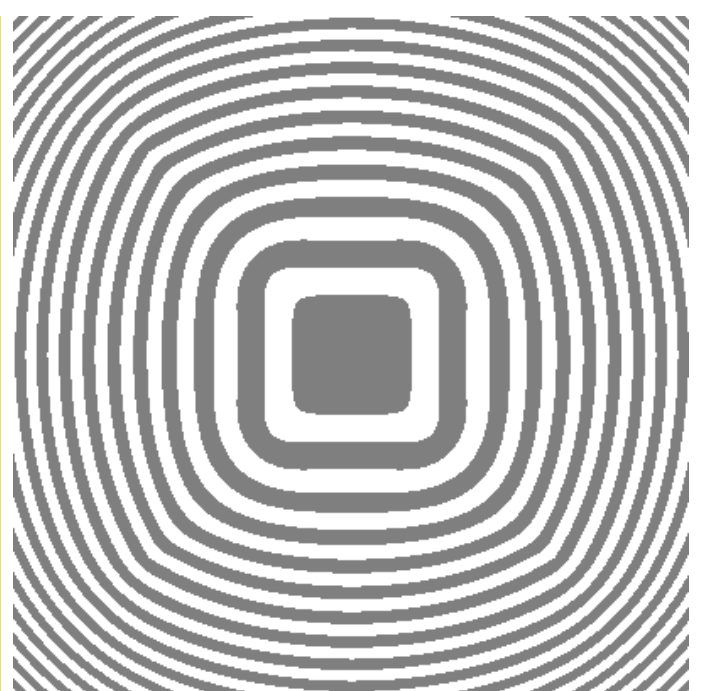

(b)

Figure 4.9: Distance field $d_{M}(p)$ with gradient discontinuities. (a) color representation of distance, and (b) isocontours of the produced field.

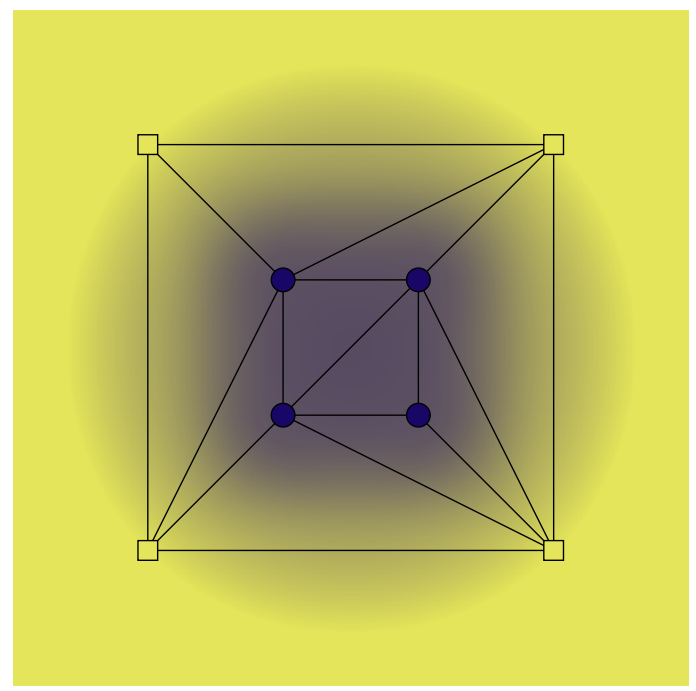

(a)

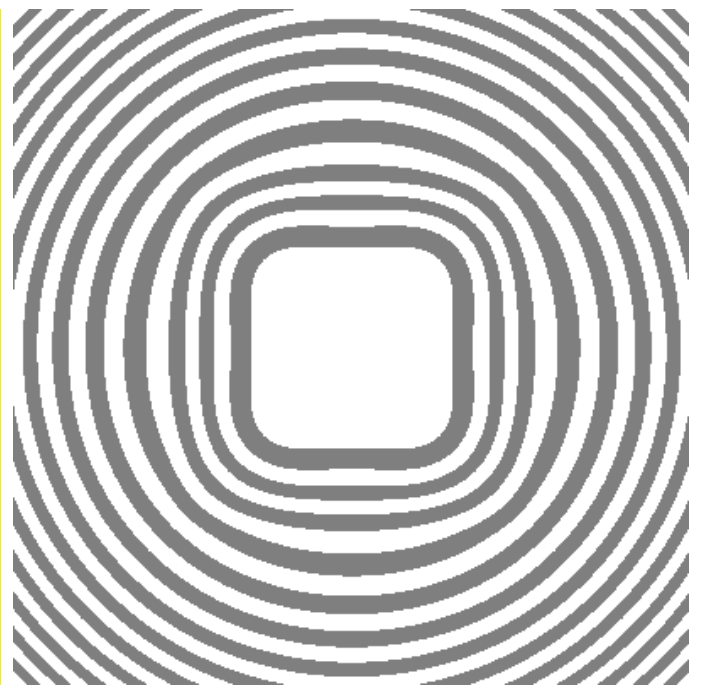

(b)

Figure 4.10: Distance field $d_{N}(p)$ that is $C^{1}$ continuous. (a) color representation of distance, and (b) isocontours of the produced field. 


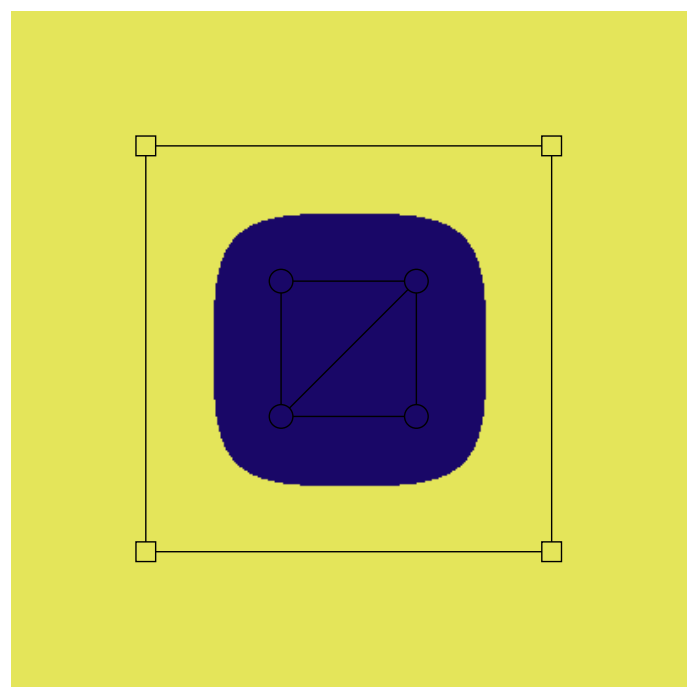

(a)

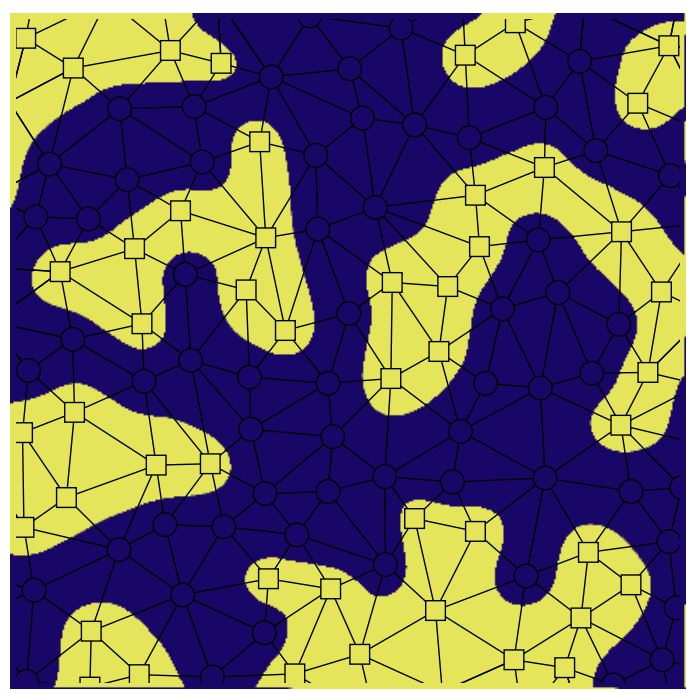

(b)

Figure 4.11: Merging implicit regions to form a textures. (a) simple texture, and (b) random binary texture.

We can use the distance fields of each region to create more elaborate effects. In the next section, we show how to blend regions together by deriving a weight function from each distance field.

\subsection{Blending}

To produce a smooth transition between regions in our texture, we use the distance function of the region to define a weight function. The weight functions of all regions are blended together to produce the texture.

Let $C=\left\{C_{0}, C_{1} \ldots C_{n}\right\}$ be the set of connected components in our mesh. For each connected component $C_{i}$ with associated color $a_{i}$, we define an associated weight function $w_{i}(p): \mathbb{R}^{2} \rightarrow \mathbb{R}$. The color of the blended output texture at some location $p$ is given by

$$
f(p)=\frac{\sum_{i=1}^{n} w_{i}(p) a_{i}}{\sum_{i=1}^{n} w_{i}(p)}
$$

Our texture function $f(p)$ then becomes a weighted average of color values from each connected component. Other possibilities of combining the different weight functions can be considered, such as selecting the color with the largest total weight.

To define our weight function $w_{i}(p)$, we use the distance function $d_{N}(x)$ of the 
connected component $C_{i}$. We introduce a parameter $r$ representing a blending radius away from the midway curve $d_{N}(p)=0$. The fraction $d_{N}(p) / r$ is exactly -1 if $p$ is $r$ away from the midway curve in the direction of the interior contour, and +1 away in the direction of the exterior contour. We next scale this fraction to the range $[0,1]$ to give the function:

$$
h_{N}(p)=\frac{d_{N}(p) / r+1}{2} .
$$

The weight function $w_{N}(p)$ is defined as the composition of $h_{N}(p)$ with a smoothstep function $s(x)$, and is given by

$$
w_{N}(p)=s\left(h_{N}(p)\right),
$$

where the definition of $s(x)$ is given in Section 4.6. The smoothstep function is used to smoothly transition between the weights of the different connected components.

Figure 4.12 shows a set of textures where each texture has a different specified blending radius. Figure 4.13 shows a cross-section of the different blended textures in Figure 4.12. The set of textures with a blending radius $r=0,0.05$, and 0.10 are shown as a solid, dashed, and dotted line.

\subsection{Offset Distance}

We can use the distance function $d_{N}(p)$ to expand or contract an implicitly defined region. For a connected component $C$ with distance function $d_{N}(p)$, we define an implicit region $R$ as

$$
R=\left\{p \mid d_{N}(p)<K\right\} .
$$

The variable $K$ can be interpreted as an offset distance, with the region being defined as a shifted offset from the midway contour. Inflation of the region $R$ occurs if $K$ is positive, and deflation occurs if $K$ is negative.

The behaviour of changing the offset distance can be described based on the interior and exterior contours of a region. When a region is expanded, the boundary more closely follows the distance field of the interior contour. When a region is contracted, the boundary more closely follows the distance field of the exterior contour.

In Figure 4.14, we show a set of implicit regions created with different offset distances for the interior points of a simple mesh. A cross-section of the distance field 


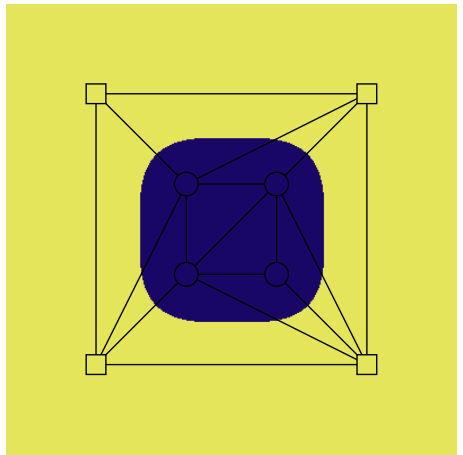

(a) $r=0$

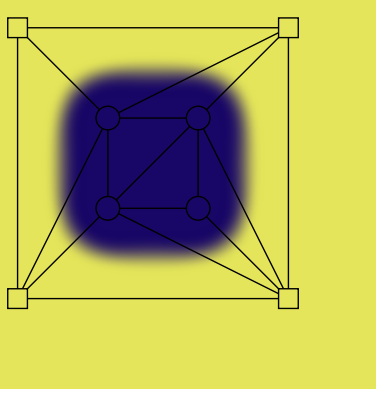

(b) $r=0.05$

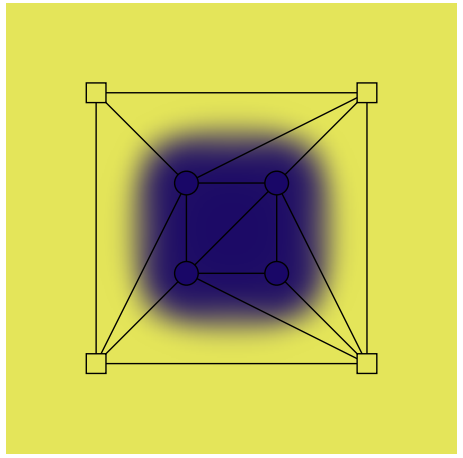

(c) $r=0.10$

Figure 4.12: Blending of implicit regions with different blending radius $r$.
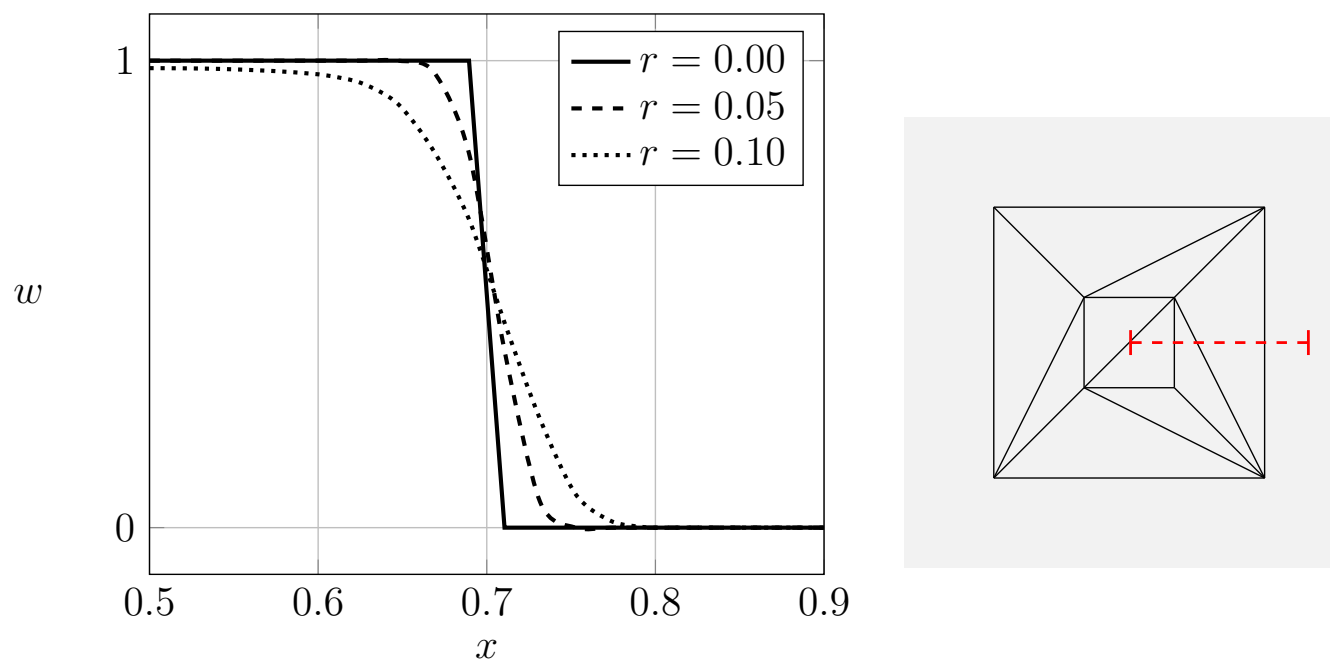

Figure 4.13: Cross-section of $w_{i}(p)$ with different blending radii. The cross-section area is shown as a dashed line on the mesh image. 
$d_{N}(p)$ of the mesh is shown in Figure 4.15, with different offset distances highlighted as a dashed line. The boundary of the implicit region is where the distance field intersects the offset distance, and is shown as a solid red line.

\subsection{Labeling}

\subsubsection{Stripes and Spots}

This section discusses different methods for assigning labels to points in the graph. We have shown that a random binary labeling of points will produce an appealing organic image, but more sophisticated patterns can be generated by taking into account the underlying graph. Caron and Mould [18] considered different methods of reasoning over a graph in their work on partition of unity textures, and these methods can be directly applied to our algorithm. We reproduce their method of using a depth-first and breadth-first traversal of the graph to create stripe and spot patterns.

We generate stripe patterns as follows. We select a random point $p$ in the graph and a fixed number $k$ representing the length of the stripe. We mark $p$ with a foreground label, and add its unlabeled neighbors to the front of an exploration list. To grow the stripe, we iteratively repeat the previous step by removing a point from the front of the exploration list, marking it as the foreground label, and adding unlabeled neighbors to the front of the exploration list. The stripe stops growing when it is of size $k$, or when the exploration list is empty. Once the stripe ceases growing, we mark all remaining points in the exploration list with the background label. We repeat this process until all points are labeled.

We generate spot patterns by modifying how stripe patterns are generated. Specifically, when neighbors are added to the exploration list, we add to the back rather than the front. The labeling procedure has the effect of forming clusters resembling spot patterns. Figure 4.16 shows a stripe and spot pattern created using our algorithm.

\subsubsection{Graph Traversal}

We briefly mention other graph-based labeling strategies. Round-robin clustering [18] was previously explored to generate organic but irregular structures. In this method, a set of center points sequentially take turns applying a label to a point. Expansion 


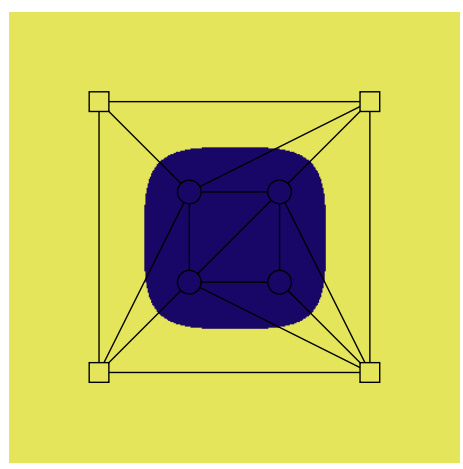

(a) $K=0$

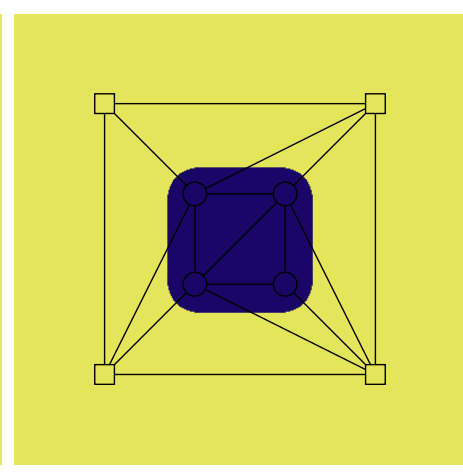

(b) $K=-0.05$

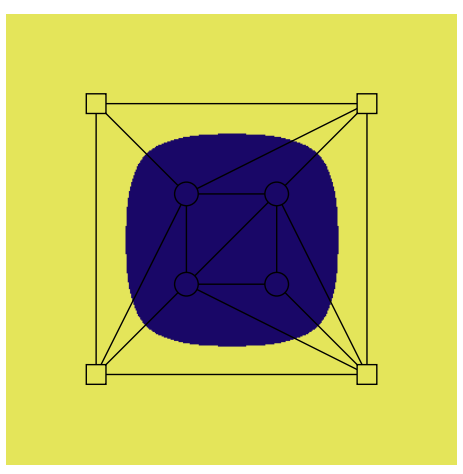

(c) $K=0.05$

Figure 4.14: Different offset distances $K$ to expand or contract a region.
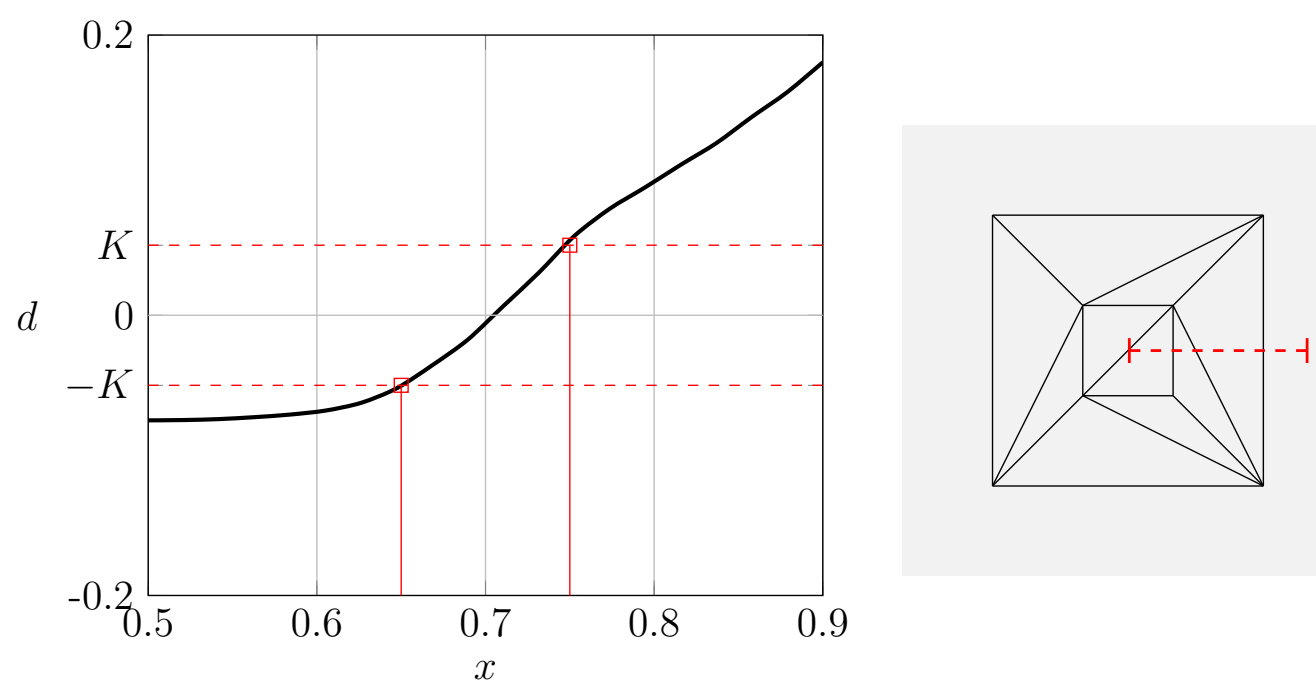

Figure 4.15: Cross-section of $d_{N}(p)$ showing different offset distances for a simple mesh. The cross-section area is shown as a dashed line on the mesh image. 
stops when there are no neighboring points to claim. The result is a connected but non-Voronoi like growth pattern.

Branching structures can be created by labeling paths between points using the same label. In this method, a set of initial points are selected, and an approximate minimum spanning tree is constructed using the method of Mehlhorn [54]. Every point belonging to the spanning tree is labeled with a foreground label, and all other points are assigned the background label. Figure 4.17 shows an example texture created by connecting a set of foreground points to form an approximate minimum spanning tree. We expect other graph labeling methods to produce their own interesting organic phenomena.

\subsection{Point Distribution}

The textures we created in the previous sections used the Poisson disk distribution. In a Poisson disk distribution, points are tightly packed, but are at least some specified distance apart. This distribution is convenient because it provides a relatively uniform distance to blend between regions, as well as a uniform level of detail in the texture, while still being able to generate irregular patterns without lattice artifacts.

Roughly speaking, the density of points in the texture controls the level of detail. Changing the point density of our algorithm can be done by varying the minimum distance parameter $d$ of the Poisson disk distribution. In Figure 4.18, we generate a set of random binary patterns with different minimum point densities $d$. Varying the point density of our algorithm has the effect of scaling the texture, with a smaller Poisson disk spacing creating a more detailed texture.

We can use other point distributions, and our method will still produce smooth boundary curves. A texture generated with a purely random uniform point distribution is shown on the left in Figure 4.19. While there are sharper changes in curvature, the boundary curves of the texture remain continuous

To produce varying levels of detail in a texture, we vary the feature point distribution across the domain. We use a density map, which is an intensity image specifying point density, to vary the detail arbitrarily. We take the density map and apply an adaptive sampling algorithm [60] to generate the point locations. An example of varying the point density is shown on the right image of Figure 4.19. Using a density map allows us to easily generate non-stationary patterns, such as having a denser 


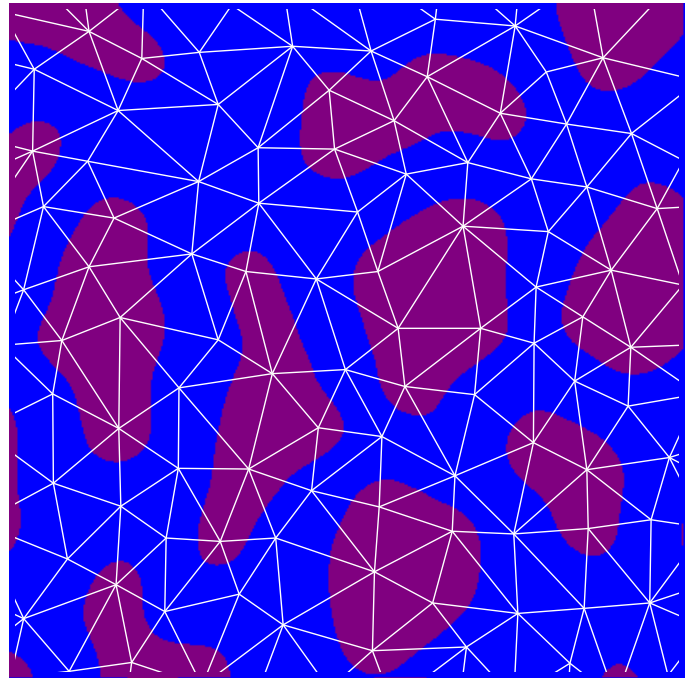

(a)

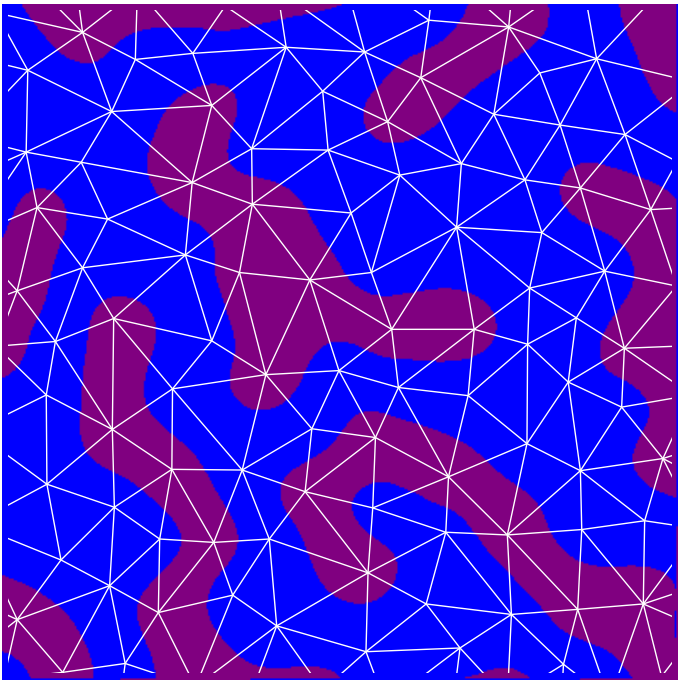

(b)

Figure 4.16: Stripe and spot patterns created with a graph-based traversal. (a) spot patterns, and (b) stripe patterns.

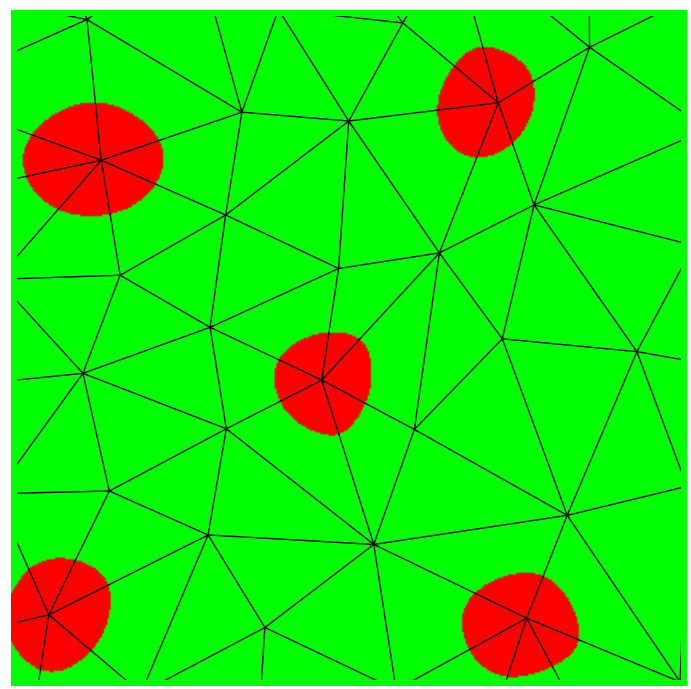

(a)

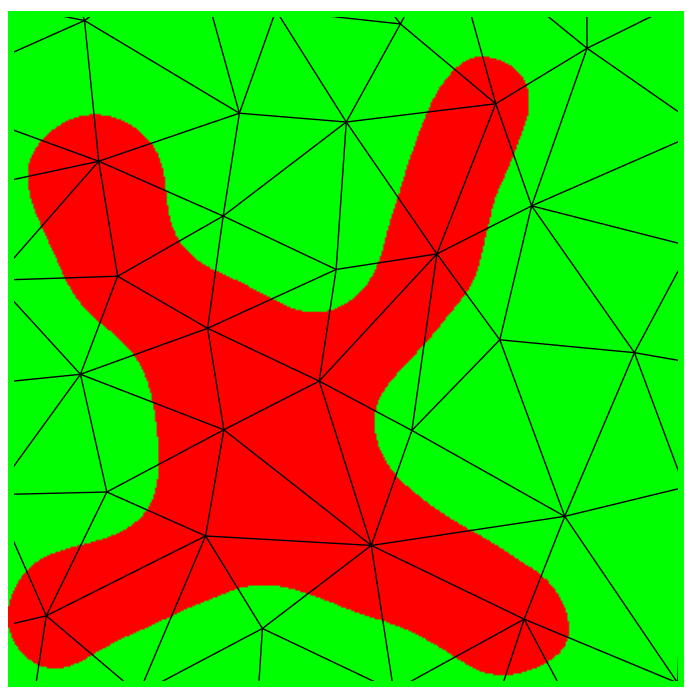

(b)

Figure 4.17: Branching structures created using a graph-based traversal. (a) texture with initial set of labeled points, and (b) texture with labeled points connected to form a minimum spanning tree. 
spot pattern along edge boundaries.

\subsection{Color Values}

We previously assumed labels were associated with color values, and an entire region was composed of a single color. More interesting patterns can be generated if the color varies across a region. We define $a_{i}(p)$ to be color of a region at location $p$ in the texture.

There are several different possibilities for how to vary the color within a region. One approach is to supply an image $I(p)$ to the texture function, and have the color of the region be the color of the image at each location such that $a_{i}(p)=I(p)$.

Another approach is to have the color depend on the distance function $d_{N}(p)$ or the weight function $w_{i}(p)$ of the region. We can use a color spline to map field values to the corresponding colors. A color spline is defined as an arbitrary mapping of an input value to an output color [25], and often uses continuous curves to provide a smooth transition between colors. Using a color spline with a distance functions allows us to create more sophisticated textures.

Figure 4.20 shows examples of using a color spline to add an outline to a spot texture. The color of the spot is the outline color if $d_{N}(p)<K$, and is the interior spot color otherwise. Varying the constant $K$ produces outlines of different thickness. A discontinuous color transition is shown in Figure 4.20(b), and a smooth color transition is shown in Figure 4.20(c).

\subsection{Summary}

In this chapter we described a method for constructing organic textures using smooth signed distance fields. In our method, a collection of points are grouped based on their labels and connectivity within a mesh. For each group of points, two smooth distance fields were used to define an implicit region. Implicit regions were then combined together to produce the output texture.

We showed how blending between regions can be controlled by modification of defined implicit fields, and how we could expand or contract an implicitly defined region. We also demonstrated how different textures can be generated by changing 


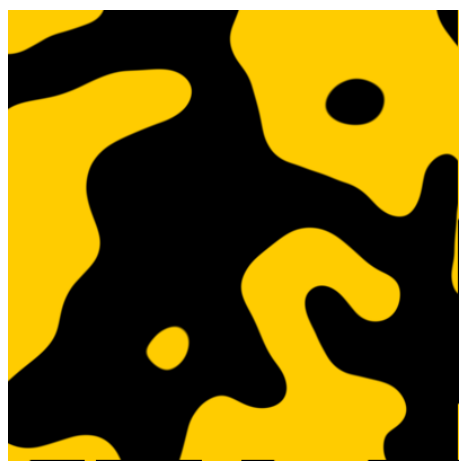

(a) $d=0.10$

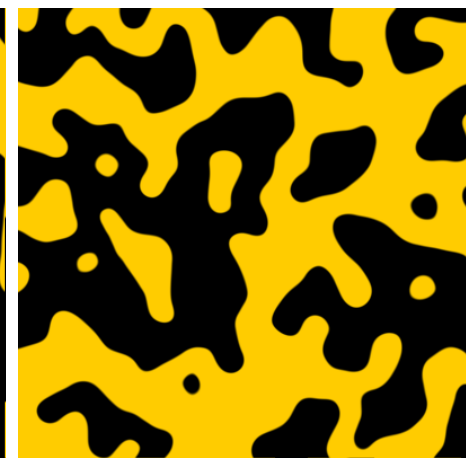

(b) $d=0.05$

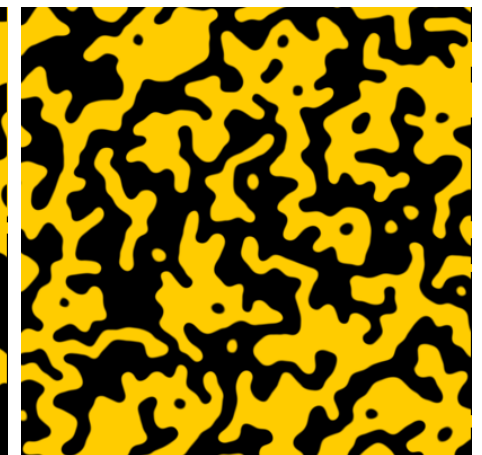

(c) $d=0.025$

Figure 4.18: Random binary textures with varying point densities $d$.

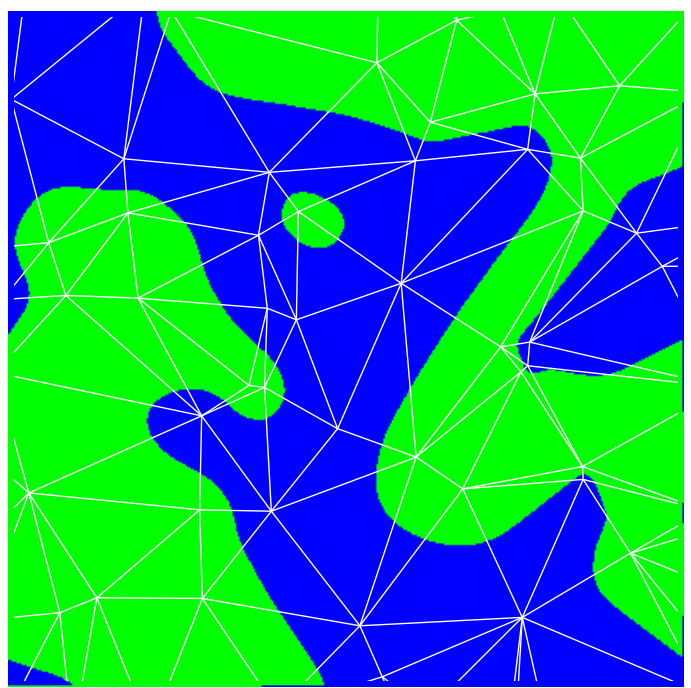

(a)

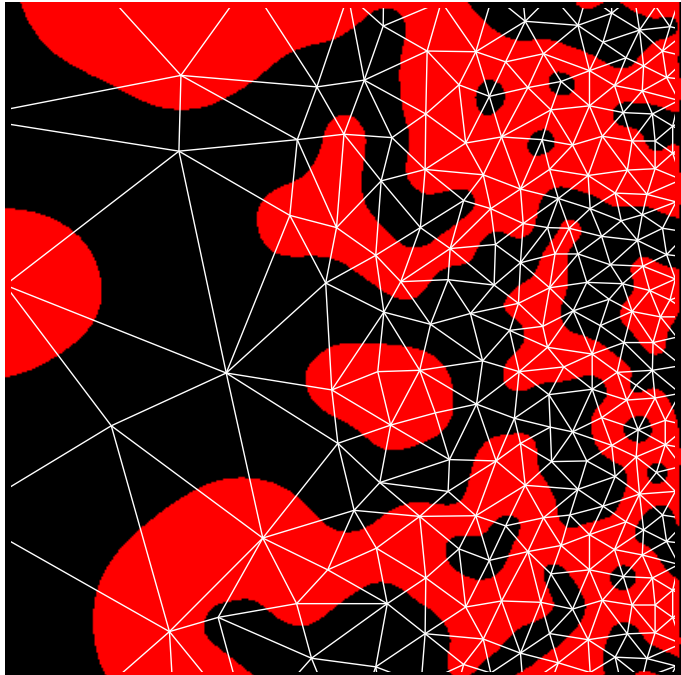

(b)

Figure 4.19: Different textures generated by varying the point distribution. (a) random uniform point distribution, and (b) varying density point distribution. 


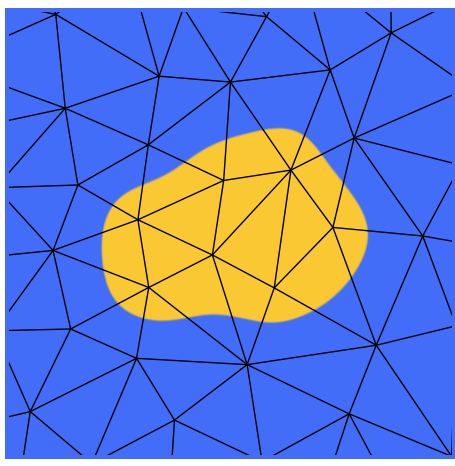

(a)

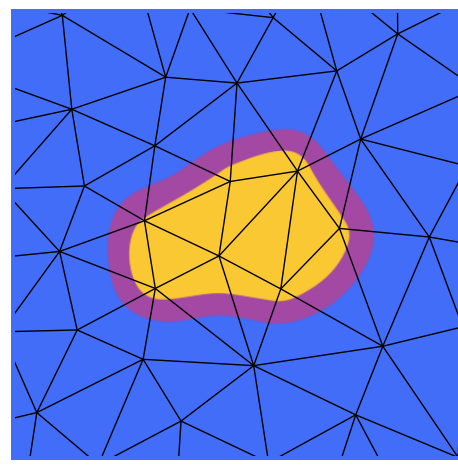

(b)

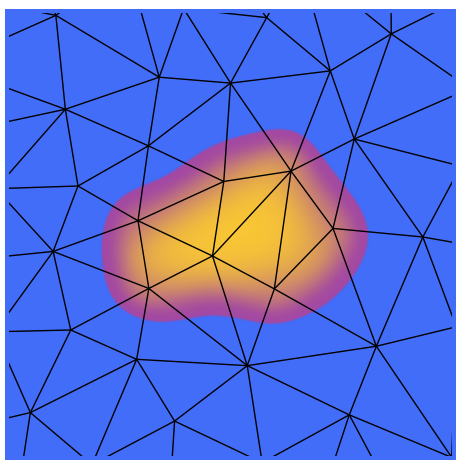

(c)

Figure 4.20: Textures produced with a color spline to produce an outline. (a) initial texture image, (b) discontinuous color transition, and (c) smooth color transition

how points are labeled and distributed across the domain. In the next section, we apply these different techniques to produce the organic patterns we desire. 


\section{Chapter 5}

\section{Results}

\subsection{Introduction}

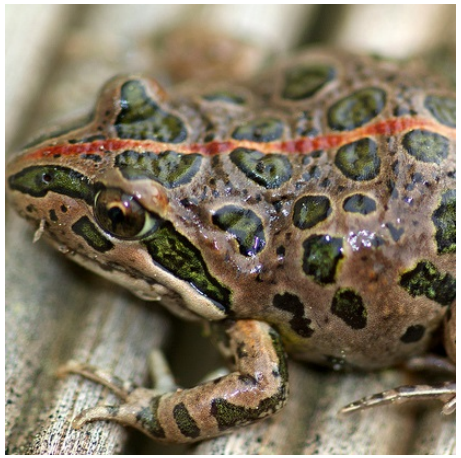

(a)

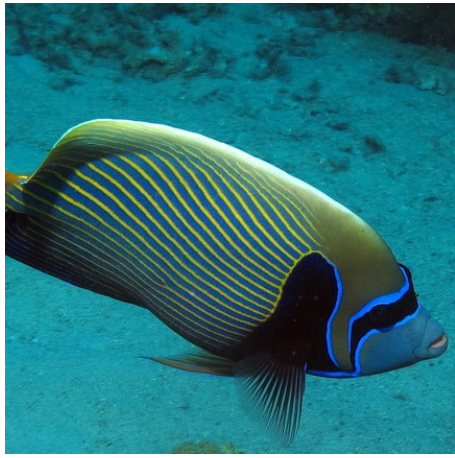

(d)

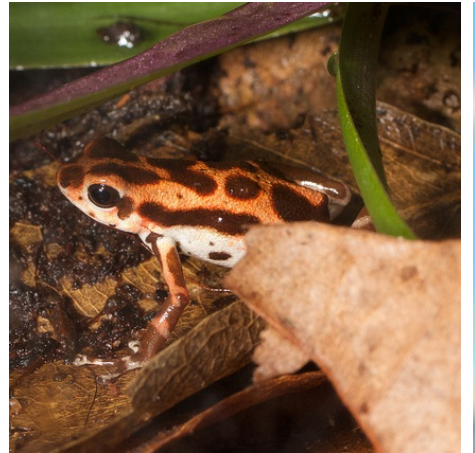

(b)

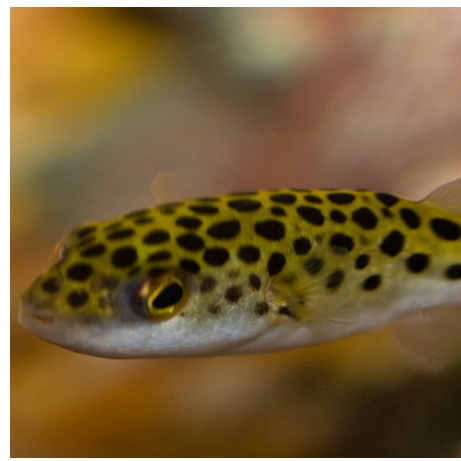

(e)

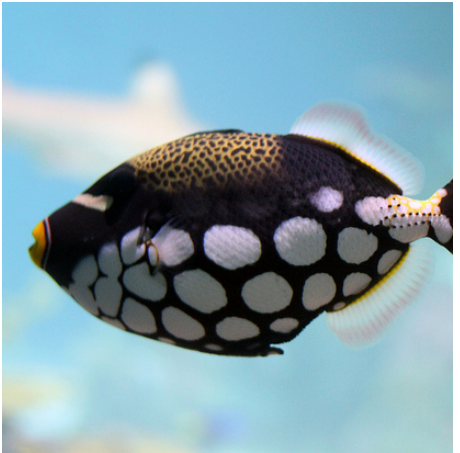

(c)

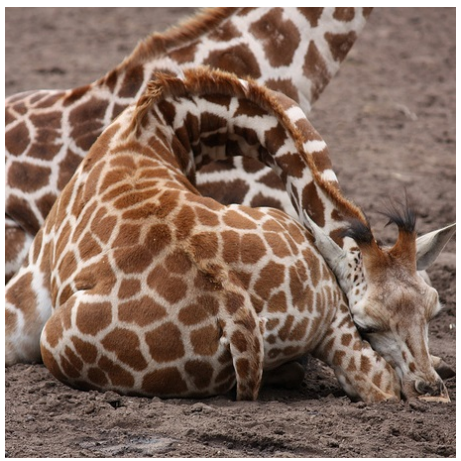

(f)

Figure 5.1: From left to right, top to bottom, examples of patterns found in the natural world: (a) spotted marsh frog [80], (b) strawberry poison-dart frog [34], (c) clown triggerfish [28], (d) emperor angelfish [39], (e) pufferfish [19], and (f) common giraffe [53]. 
In the previous chapter, we presented our method for generating organic textures using smooth signed distance fields. We showed how to produce a simple binary texture by distributing points in a Poisson disk distribution, randomly assigning a point one of two different labels, and having the texture depend on whether or not a pixel was in a foreground region.

This chapter will show how other kinds of textures can be created. Specifically, we will show how to produce different irregular patterns, smooth patterns, Voronoi patterns, and complex patterns. In the next section, we give a summary of the different parameters of our algorithm. The remainder of this chapter describes how to vary these parameters to produce specific kinds of textures. The patterns we generate are motivated by examples found in the natural world, as shown in Figure 5.1.

\subsection{Method Inputs}

The different inputs to our algorithm are listed below.

$G(V, E) \quad$ Mesh of the texture. The mesh consists of a set of points $V=$ $\left\{p_{0}, p_{1} \ldots p_{n}\right\}$ and a set of edges $E=\left\{e_{0}, e_{1} \ldots e_{n}\right\}$.

A Set of labels $A=\left\{a_{0}, a_{1} \ldots a_{n}\right\}$ in the texture.

$L \quad$ A labeling function $L: V \rightarrow A$. Each point $p_{i}$ in the mesh is associated with a label $a_{i}$.

d Minimum distance between any two points in the mesh, when points of the mesh have been distributed in a Poisson Disk distribution.

Blending radius of the texture.

$K \quad$ Offset distance of a region in the texture. Inflation of a region occurs if $K>0$, and deflation occurs if $K<0$.

$k \quad$ Maximum cluster size of a group of points in texture. This parameter is used when using a spot or stripe graph traversal strategy for labeling points. 


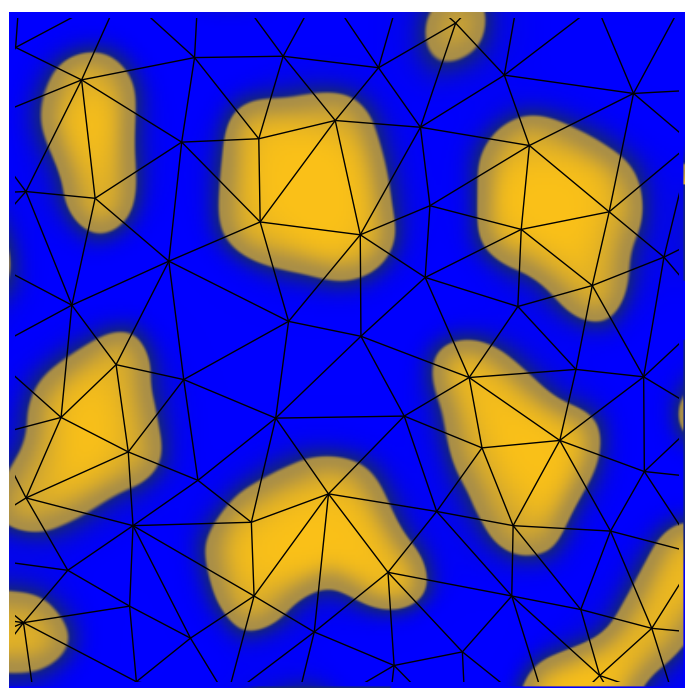

(a)

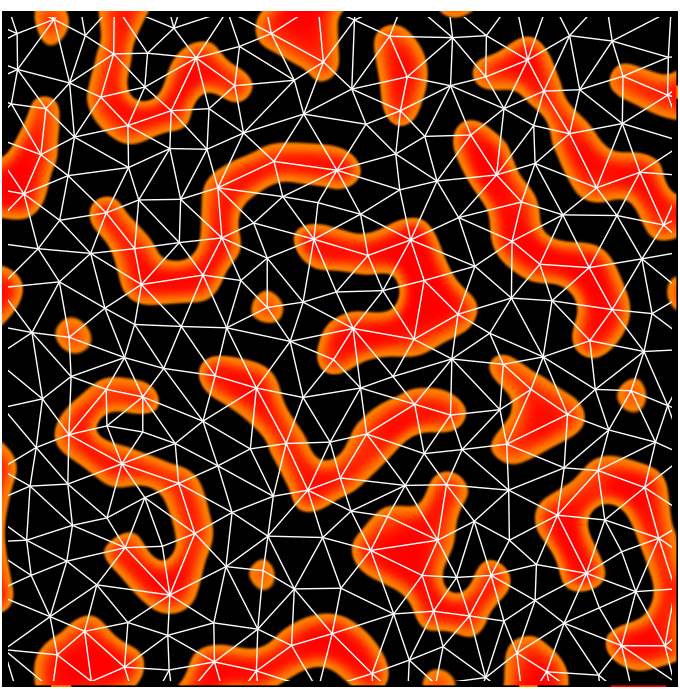

(b)

Figure 5.2: Irregular spot and stripe patterns. (a) irregular spot pattern created with $d=0.10$ and $k=4$ (b) irregular stripe pattern created with $d=0.05$ and $k=8$

\subsection{Irregular Patterns}

Irregular patterns are characterized by regions that vary in both size and shape, and have boundaries that appear uneven and bumpy. We can produce different irregular patterns by using a graph-based labeling strategy, as described in Section 4.4.1.

\subsubsection{Irregular Spot Texture}

We created the spot texture in Figure 5.2(a) as follows. We formed a mesh by distributing points in a Poisson disk distribution with $d=0.10$, followed by applying a spot labeling strategy with $k=4$. We used a color spline for the foreground and background labels, and used a small blending radius to blend the spots with the rest of the texture.

The spots we have created are smooth, but irregularly shaped, resembling the spots found in the spotted marsh frog in Figure 5.1(a). The shape of the spots depend on the underlying mesh, as the edges of the mesh define the region boundaries. Using a Poisson disk distribution with a Delaunay triangulation creates a mesh consisting of triangles which are similar in size and shape, while still providing a source of irregularity for the spots in the texture. 


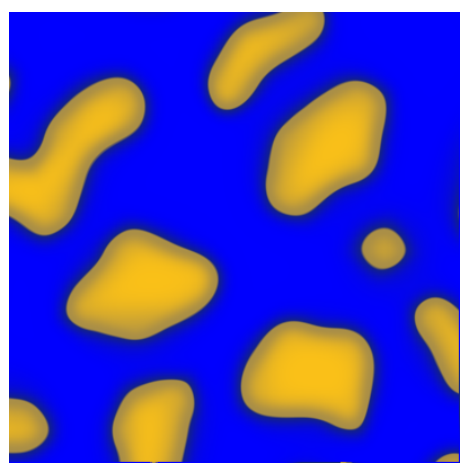

(a) $d=0.10, k=4$

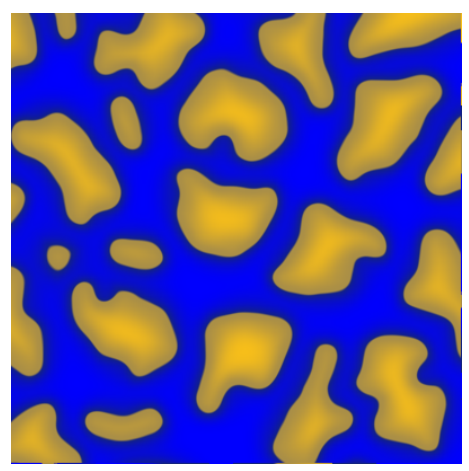

(b) $d=0.05, k=8$

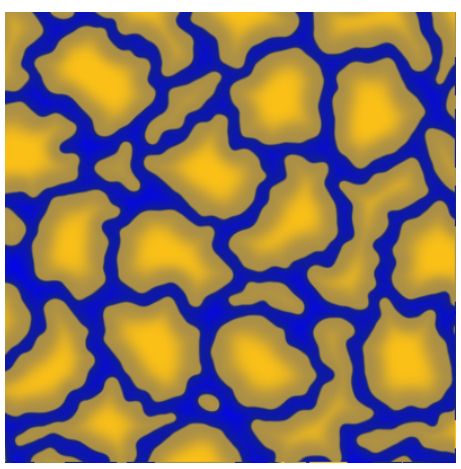

(c) $d=0.025, k=35$

Figure 5.3: Irregular spot patterns each with varying point densities $d$ and cluster sizes $k$.

To create spots that appear more irregular, we can increase the cluster size and point density of the texture. As the number of points belonging to a spot increases, the boundaries of the region become more irregular. This irregularity is caused by the introduction of additional edges used to define the region boundaries, as each introduced edge creates a bend in the distance field of a contour. We show irregular spot textures with increasing cluster sizes and offset distances in Figure 5.3.

Using color splines for the background and foreground labels provide smooth color variations in the texture. Because the distance fields for each spot do not vary widely away from the zero set, we are able to produce a consistent appearance for each spot.

\subsubsection{Irregular Stripe Texture}

We create a stripe texture similar to how the spot texture was created. The irregular stripe texture in Figure 5.2(b) was produced by distributing points in a Poisson disk distribution with $d=0.05$, and then labeled using the stripe labeling strategy of Section 4.4.1 with $k=15$. We show a stripe textures with varying stripe lengths in Figure 5.4. A color spline is used to vary the color in the interior of the stripe.

The procedurally generated texture is intended to be similar to the skin patterns found in the poison dart frog (Figure 5.1(b)). Unfortunately, there are several instances in the texture where a stripe appears rigid and unnatural, such as the bend shown in Figure 5.5. These textures artifacts exist due to how the mesh is defined, 


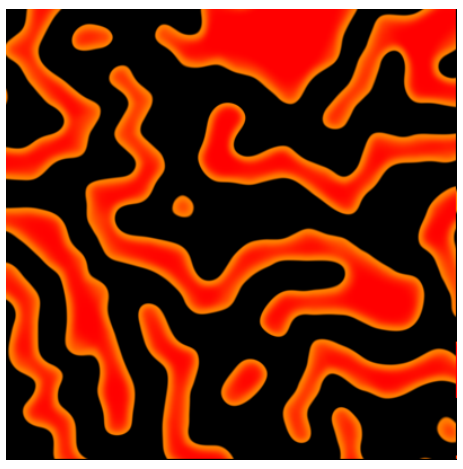

(a) $k=8$

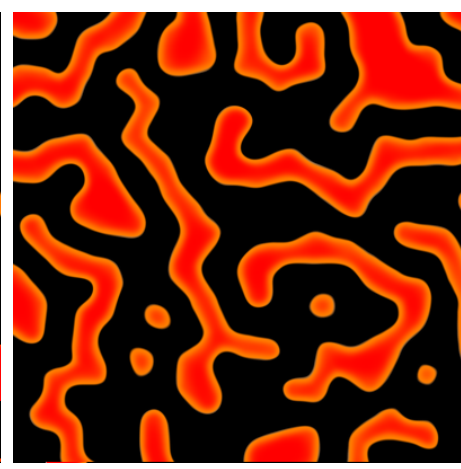

(b) $k=15$

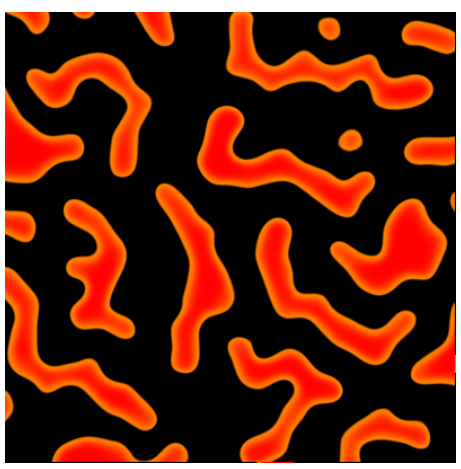

(c) $k=25$

Figure 5.4: Irregular stripe patterns each with varying maximum stripe length $k$.

and occur when two connected edges of a contour form a large bend.

Creating smoother stripes would require modifying the mesh creation process, such as adding additional points along the path of a contour. Section 5.4 .2 will describe how to produce smooth stripes by changing how the mesh is constructed.

\subsection{Smooth Patterns}

In the previous section, we discussed how to produce different irregular patterns, and we gave examples of patterns that resembled certain types of frogs. However, many patterns in nature are more smooth and rounded. This section will describe how smoother spot and stripe patterns can be produced.

\subsubsection{Rounded Spot Texture}

We previously created an irregular spot pattern by grouping neighbouring points together to form a single region. Alternatively, a rounded spot pattern can be created by having each point in the mesh be its own region. An example of such a texture is shown in Figure 5.6(a). The texture was created by distributing points with a minimum spacing of $d=0.10$ in a Poisson disk distribution. Each point is specified with a unique label, and a color spline was used to create an outline for each spot. A pixel that is not within the boundaries of any region is set to be the background color. The spot pattern we have generated shows resemblance to the spotted trunk 


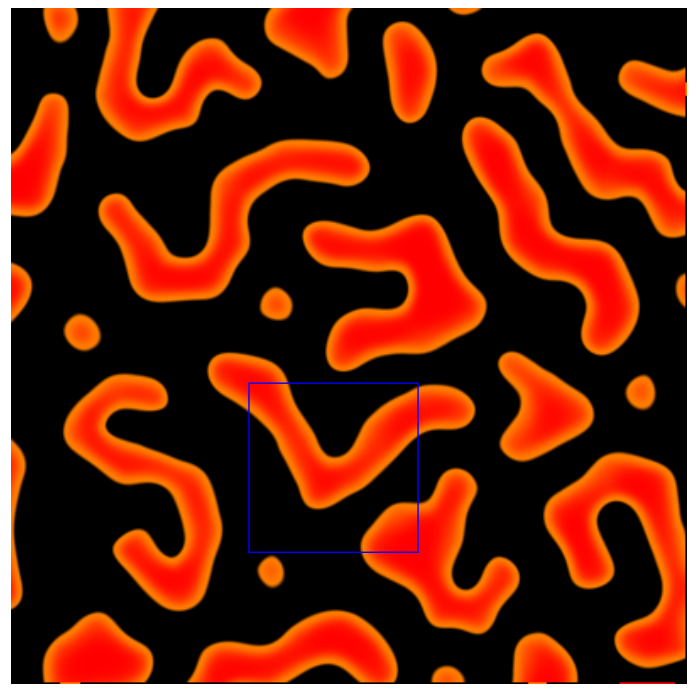

(a)

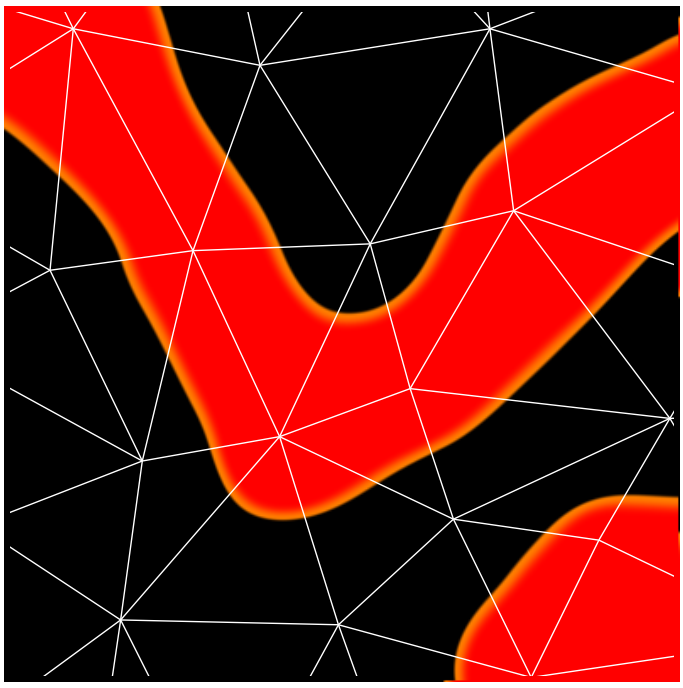

(b)

Figure 5.5: Bend in an irregular stripe pattern. (a) stripe pattern created with $k=8$ and $d=0.10$, and (b) region in the texture with sharp bend.

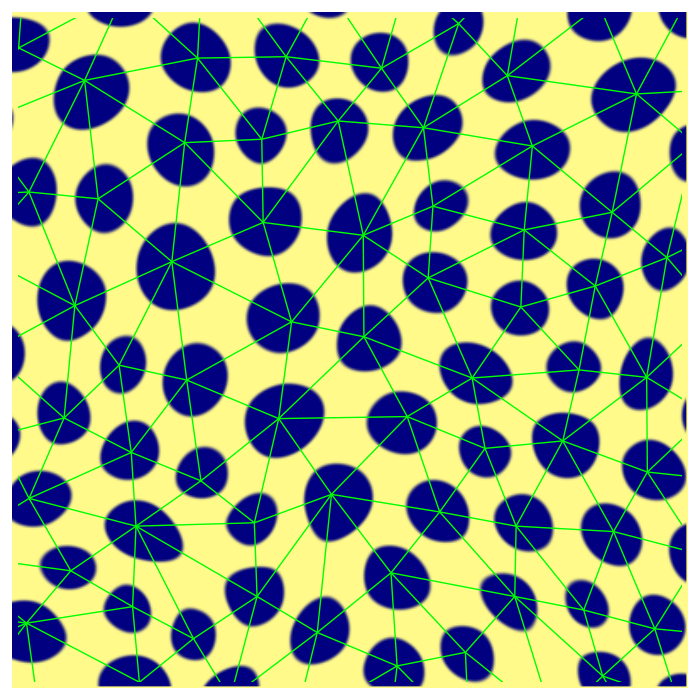

(a)

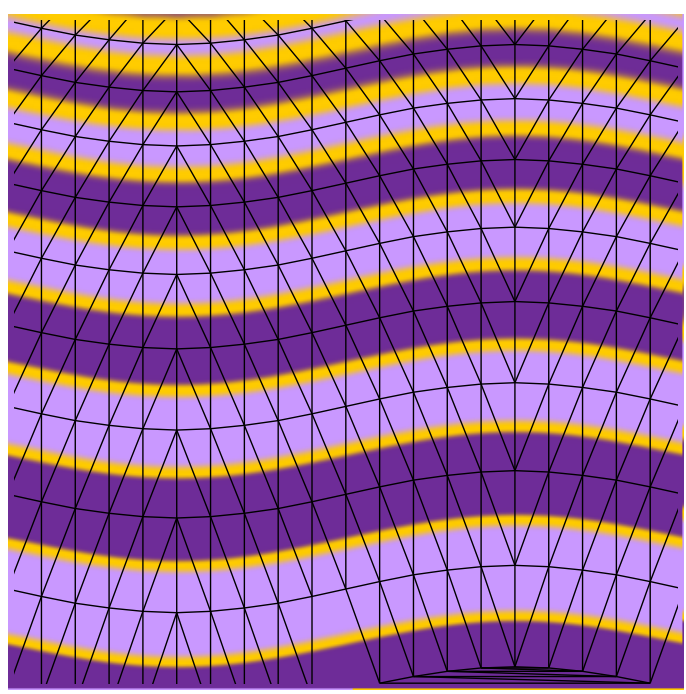

(b)

Figure 5.6: (a) example of a rounded spot texture, and (b) example of smooth stripe pattern. 
fish in Figure 5.1(e).

The behaviour of each spot can be described by the underlying mesh of the texture. The interior contour of a spot consists of a single point, and the exterior contour defines a polygon that connects the neighbours of that point together. Each spot in the texture is a smooth region inscribed within the defined polygon.

\subsubsection{Smooth Stripe Texture}

To create regular stripe patterns, we modify the distribution of points in the texture. By replacing the Poisson disk distribution with other point distributions, we can produce textures with smoother boundaries. Figure 5.6(b) shows an example of a smooth stripe pattern produced by distributing points in a sine wave pattern. Points in the texture are specified by the following set of equations

$$
\begin{aligned}
& x=x^{\prime}+\sin (2 \pi k x) \\
& y=y^{\prime}
\end{aligned}
$$

where $k$ is a parameter used to specify the frequency of the stripe pattern. The variables $x^{\prime}$ and $y^{\prime}$ are points that are sampled from a rectangular grid pattern. For Figure 5.6(b), the grid pattern has a constant horizontal spacing of $w=0.05$ and a variable vertical spacing of $h=0.05+0.01 n$, where $n$ is the index of a particular row of points.

A Delaunay triangulation is used to generate a mesh from the set of points we distributed. Each row of points in the mesh are all given the same label, and a color spline is used to color each individual stripe. The stripe pattern we produced is smooth, and resembles the stripes found on the clown triggerfish, as shown in Figure 5.1(d).

A major limitation of our method is that it is not immediately clear how to produce more complex stripe patterns, such as producing stripe bifurcations. The mesh in Figure 5.6(b) was deliberately generated to produce a particular stripe pattern, and a different labeling of the mesh points would not produce a desirable texture.

A second limitation of our method is evident when we compare between stripes of different thicknesses in Figure 5.6(b): thinner stripes in the texture have a thicker yellow outline. The behaviour of the color spline associated with each stripe is not 


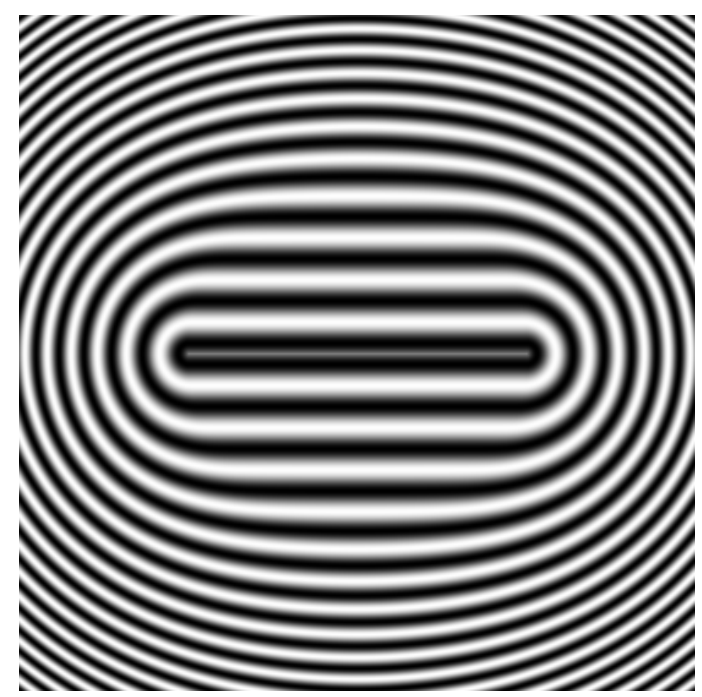

(a)

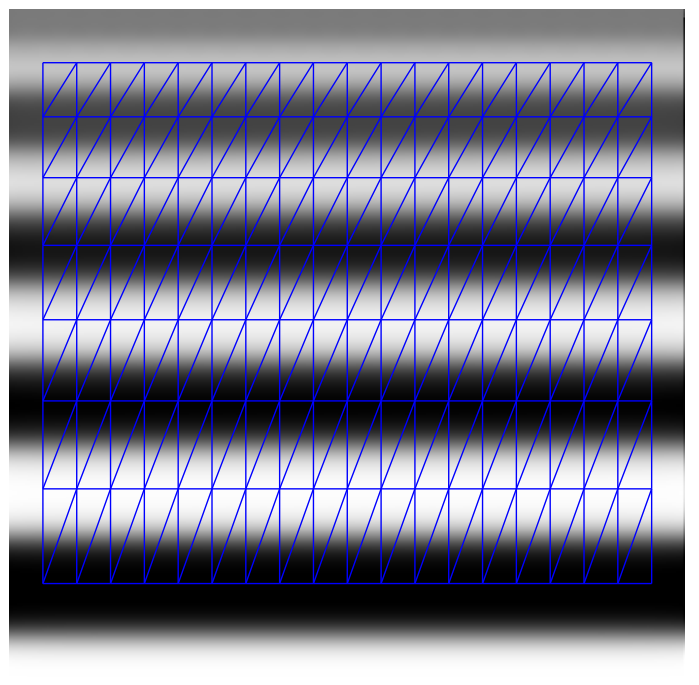

(b)

Figure 5.7: Parameters dependent on smooth distance field. (a) smooth distance field of a single line segment, and (b) implicit field of texture with varying smooth distance fields.

consistent throughout the texture.

To explain the variations in the behaviour of the color spline, we consider the smooth distance field of a single line segment. The isocontours of a field for a single line segment are shown in Figure 5.7(a). We observe that the isocontours of the field are closer together further away from the line segment, which implies that distance values increase faster away from the zero set. This behaviour is a consequence of needing to create a smooth field for the line segment.

The distance field of a stripe in the texture behaves similarly to the distance field of a single line segment, with the gradient magnitude increasing away from the zero set. More generally, the smooth distance field of a region diverges from the exact distance field away from the region boundary. Parameters of our algorithm that depend on the distance field of region, such as the color spline used for the stripe texture, will therefore vary in behaviour depending on the underlying mesh.

Figure 5.7(b) shows another example of a texture parameter being dependent on the underlying mesh. Each stripe was assigned the same blending radius parameter, but the thinner stripes appear to have a much larger actual blending radius. To obtain a uniform blend, a different blending radius parameters would need to be specified for each individual region. 


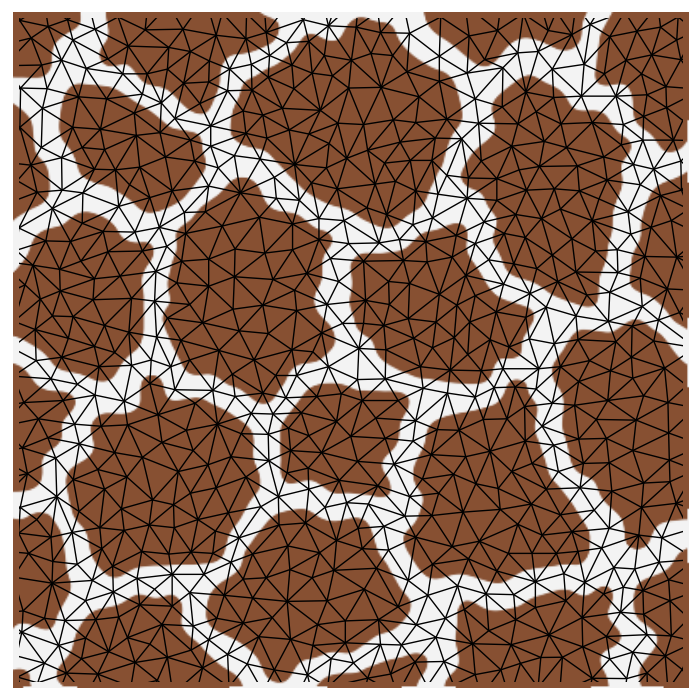

(a)

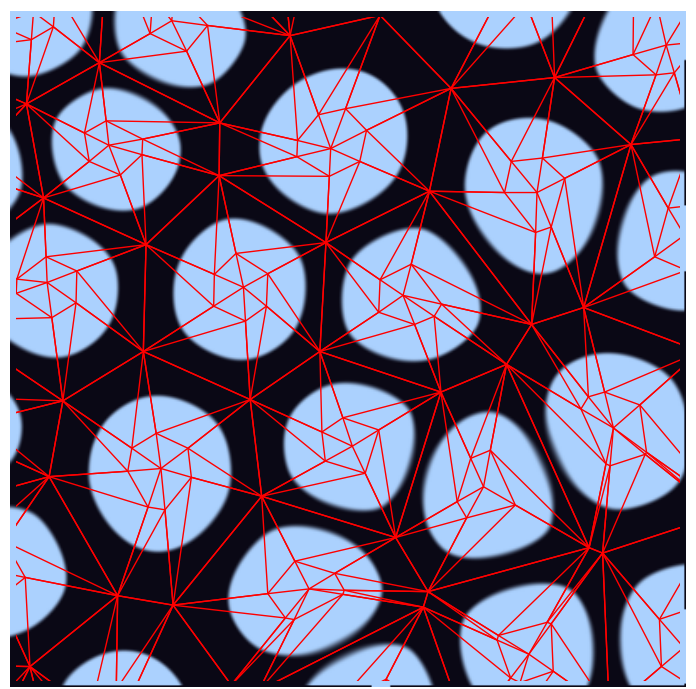

(b)

Figure 5.8: Examples of Voronoi patterns. (a) example of an irregular Voronoi pattern, and (b) example of a smooth Voronoi pattern.

Despite the previously mentioned limitations, we have shown that our method can generate different smooth stripe patterns by distributing points in a systematic way. Stripe patterns similar to Figure 5.6(b) can be found in the emperor angelfish (shown in Figure 5.1(d)).

\subsection{Voronoi Patterns}

We now describe how to produce different cellular patterns based on the construction of the Voronoi diagram. There are many examples of cellular patterns found in the natural world: the spots of a giraffe, the wings of a dragonfly, and the patterns in turtle shells are all examples of Voronoi diagrams found in nature. We will show how to produce both irregular and smooth Voronoi patterns. Examples of both types of patterns are shown in Figure 5.8.

\subsubsection{Voronoi Definition}

We first define several terms related to the Voronoi diagram. A Voronoi diagram is a partitioning of the plane using a set of points. The initial set of points are known as 


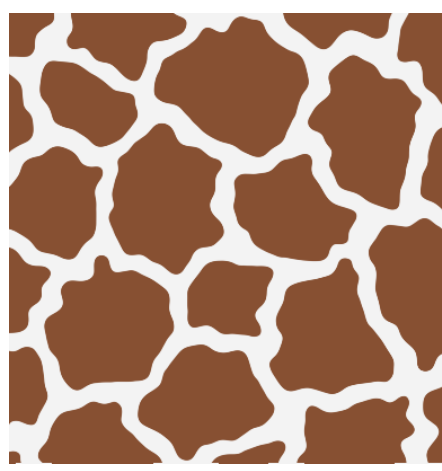

(a) $d=0.030, w=1$

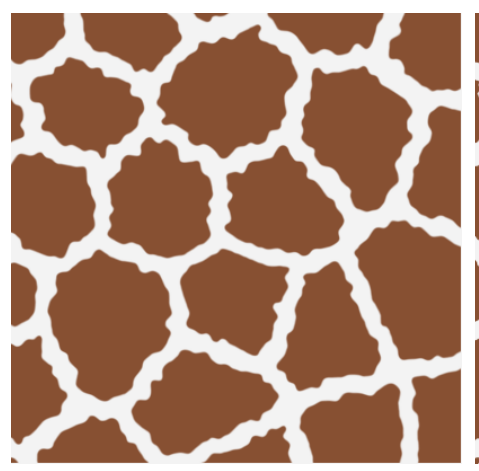

(b) $d=0.015, w=2$

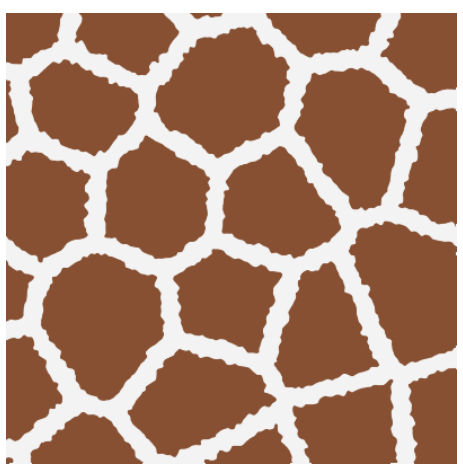

(c) $d=0.0075, w=4$

Figure 5.9: Irregular Voronoi patterns each with varying point densities $d$ and boundary widths $w$.

Voronoi sites, and the partitioned regions are known as Voronoi cells. A Voronoi cell consists of all points that are closer to a fixed site than any other site.

The line segments that form the boundary between Voronoi cells are known as Voronoi edges. A Voronoi vertex is defined as the intersection of three or more Voronoi edges, with each Voronoi vertex being equidistant to three or more sites. We will use the above definitions in the remainder of this chapter.

\subsubsection{Irregular Voronoi Patterns}

Irregular Voronoi patterns are characterized by regions that resemble cells in a Voronoi diagram, but have bumpy and uneven boundaries. An example of such a pattern is found in the common giraffe, shown in Figure 5.1(f).

We can produce a similar giraffe pattern by labeling points on a Voronoi diagram. We distribute points $P=\left\{p_{0}, p_{1} \ldots p_{n}\right\}$ in a Poisson disk distribution, and we distribute a second set of points $S=\left\{s_{0}, s_{1} \ldots s_{n}\right\}$ that are the sites of the Voronoi diagram. All points $p$ that have the same nearest site $s$ are given the same label. All points on the boundary of a group of points are then relabeled to form an outline around each Voronoi cell.

Figure 5.8(a) shows a giraffe pattern created in the above manner, and Figure 5.9 shows irregular Voronoi patterns with different point densities $d$ and boundary widths $w$ between Voronoi cells. 


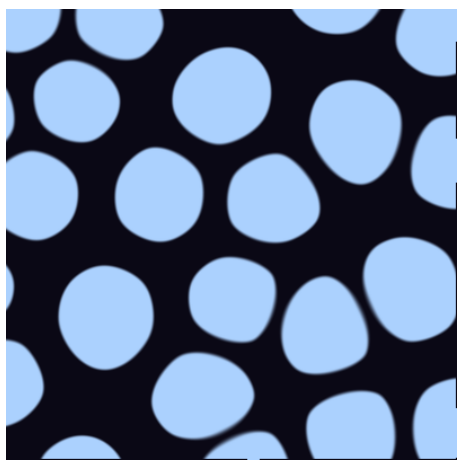

(a)

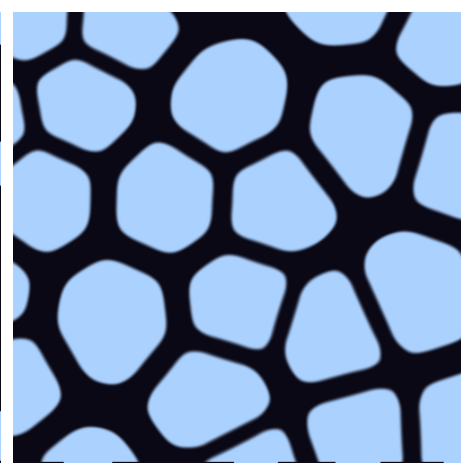

(b)

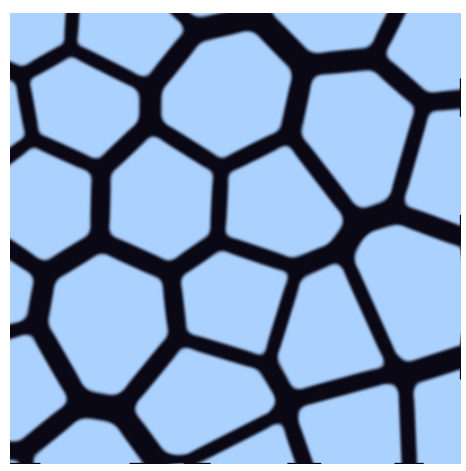

(c)

Figure 5.10: Examples of different smooth Voronoi patterns.

\subsubsection{Smooth Voronoi Patterns}

We now describe how to produce a Voronoi texture with smooth region boundaries. Instead of using a Delaunay triangulation on an initial set of points, we use use the edges of a Voronoi diagram along with an alternative mesh construction method to define region boundaries.

The alternative mesh is constructed as follows. We take the initial set of distributed points $P$ and compute its Voronoi diagram $V(P)$. The vertices of the graph consist of the initially distributed points (which are the sites of the Voronoi diagram), and the set of Voronoi vertices in $V(P)$. The edges of the graph include the Voronoi edges of $V(P)$, and the edges formed by connecting points in $P$ with their respective Voronoi vertices.

A Voronoi texture can be produced by assigning all points in $P$ a foreground label, and all other points the background label. The texture will consist of smooth spots inscribed within Voronoi boundaries. We can control the smoothness of each spot by replacing points in $P$ with offset polygons of the Voronoi boundaries, with an example shown in Figure 5.8(b).

In Section 2.4, we saw how to construct different Voronoi patterns using Worley noise and partition of unity textures. However, those approaches produced fields which were discontinuous at the Voronoi boundaries. This approach is distinguished from the previous approaches by being able to produce a Voronoi pattern that is everywhere smooth, with the smoothness controlled by specifying a particular offset polygon. We show Voronoi patterns with different offset polygons in Figure 5.10. 


\subsection{Complex Patterns}

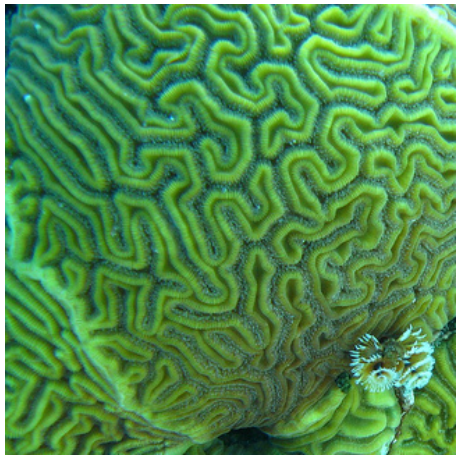

(a)

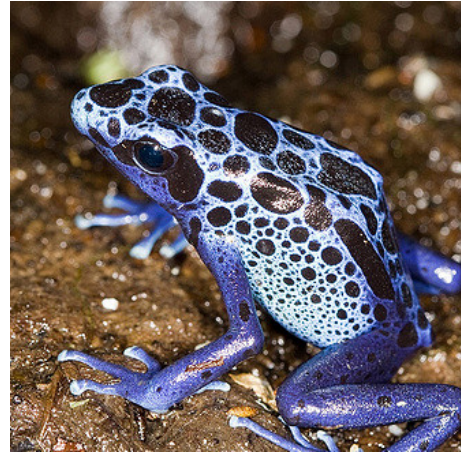

(b)

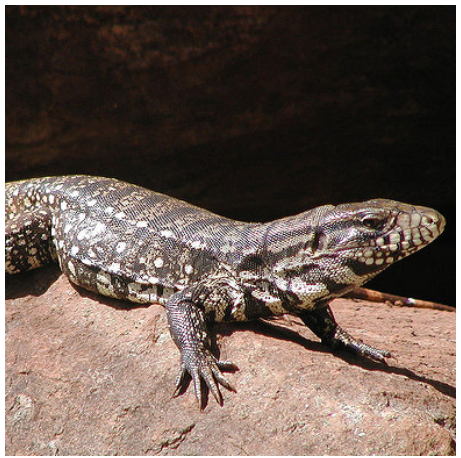

(c)

Figure 5.11: Examples of complex patterns found in nature: (a) maze coral [10], (b) blue poison arrow frog [40], and (c) lizard [7]

We now describe approaches to obtain more complex textures. Examples of the complex textures we wish to produce are shown in Figure 5.11. We will show how to use a secondary image to guide point labeling, how to produce different non-stationary textures, and how boundary constraints can be added to a texture.

\subsubsection{Image Texture}

One way of obtaining more complex patterns is to change the how points are labeled. Rather than use a graph traversal method (Section 4.4.1), we use a binary image to define whether a point is assigned a background or foreground label. For a binary image $B(p)$ the maps every location $p$ to either 0 or 1 , a point is assigned the foreground label if $B(p)=1$, and is assigned the background label otherwise. Figure 5.12 shows a complex pattern created using a procedurally generated maze image.

Instead of using a binary image, we can use a grayscale image to give a probabilistic assignment of point labels. We let $F(p)$ be a grayscale image such that $0 \leq F(p) \leq 1$, and we let $\alpha$ be a continuous uniform variable in the range $[0,1]$.

A point $p$ is assigned the foreground label if $F(p)>\alpha$, and is assigned the background label otherwise. Figure 5.13 shows several examples of textures created using 


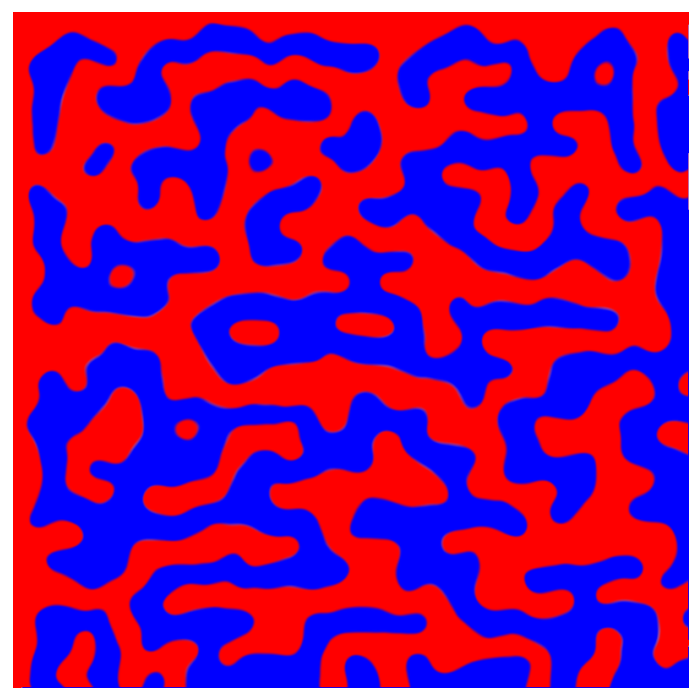

(a)

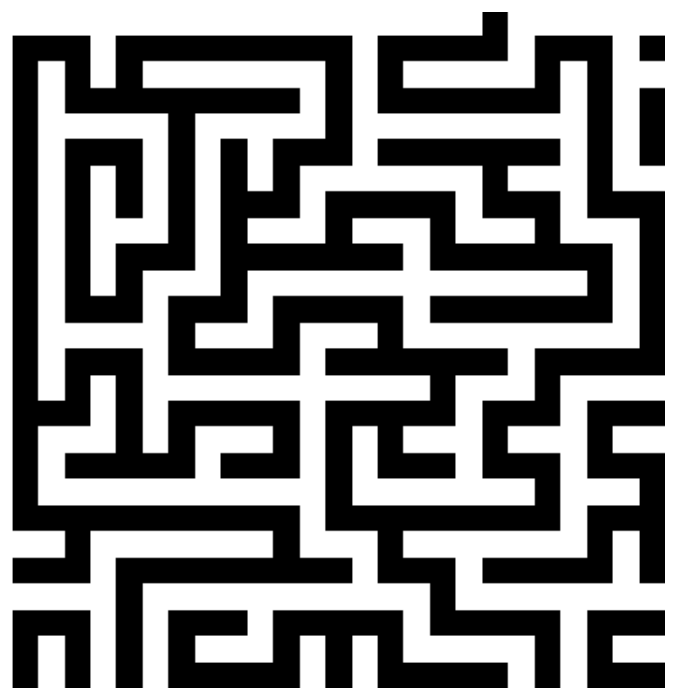

(b)

Figure 5.12: Example of maze texture. (a) maze texture, and (b) corresponding binary image.

a probabilistic assignment of labels from an image. The binary textures consist of smooth regions but are non-stationary, and resemble the supplied image.

\subsubsection{Non-Stationary Texture}

We now describe how to produce different non-stationary textures that vary the size and shape of the texture elements across the image. Many examples of non-stationary patterns can be found in the natural world. One such example is the blue poison arrow frog, which is shown in Figure 5.11(b).

One approach to creating non-stationary texture is to vary the point distribution across the domain, as described in Section 4.5. The texture shown in Figure 5.14 was produced with a variable density point distribution, with spot sizes being larger or smaller depending on the local point density.

Another approach to creating non-stationary texture is to vary how points are labeled. Figure 5.15 shows a texture produced by varying the cluster size of the different spots. We have used a grayscale image to determine the spot size, with areas of higher intensity in the image corresponding to larger spot sizes in the texture. 


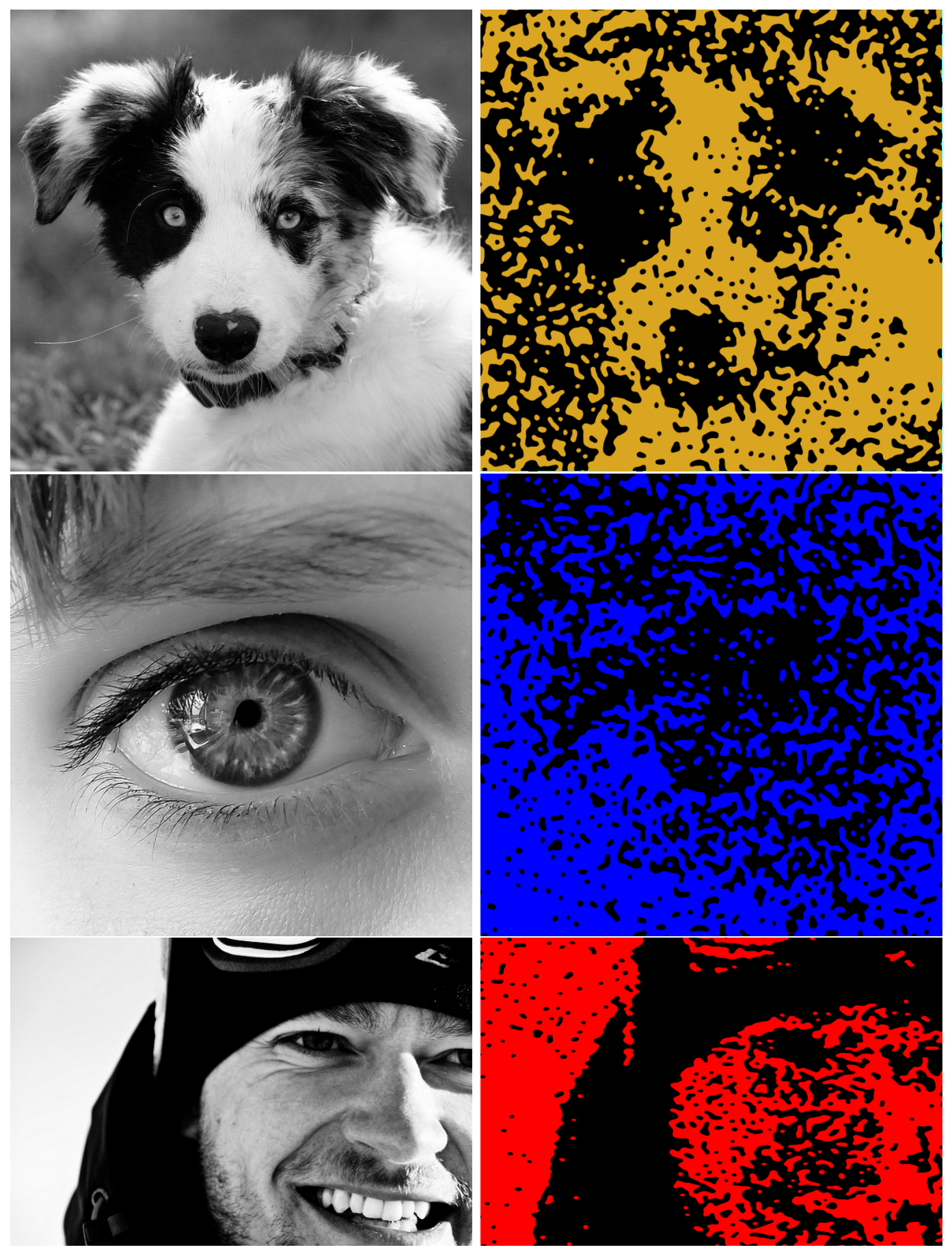

Figure 5.13: Examples of textures created from images $[5,45,67]$. (a) original image, and (b) texture produced using probabilistic assignment of labels from image. 


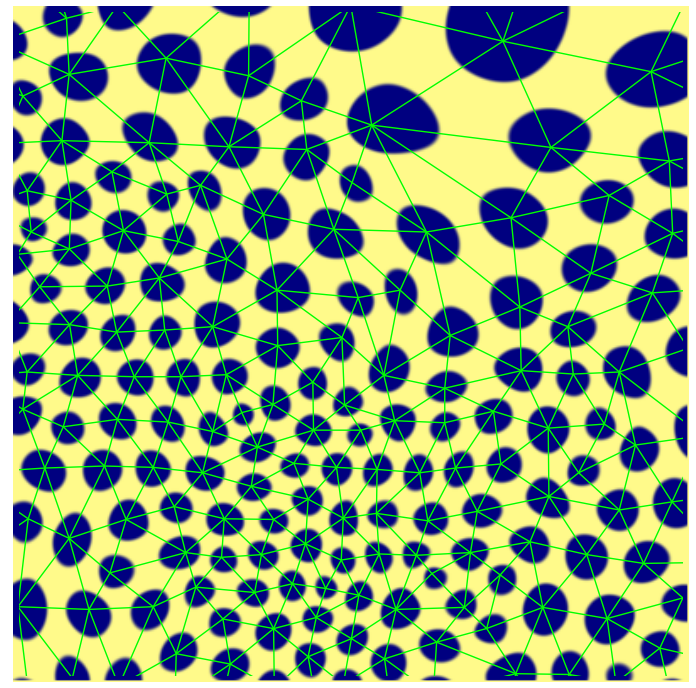

(a)

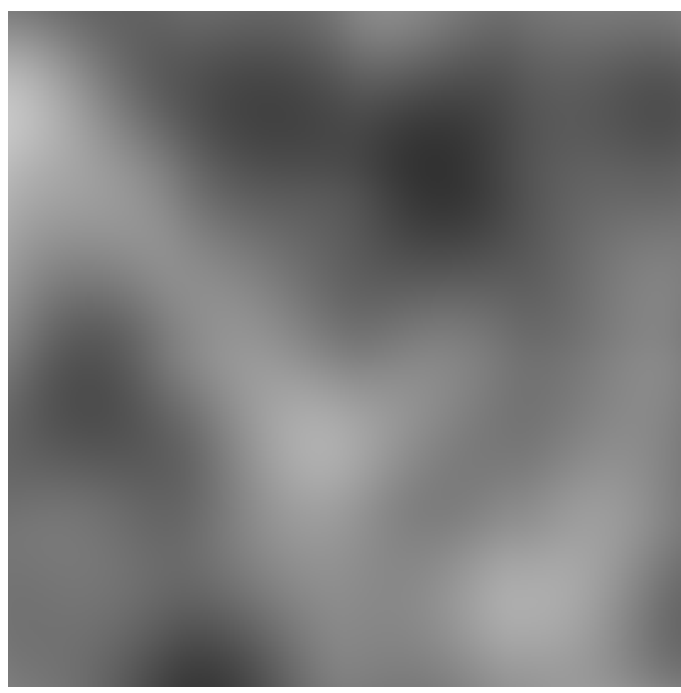

(b)

Figure 5.14: Example of non-stationary texture. (a) non-stationary spot texture, and (b) corresponding density map.

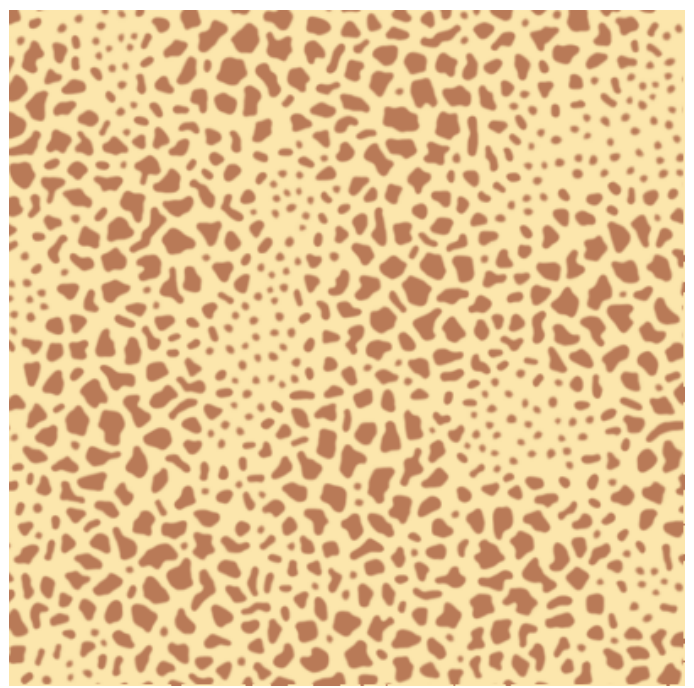

(a)

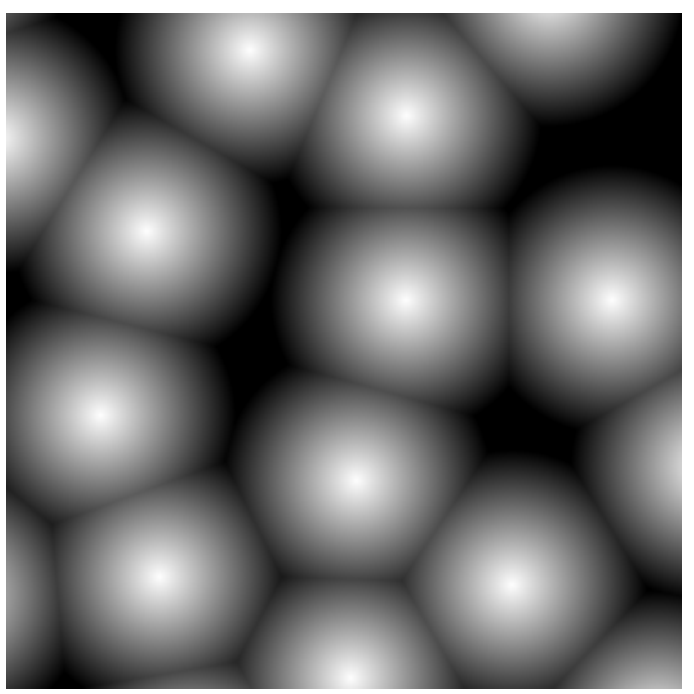

(b)

Figure 5.15: Example of non-stationary labeling. (a) texture with varying cluster sizes for each spot, and (b) image used to determine cluster size. 


\subsubsection{Boundary Constraints}

We have shown how to produce several different types of irregular patterns by distributing points in a Poisson disk distribution, and then forming a mesh using a Delaunay triangulation. The boundaries of those patterns were irregular because of how mesh edges were used to define region boundaries, but we can construct smoother regions by directly modifying the underlying mesh of the texture.

Figure 5.16 shows a random binary texture constrained to be within a circular region. Points were added to the boundary of the region, so that no spot would extend beyond that boundary. By adding a second set of boundary points, shown in Figure 5.17, we ensure spots near the boundary are curved to follow the circular outline. Manipulation of the underlying mesh allows us to create both smooth and irregular regions in the texture.

\subsection{Summary}

In this chapter, we discussed how different textures can be created by varying the parameters of our algorithm. We showed how to create different irregular stripe and spot textures by using a color spline with a graph-based labeling strategy. We then showed how to create smooth patterns and Voronoi patterns by changing the point labeling, point distribution, and graph construction of our method. Lastly, we described several techniques to create more complex patterns. 


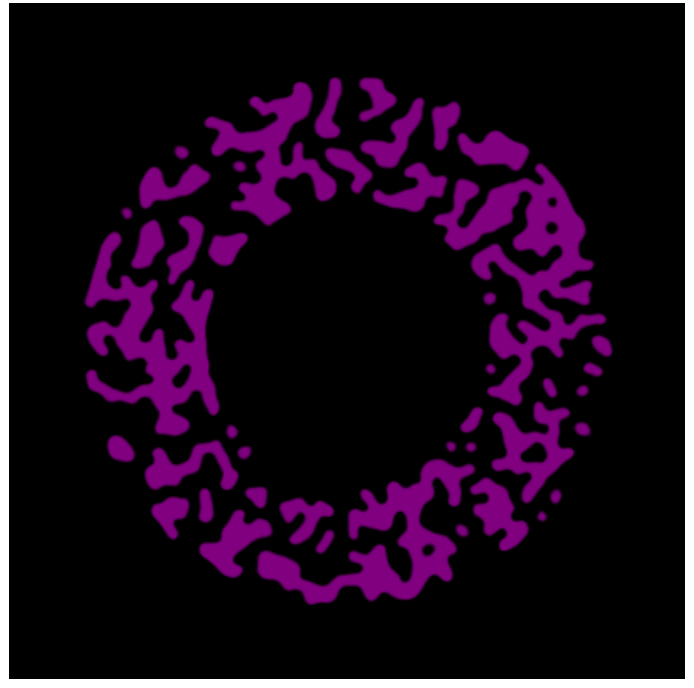

(a)

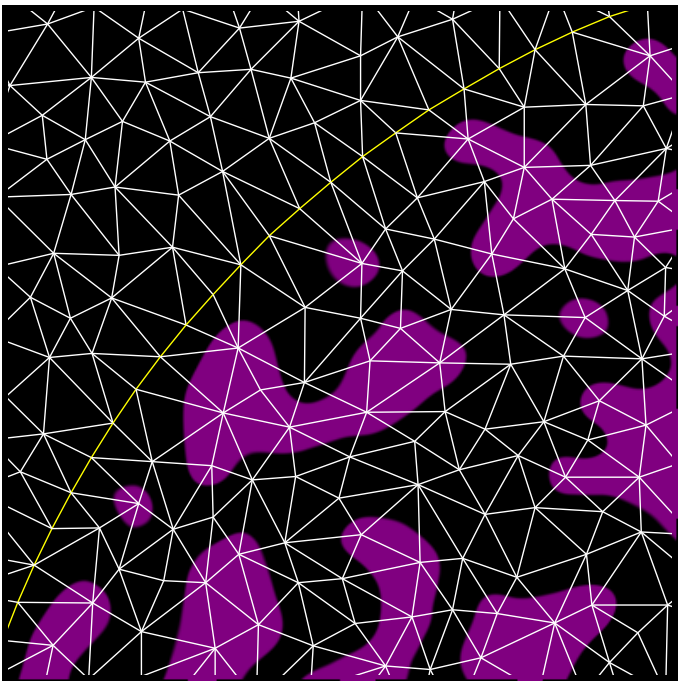

(b)

Figure 5.16: Texture with single set of boundary points. (a) complete texture, and (b) section of the texture, with boundary edges shown in yellow.

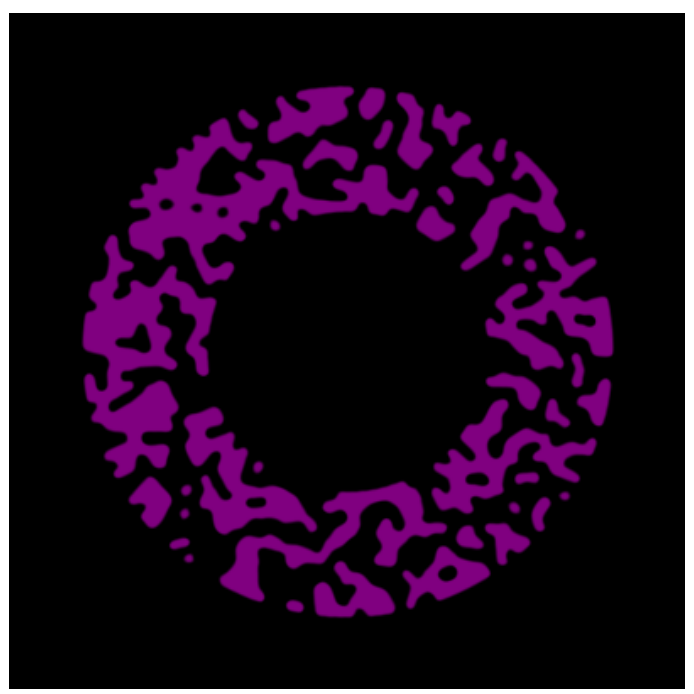

(a)

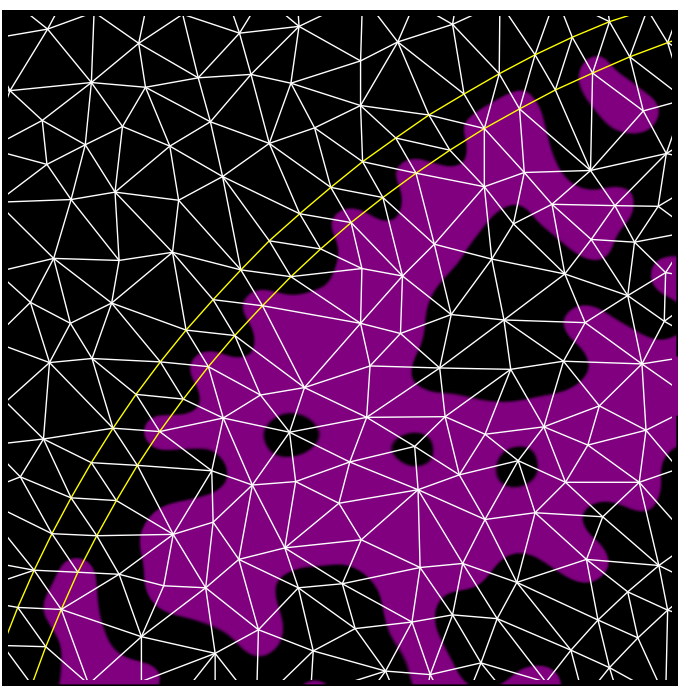

(b)

Figure 5.17: Texture with two sets of boundary points. (a) complete texture, and (b) section of the texture, with boundary edges shown in yellow. 


\section{Chapter 6}

\section{Discussion}

\subsection{Rationale}

We started with the goal of creating smooth organic patterns, and we wanted these patterns to be created procedurally with a minimal set of parameters. The user would not be required to specify individual elements of the texture, such as spline control points. The solution we presented uses smooth signed distance fields to define an organic texture. In this section, we discuss the rationale, benefits, and limitations of our approach.

\subsubsection{Mesh Texture}

We chose to use the points of a mesh to define our texture, with the edges of the mesh defining the boundaries of regions in our texture. We used a mesh for the simplicity of the texture definition: a suitable mesh is constructed by distributing points in the plane, and triangulating. We can exploit many existing methods for both point distribution and mesh creation. For example, Section 5.6.2 described how to use a mesh with a varying point density to create non-stationary texture.

Another benefit of using a mesh is that the mesh provides a natural way to group points together, and provides a way to form regions from the grouped points. Specifically, points are grouped together if they share the same label and are connected to one another in the mesh, and the boundary for a group of points is formed from neighboring edges. We also use the mesh for point labeling; Section 4.4 described several different methods of reasoning over a graph. By having a mesh we were able to create various kinds of spot and stripe patterns. Furthermore, using a mesh avoids 
the lattice artifacts present in other techniques such as Perlin noise.

\subsubsection{Field Definition}

After points of the mesh are grouped together, we defined for each point group the interior and exterior contour. We computed a smooth signed distance field for each contour, and blended the distance fields together to create a signed distance field for a region.

The rationale for using a pair of smooth distance fields, rather than the distance field of a single contour, was to create a combined field that was smooth everywhere. As described in Section 4.1.5, wherever one distance field was discontinuous, we would use the distance field of the opposing contour.

In addition to producing an everywhere smooth field, using a pair of distance fields limits bulging in the distance field of the region. Bulging of a smooth distance field occurs when two line segments are joined together using the R-equivalence operation. Figure 6.1(a) shows the isocontours of a smooth distance field from a single line segment. Figure 6.1(b) shows, with a bulge present, a smooth distance field of two line segments joined using R-equivalence.

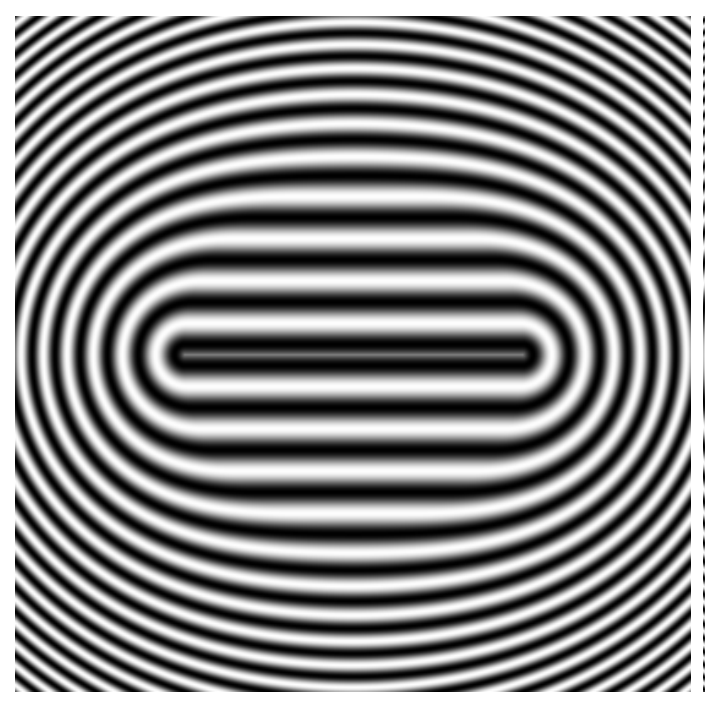

(a)

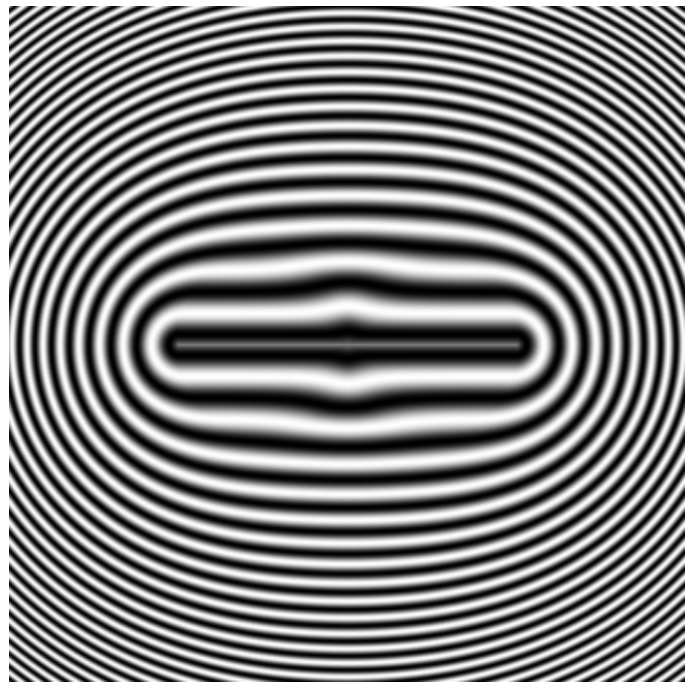

(b)

Figure 6.1: Bulging in a smooth distance field. (a) Single line segment smooth distance field. (b) Bulge formed by joining two separate line segments.

Biswas and Shapiro [11] provided an analysis of the problem based on the gradient 
magnitude at the zero isocontour. Recall that in the case of an exact distance field, for any point not on the medial axis, the gradient magnitude is unity and all higher derivatives vanish.

In the case of the smooth distance field, the gradient magnitude is not unity everywhere, and specifically is less than unity at a joining point where two line segments meet. Figure 6.2(a) shows the field of two line segments joined at 90 degrees. Figure 6.2(b) shows regions, marked in gray, where the gradient magnitude is less than 1. For a R-equivalence operation of two line segments, a further analysis [74] shows that the minimum value of the gradient occurs at the bisector of the joined segments.

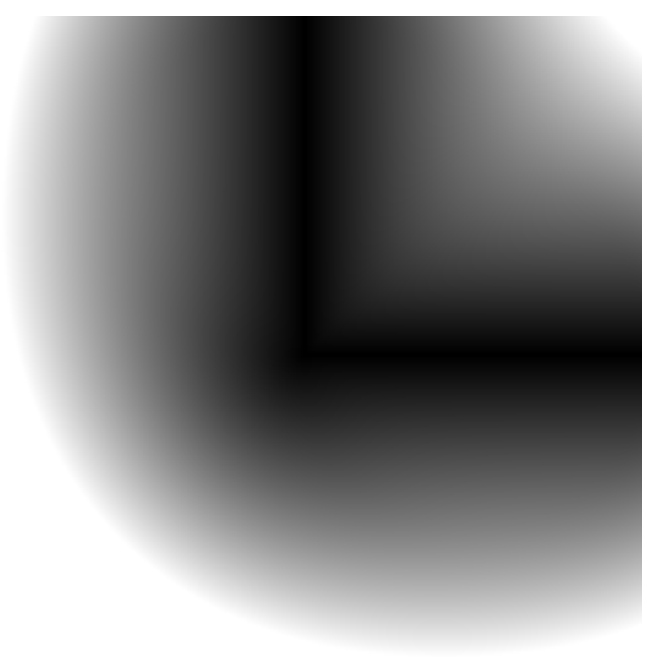

(a)

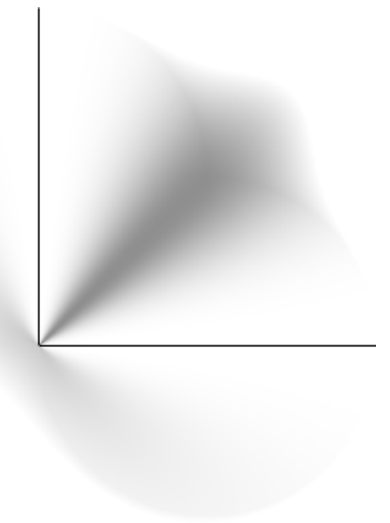

(b)

Figure 6.2: Analysis of bulging problem. (a) Implicit field of two joined line segments. (b) Gradient magnitude of joined line segments.

This bulge problem has been discussed in the context of other methods. In the case of binary blending, Gourmel [33] proposed the solution of blending two implicit primitives based on the angle between their respective gradient fields. An improved method for general composition was later considered by Canezin et al. [16]. These methods produce smooth bulge-free joins between implicit primitives, but have the limitation of not being associative: the field depends on the blending order of the primitives.

In the case of implicit modeling, convolution surfaces [13] were proposed as a generalization of point potential surfaces to reduce bulging. In this method, the field 
of a primitive (such as a line segment or polygon) is calculated as the convolution over the primitive with a specific kernel function. A choice of an appropriate kernel can then reduce visible bulges, although the field itself will not be bulge-free. Variational implicit surfaces [85] use radial basis functions to define constraints to form bulge free surfaces. The fields produced with radial basis functions are smooth everywhere, but are generally not well behaved away from the zero set.

In our method, bulging from a single contour is limited because we blend between two smooth distance fields. The weights used in blending a distance field increase as the distance grows. Since bulges are most prominent at the zero isovalue of a smooth distance field, the bulge that is present in one contour is replaced by the distance field of the opposing contour.

If the interior and exterior contours run parallel to each other, the result is a near bulge-free field. Figure 6.3 shows a smooth field produced using an interior and exterior contour, while Figure 6.4 shows a field produced using only a single set of line segments. Bulging occurs at the corners of Figure 6.4, but is not evident in Figure 6.3.

When the interior and exterior contours are not parallel, field irregularities may occur from the interaction between the two distance fields. Figure 6.5 shows a field that is produced when contours are not aligned. The combined field is more complex, because each contour introduces a different bend into the distance field.

The mesh definition is therefore important in controlling irregularities in the tex-

ture. While this is a significant drawback, our method visible reduces bulging for the entire implicit field, rather than only at a particular isovalue. Furthermore, unlike the method of Gourmel [33], our method does not depend on the blending order of line segments.

\subsubsection{Texture Synthesis}

The implicit fields of each region are combined to generate a texture. In Section 4.1.6, we showed how to produce a simple binary texture by assigning all pixels in foreground regions a single color, and assigning a different color to the remaining pixels.

Instead of defining a region using an implicit field, we could have defined a region by only specifying a boundary. For example, we could have defined a closed spline curve over a particular group of points in the mesh. The texture would be created by 


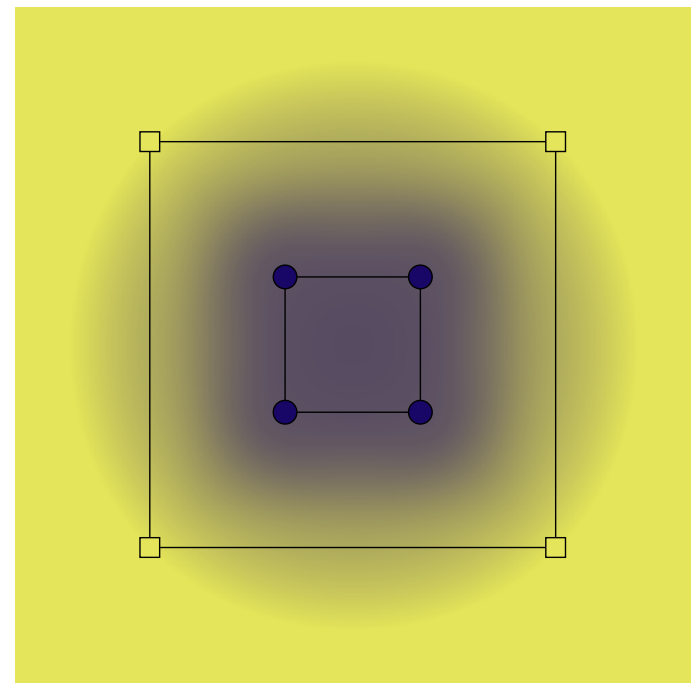

(a)

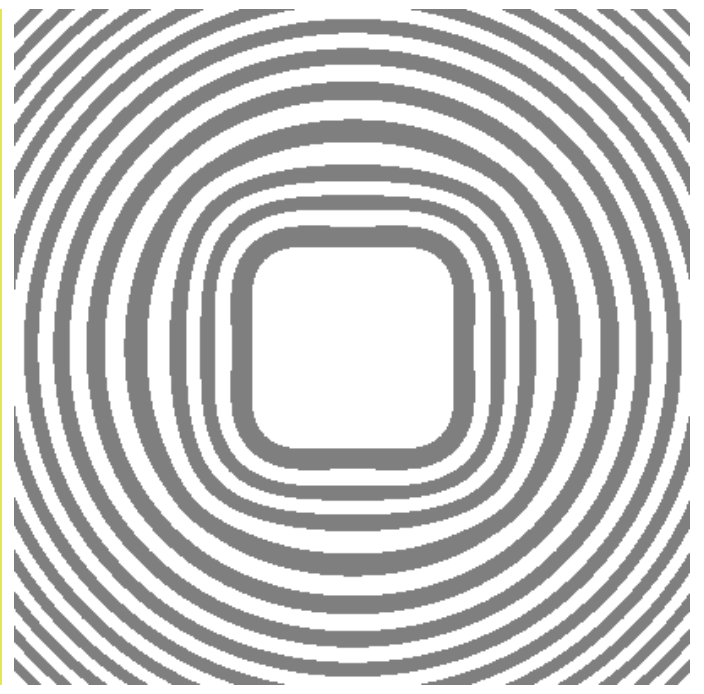

(b)

Figure 6.3: Implicit field created using an interior and exterior contour. (a) implicit texture, and (b) isocontours of the produced field.

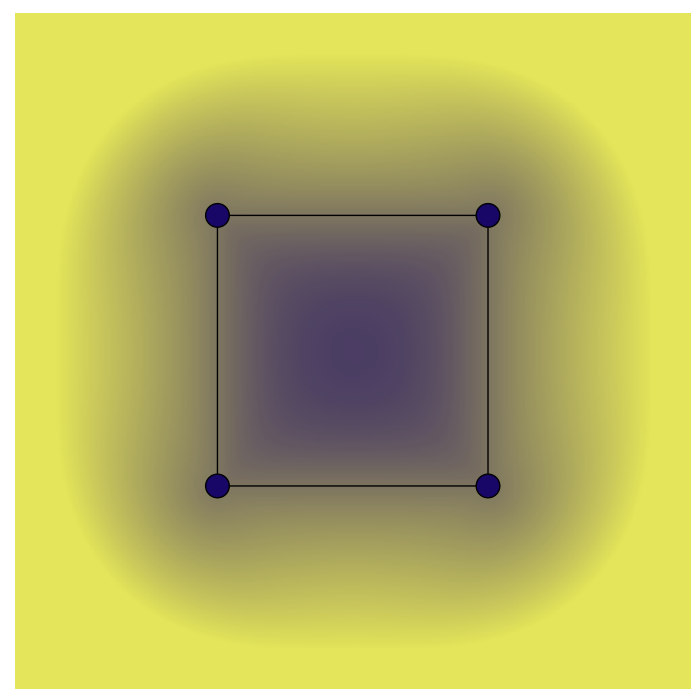

(a)

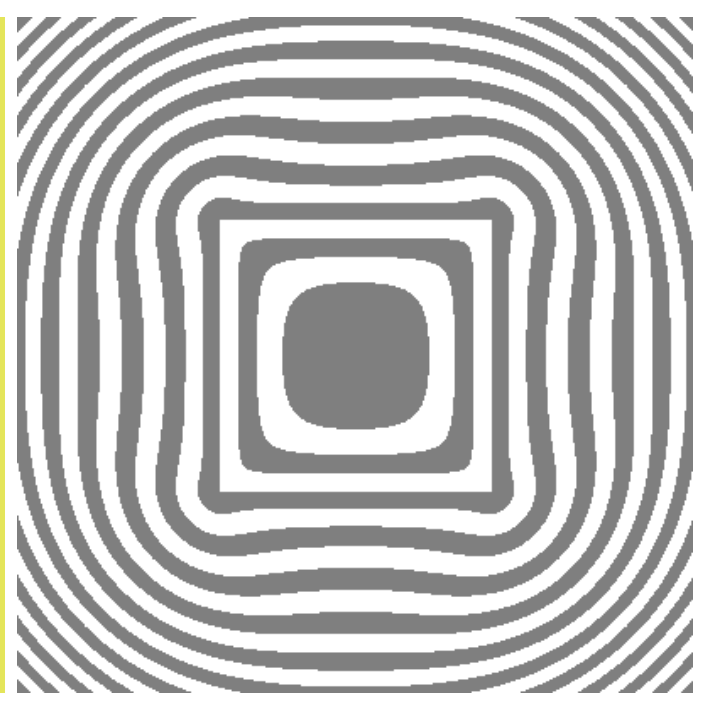

(b)

Figure 6.4: Implicit field created using a single set of line segments. (a) implicit texture, and (b) isocontours of the produced field. 


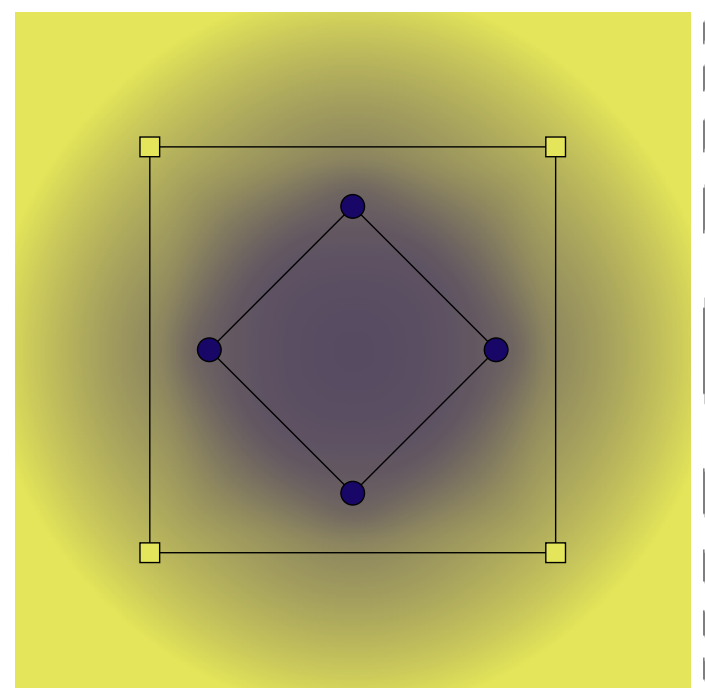

(a)

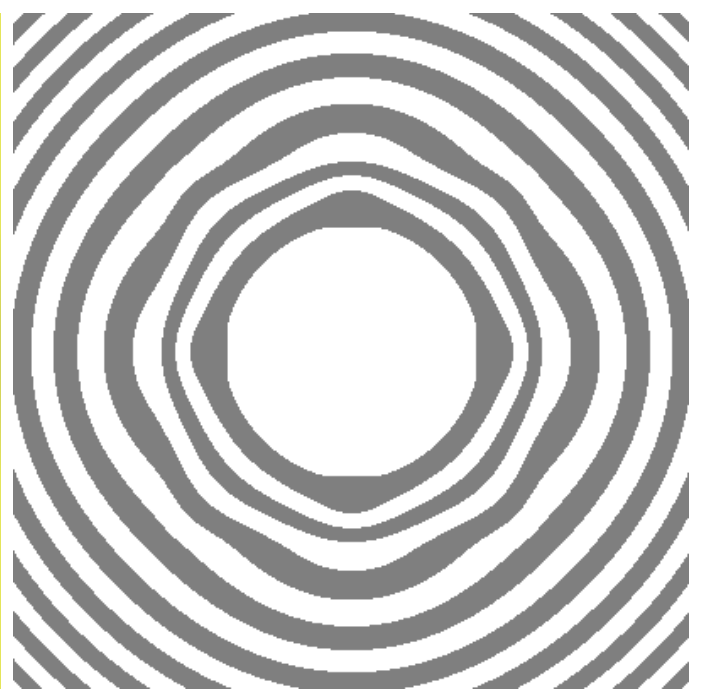

(b)

Figure 6.5: Texture with rotated interior contour. (a) implicit texture, and (b) isocontours of the produced field.

determining whether a point was inside or outside the defined curve.

The benefit of using an implicit field is that we are able to vary the behaviour within a region in the texture. Specifically, having an implicit definition of the texture allows us to blend regions together, and allows for the color of a region to vary in its interior. For textures created with a binary labeling, there is a natural symmetry in the field definition: the interior contour of one region is the exterior contour of another region. The generated fields of each region will therefore blend smoothly with one another.

Because of how distance fields are used, our approach requires that field values do not vary widely away from the region boundary. We have chosen to use R-functions to produce the distance fields of each contour of a region.

Chapter 5 showed several examples of using the smooth distance field of each region to create more complex textures. The textures we produced are both smooth and continuous, and can contain different non-convex shapes.

\subsubsection{Comparison}

Chapter 2 previously mentioned partition of unity textures and reaction-diffusion textures as other methods that can be used to produce different types of smooth 
organic texture. Our method is distinguished from previous approaches by being able to generate texture that is continuous, and that can contain smooth and non-convex texture elements. This section gives a comparison between our method, Worley noise, and partition of unity textures.

The approach we take is similar to partition of unity textures in several ways: we use a mesh to define our texture, and we use a weighted average of field values to determine the color of a pixel. A major drawback of partition of unity textures is the difficulty in creating smooth non-convex shapes. To see this drawback, we observe that the weight functions possess the Kronecker delta property (Section 3.3.3), and will therefore have a texture with unit gradient at each feature point. When a region is comprised of multiple points, the boundary of the region becomes bumpy and irregular. Figure 6.6 shows several textures generated using partition of unity. In our method, the smoothness of a boundary is governed by the edges of the mesh. We are able to produce both irregular and smooth patterns by varying how a mesh is defined and constructed, as shown in Figure 6.8.

Reaction-diffusion textures use chemical simulation systems to generate a variety of organic patterns, including the smooth and non-convex regions we desire. Reactiondiffusion textures can also generate additional patterns that cannot be easily produced with our method. For example, leopard spots were created using reaction-diffusion by using a cascade system of chemical reactions, as described in Section 2.5. We show several patterns generated with reaction-diffusion in Figure 6.7.

The difficulty with using reaction-diffusion textures is that the method requires numerically solving a set of non-linear partial differential equations. The texture is discretized to a particular resolution, and the parameters of the texture may be difficult to control. In contrast, our approach gives a continuous texture representation, with region boundaries defined using the mesh edges. The parameters of our method are more closely related to the final output texture, and we expect to create different textures by varying how a mesh is defined and labeled.

\subsection{Storage and Performance}

In addition to producing smooth organic textures, we also desire our procedural texture function to use as little memory as possible, and to be able to quickly generate a texture. This section describes the storage and computational complexity of our 


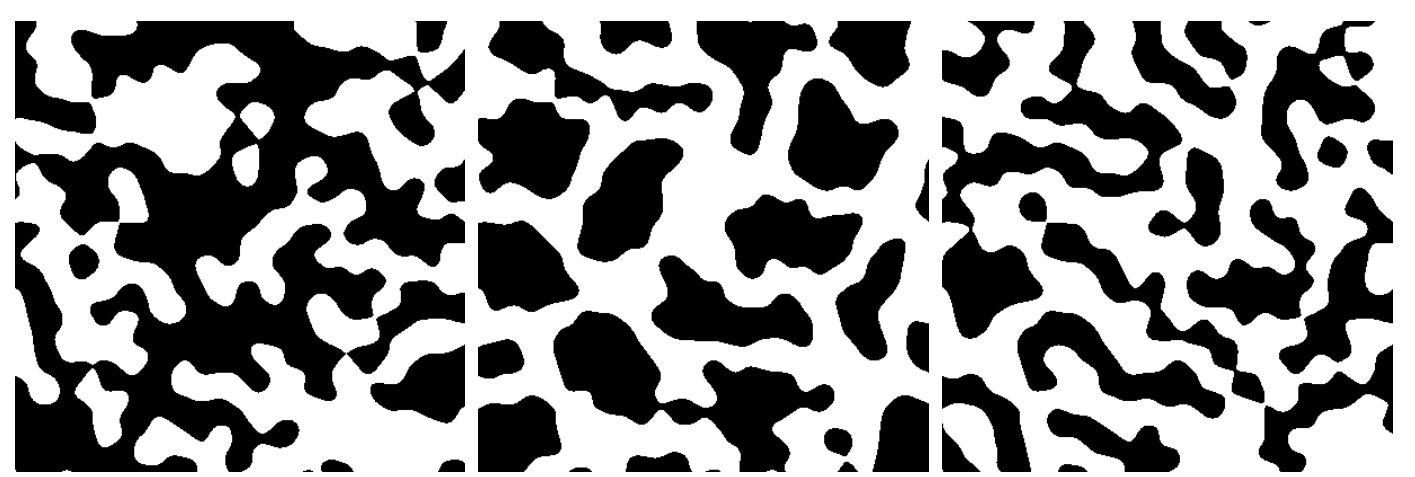

(a) Random binary

(b) Breadth-first

(c) Depth-first

Figure 6.6: Examples of patterns created using partition of unity textures.

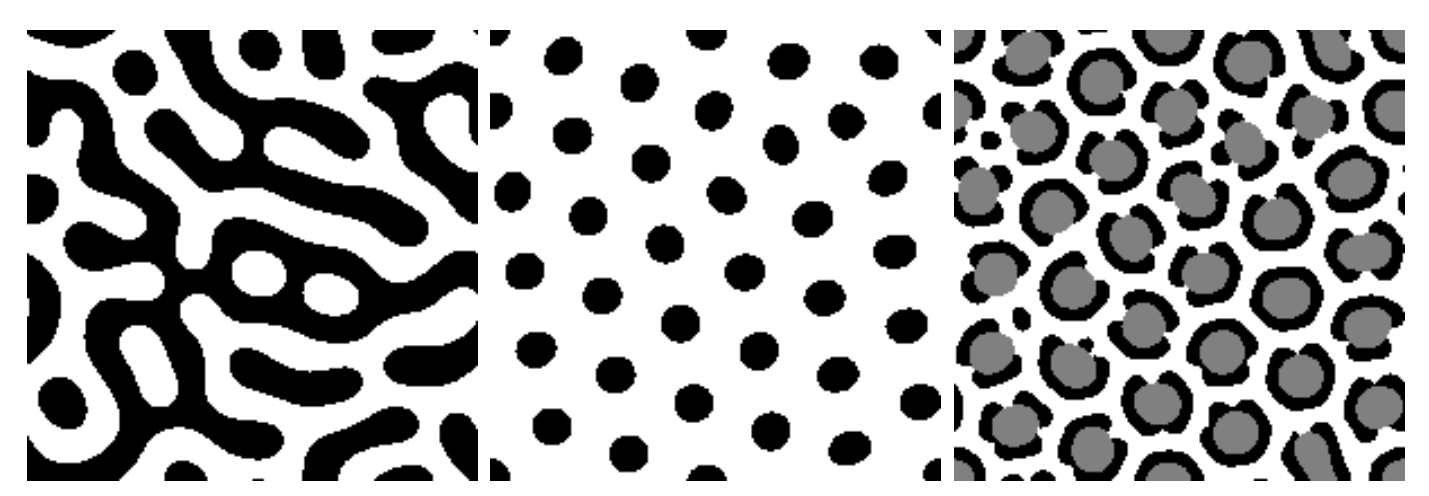

(a) Stripe pattern

(b) Spot pattern

(c) Leopard pattern

Figure 6.7: Examples of patterns created using reaction diffusion.

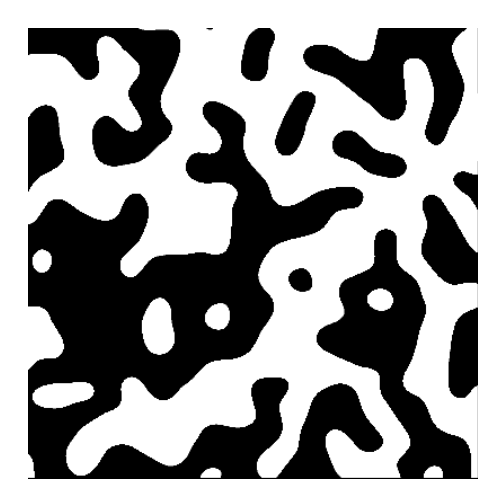

(a) Random binary

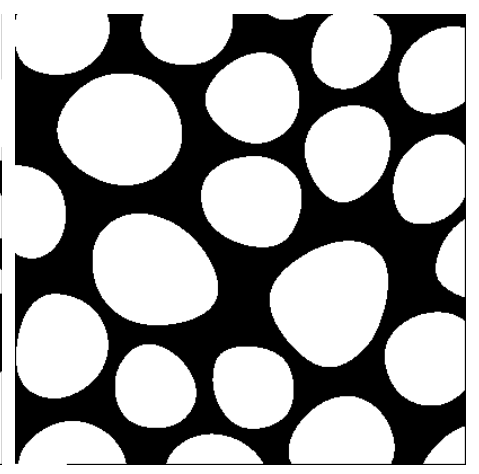

(b) Spot pattern

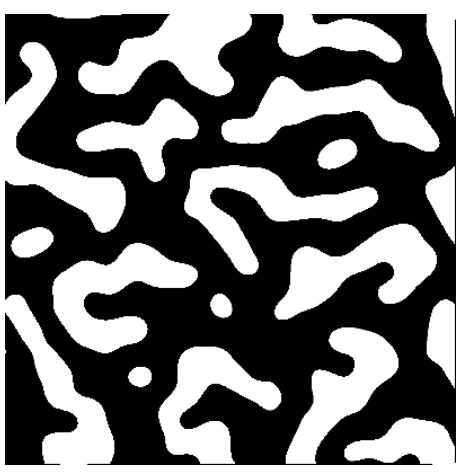

(c) Stripe pattern

Figure 6.8: Examples of patterns created using our method. 
algorithm.

Because our texture is defined using a mesh, storage of our texture is linear in the number of mesh vertices. The entire mesh, as well as the labels associated with each point, are required to determine the pixel value at a specific location.

\subsubsection{Random Access}

To evaluate the texture at a location, we first find a set of candidate regions that may contribute to the pixel value. We determine the set of candidate regions as follows. For each region, we determine the maximal area in the texture that the region can contribute to the pixel value, and this area we refer to as the support of the region. We form a boundary box around each region based on its support, and place the regions into a spatial partitioning structure. The set of candidate regions can be obtained in $O(\log n)$ time in the number of regions.

Once we have obtained the set of candidate regions, we evaluate the contribution of each region to determine the pixel value. The field value of all regions are combined together to determine the pixel value. Because we use a spatial partitioning structure to determine the set of candidate regions, random access evaluation of the texture is dependent on the number of regions in the texture.

\subsubsection{Fixed Resolution}

Instead of determining the value of the texture one pixel at a time, a faster method can be used to obtain the entire texture at once for a given $n \times n$ resolution. Instead of using a spatial partitioning structure to determine which regions contribute to a pixel, we iterate through the support of each region and add the region contribution to the corresponding pixel. Such an approach was previously proposed by Runions [70] for the computation of a generalized spline.

A texture at a fixed $n \times n$ resolution is generated as follows. Let $\left\{u_{0}, u_{1}, \ldots, u_{n \times n}\right\}$ be the set of pixel locations we wish to compute a pixel value for, and let $w_{i}(p)$ be the weight function of a particular region with associated color $a_{i}$.

We allocate an array of evaluation points $A:\{0,1, \ldots, n \times n\} \rightarrow \mathbb{R}^{n}$, and another array $B:\{0,1, \ldots, n \times n\} \rightarrow \mathbb{R}$ to store the sum of weight values at each pixel location.

For each region, we iterate through pixel locations that are within the region 
support. For a pixel location $u_{j}$ within a region support we add $w_{i}\left(u_{j}\right) \cdot a_{i}$ to $A[j]$. At the same time, we also add $w_{i}\left(u_{j}\right)$ to $B[j]$. After we have iterated through all regions, we obtain

$$
\begin{aligned}
& A[j]=\sum_{i=0}^{N} w_{i}\left(u_{j}\right) a_{i} \\
& B[j]=\sum_{i=0}^{N} w_{i}\left(u_{j}\right)
\end{aligned}
$$

where $N$ is the number of regions in the texture. The color value of a pixel at location $u_{j}$ is obtained by dividing $A[j]$ by $B[j]$.

\subsubsection{Field Value Computation}

Another concern related to the performance of our algorithm is the efficient evaluation of the weight function $w_{i}(p)$ for a region. Evaluating $w_{i}(p)$ involves finding the smooth signed distance field for both the interior and exterior contour. For simple spot patterns, where the number of edges in the interior and exterior contours are small, we can directly determine the field value described in Section 3.6. However, for larger regions where a contour can be composed of many segments, direct computation of the smooth distance field can create both performance problems and numerical instabilities.

To improve performance, a line segment is included in the field calculation only within a local area of a region. Because closer line segments are weighted exponentially more in the distance calculation, we can disregard certain segments far enough away. The difficulty with this approach is that a cutoff distance would need to be defined relative to the distance of other line segments. Using a distance field approach allowed us to define texture values for all of $\mathbb{R}^{2}$, but can result in a large variation in the sizes of produced regions and thus large variations of influences from contour line segments.

A simple approach to restrict the area of a line segment contribution is to use the edges of the texture mesh. Let $\left(v_{1}, v_{2}\right)$ be a line segment that is part of a contour, and let $a$ be the length of the longest edge from either $v_{1}$ of $v_{2}$. We restrict the field calculation for the line segment to a square region with center $\left(v_{1}+v_{2}\right) / 2$ with side length $s=K \cdot a$ where $K$ is a constant used to control the smoothness of the resulting 
Table 6.1: Timing results for our algorithm for a binary texture $(400 \times 400)$.

\begin{tabular}{lcccc}
\hline Parameter $K$ & Point Spacing $d$ & Points & Edges & Time \\
\hline$K=1$ & 0.025 & 1109 & 3304 & $1.078 \mathrm{~s}$ \\
& 0.050 & 310 & 911 & $0.875 \mathrm{~s}$ \\
& 0.100 & 92 & 263 & $0.689 \mathrm{~s}$ \\
\hline$K=2$ & 0.025 & 1109 & 3304 & $2.922 \mathrm{~s}$ \\
& 0.050 & 310 & 911 & $2.469 \mathrm{~s}$ \\
& 0.100 & 92 & 263 & $2.016 \mathrm{~s}$ \\
\hline$K=4$ & 0.025 & 1109 & 3304 & $7.406 \mathrm{~s}$ \\
& 0.050 & 310 & 911 & $6.406 \mathrm{~s}$ \\
& 0.100 & 92 & 263 & $4.922 \mathrm{~s}$ \\
\hline
\end{tabular}

field. Larger values of $K$ increase the computation time but generate a smoother field.

Table 6.1 shows the timing results of our method for a texture created with a random binary labeling in a Poisson disk distribution, with blending radius parameter $r=d / 4$, and with different values for the parameter $K$. The timing results were generated on an Intel Core i5-5200U processor using a single-threaded unoptimized implementation. We note that the performance of our algorithm is linear in the size of the image. Table 6.2 shows timing results for a random binary texture generated at different resolutions.

Choosing a value for $K$ generally depends on the parameters used to generate the texture. For the binary texture that we generated our timing results for, we do not notice discontinuity artifacts beyond $K=2$.

\subsection{Summary}

This chapter discussed the rationale of our algorithm. We described how using a mesh allows us to group points together, and provides us a way to define region boundaries. Because each region is defined using a pair of contours, we can create smooth fields that limit bulge artifacts. Creating a field for each region then allows us to create a 
Table 6.2: Timing results for our algorithm for different resolutions.

\begin{tabular}{ccccc}
\hline Resolution & Point Spacing $d$ & Points & Edges & Time \\
\hline $200 \times 200$ & 0.025 & 1109 & 3304 & $1.078 \mathrm{~s}$ \\
& 0.050 & 310 & 911 & $0.875 \mathrm{~s}$ \\
& 0.100 & 92 & 263 & $0.689 \mathrm{~s}$ \\
\hline $400 \times 400$ & 0.025 & 1109 & 3304 & $2.922 \mathrm{~s}$ \\
& 0.050 & 310 & 911 & $2.469 \mathrm{~s}$ \\
\hline \multirow{2}{*}{$800 \times 800$} & 0.100 & 92 & 263 & $2.016 \mathrm{~s}$ \\
\hline 0.025 & 1109 & 3304 & $11.156 \mathrm{~s}$ \\
& 0.050 & 310 & 911 & $9.890 \mathrm{~s}$ \\
& 0.100 & 92 & 263 & $8.109 \mathrm{~s}$ \\
\hline
\end{tabular}

variety of effects in the texture.

We then described different performance concerns related to our algorithm. We showed how a pixel value could be evaluated at a random location in the texture, and how to generated a texture at a fixed resolution. We concluded by presenting the timing results of our algorithm for different resolutions and performance parameters. 


\section{Chapter 7}

\section{Conclusion}

\subsection{Summary}

We presented a method for generating smooth organic patterns using labeled points on a mesh. We used the edges of a mesh to group points together, and to define region boundaries in the texture. Each region is defined by blending together the distance fields of two different sets of boundary edges, and produces a field for the region that is everywhere smooth. We created a texture by composing the fields of different regions together.

Our method is distinguished from existing techniques by being able to produce a continuous, implicit representation of texture that can contain both smooth and irregularly shaped regions. Regions in the texture do not have to be convex, and can be smoothly blended with each other. Furthermore, the inputs to our method are closely related to the output texture, and different textures can be generated by varying how a mesh is constructed.

Partition of unity textures produce continuous textures, but region boundaries would appear bumpy and irregular. Reaction-diffusion textures produce a variety of organic texture, but each texture is discretized and has its input parameters be a set of partial differential equations.

There are several limitations to our method as well. In our approach, the gradient magnitude of a region distance field is not constant, which may cause variations in how regions are blended in the texture. Section 5.4.2 showed how the blending behaviour

of a stripe varied depending on the stripe width. In Section 5.3.2 we discussed how texture artifacts could be created depending on how the underlying mesh was defined.

Despite the above limitations, we have shown several types of smooth and irregular 
patterns. We were able to produce different spot, stripe, and Voronoi textures that resembled patterns found in the natural world.

\section{2 $\quad$ Future Work}

We believe there are several promising directions for future work. We have not yet considered applying our algorithm to generate surface texture, which is a necessarily requirement for texturing surfaces without warping and seam artifacts. Other possibilities for future work involve varying how the texture mesh is constructed, changing how a smooth distance field is defined, and producing complex multi-scale patterns.

Many of the patterns we generated were produced by distributing points in a Poisson disk distribution, and applying a Delaunay triangulation. An area of further exploration is to consider alternative mesh construction techniques. We have previously described how varying the distribution of points could produce non-stationary texture, but more interesting textures could be generated by using more sophisticated techniques. For example, we can consider using different mesh subdivision schemes to control the level of detail of the texture.

Another possibility for future work is to examine alternative approaches to constructing smooth distance fields. Our method blended smooth distance fields created using R-functions. Each smooth distance field was constructed by joining together a set of clipped implicit lines using R-equivalence. We can consider using other variations of constructing smooth distance fields by changing how implicit fields are created and combined together.

The more general problem we wish to solve is how to obtain smooth surfaces, given that surface boundaries are specified using discontinuous line segments. Ideally, a method would create surfaces that could be easily parameterized to provide control over the desired smoothness. Further work could involve investigating applying potential-based or convolution-based methods to construct smooth regions. Instead of blending between two sets of distance fields, one could investigate producing an everywhere smooth field using only a single set of line primitives.

We would also like to explore how to generate more complex patterns by combining multiple layers of texture together. We could consider generating textures at multiple levels of detail using different point densities, and combining these textures to form a complex pattern. Another approach is to have the point set and mesh in a layer be 
dependent on layers previously generated. Many patterns found in the natural world consist of features that have a large variety of size and shape.

Although we can extend our method in several ways, we have shown the usefulness of using smooth signed distance fields to produce texture. Our approach gave us a flexible and resolution-independent method to construct several different types of smooth and organic patterns. We believe further study of our work may lead to the generation of many other complex organic phenomena. 


\section{List of References}

[1] N. Ahuja and J.-H. Chuang, "Shape representation using a generalized potential field model," IEEE Trans. Pattern Anal. Mach. Intell., vol. 19, no. 2, pp. 169176, Feb. 1997.

[2] K. Anjyo, J. P. Lewis, and F. Pighin, "Scattered data interpolation for computer graphics," in ACM SIGGRAPH 2014 Courses, ser. SIGGRAPH '14. New York, NY, USA: ACM, 2014, pp. 27:1-27:69.

[3] B. R. D. Arajo, R. A. Redol, J. Armando, and P. Jorge, "Blobmaker: Free-form modelling with variational implicit surfaces," in In Proc. of the 12th Portuguese Computer Graphics Meeting, 2003, pp. 17-26.

[4] M. Ashikhmin, "Synthesizing natural textures," in Proceedings of the 2001 Symposium on Interactive 3D Graphics, ser. I3D '01. New York, NY, USA: ACM, 2001, pp. 217-226.

[5] T. Baert, "Eye," https://www.flickr.com/photos/60588229@N05/5725913600, 2011, [Online; accessed 03-09-16]. Licensed under CC BY-NC 2.0.

[6] J. B. Bard, "A model for generating aspects of zebra and other mammalian coat patterns," Journal of Theoretical Biology, vol. 93, no. 2, pp. 363 - 385, 1981.

[7] P. Batty, "Lizard," https://www.flickr.com/photos/ebatty/17453720, 2003, [Online; accessed 03-09-16] Licensed under CC BY-NC-SA 2.0.

[8] A. Belyaev, P.-A. Fayolle, and A. Pasko, "Technical note: Signed Lp-distance fields," Comput. Aided Des., vol. 45, no. 2, pp. 523-528, Feb. 2013.

[9] A. G. Belyaev and P.-A. Fayolle, "On variational and PDE-based distance function approximations," Computer Graphics Forum, 2015.

[10] Bemap, "Christmas tree worm on brain coral," https://www.flickr.com/photos/ 40626436@N00/4502224625, 2010, [Online; accessed 03-09-16]. Licensed under CC BY-NC 2.0.

[11] A. Biswas and V. Shapiro, "Approximate distance fields with non-vanishing gradients," Graph. Models, vol. 66, no. 3, pp. 133-159, May 2004.

[12] J. Bloomenthal, "Bulge elimination in convolution surfaces," Computer Graphics Forum, vol. 16, no. 1, pp. 31-41, 1997. 
[13] J. Bloomenthal and K. Shoemake, "Convolution surfaces," Computer Graphics (Proc. ACM SIGGRAPH 91), vol. 25, no. 4, pp. 251-256, Jul. 1991.

[14] J. Bloomenthal and B. Wyvill, Eds., Introduction to Implicit Surfaces. San Francisco, CA, USA: Morgan Kaufmann Publishers Inc., 1997.

[15] D. S. Broomhead and D. Lowe, "Multivariable functional interpolation and adaptive networks," Complex Systems 2, pp. 321-355, 1988.

[16] F. Canezin, G. Guennebaud, and L. Barthe, "SMI 2013: Adequate inner bound for geometric modeling with compact field functions," Computers $\&$ Graphics, vol. 37, no. 6, pp. 565-573, Oct. 2013.

[17] J. Caron and D. Mould, "Partition of unity parametrics for texture synthesis," in Proceedings of Graphics Interface 2013, ser. GI '13. Toronto, Ont., Canada, Canada: Canadian Information Processing Society, 2013, pp. 173-179.

[18] _ - "Texture synthesis using label assignment over a graph," Computers $\mathcal{E}$ Graphics, vol. 39, pp. $24-36,2014$.

[19] J. Cathey-Roberts, "Puffer fish," https://www.flickr.com/photos/ catheyroberts/2913111527, 2008, [Online; accessed 03-09-16]. Licensed under CC BY-NC 2.0.

[20] J. F. Clay, "Zebra," https://www.flickr.com/photos/jamesclay/1380022122, 2006, [Online; accessed 03-09-16] Licensed under CC BY-NC 2.0.

[21] —_, "Zebra," https://www.flickr.com/photos/jamesclay/1379943696, 2006, [Online; accessed 03-09-16]. Licensed under CC BY-NC 2.0.

[22] R. L. Cook and T. DeRose, "Wavelet noise," ACM Trans. Graph., vol. 24, no. 3, pp. 803-811, Jul. 2005.

[23] K. Crane, C. Weischedel, and M. Wardetzky, "Geodesics in heat: A new approach to computing distance based on heat flow," ACM Trans. Graph., vol. 32, no. 5, pp. 152:1-152:11, Oct. 2013.

[24] I. Dawned, "Toad," https://www.flickr.com/photos/ivydawned/8734243781, 2013, [Online; accessed 03-09-16]. Licensed under CC BY-NC-SA 2.0.

[25] D. S. Ebert, F. K. Musgrave, D. Peachey, K. Perlin, and S. Worley, Texturing and Modeling: A Procedural Approach, 3rd ed. San Francisco, CA, USA: Morgan Kaufmann Publishers Inc., 2002.

[26] A. A. Efros and W. T. Freeman, "Image quilting for texture synthesis and transfer," in Proceedings of the 28th Annual Conference on Computer Graphics and Interactive Techniques, ser. SIGGRAPH '01. New York, NY, USA: ACM, 2001, pp. 341-346.

[27] A. A. Efros and T. K. Leung, "Texture synthesis by non-parametric sampling," in Proceedings of the International Conference on Computer Vision-Volume 2 - Volume 2, ser. ICCV '99. Washington, DC, USA: IEEE Computer Society, 1999, pp. 1033-. 
[28] S. Epstein, "clown triggerfish," https://www.flickr.com/photos/serenae/ 9732242870, 2013, [Online; accessed 03-09-16]. Licensed under CC BY-NC-SA 2.0 .

[29] P. F. Felzenszwalb and D. P. Huttenlocher, "Distance transforms of sampled functions," Theory of Computing, vol. 8, no. 1, pp. 415-428, 2012.

[30] C. Galbraith, P. Prusinkiewicz, and B. Wyvill, "Modeling a murex cabritii sea shell with a structured implicit surface modeler," The Visual Computer, vol. 18, no. 2 , pp. $70-80,2002$.

[31] B. Galerne, A. Lagae, S. Lefebvre, and G. Drettakis, "Gabor noise by example," ACM Trans. Graph., vol. 31, no. 4, pp. 73:1-73:9, Jul. 2012.

[32] A. Gomes, I. Voiculescu, J. Jorge, B. Wyvill, and C. Galbraith, Implicit Curves and Surfaces: Mathematics, Data Structures and Algorithms, 1st ed. Springer Publishing Company, Incorporated, 2009.

[33] O. Gourmel, L. Barthe, M.-P. Cani, B. Wyvill, A. Bernhardt, M. Paulin, and H. Grasberger, "A gradient-based implicit blend," ACM Trans. Graph., vol. 32, no. 2, pp. 12:1-12:12, Apr. 2013.

[34] B. Gratwicke, "Oophaga pumilio," https://www.flickr.com/photos/ briangratwicke/14823348718, 2014, [Online; accessed 03-09-16]. Licensed under CC BY 2.0.

[35] J. Han, K. Zhou, L.-Y. Wei, M. Gong, H. Bao, X. Zhang, and B. Guo, "Fast example-based surface texture synthesis via discrete optimization," The Visual Computer, vol. 22, no. 9-11, pp. 918-925, 2006.

[36] T. R. Jones, "Efficient generation of poisson-disk sampling patterns," Journal of Graphics, GPU, and Game Tools, vol. 11, no. 2, pp. 27-36, 2006.

[37] T. Ju, "Scattered-data interpolation," in A Sampler of Useful Computational Tools for Applied Geometry, Computer Graphics, and Image Processing, 1st ed., D. Cohen-Or, Ed. Natick, MA, USA: A. K. Peters, Ltd., 2015, vol. 21, pp. $147-162$.

[38] O. Karpenko, J. F. Hughes, and R. Raskar, "Free-form sketching with variational implicit surfaces," Computer Graphics Forum, vol. 21, no. 3, pp. 585-594, 2002.

[39] D. Keats, "Emperor angelfish," https://www.flickr.com/photos/dkeats/ 6443170735, 2011, [Online; accessed 03-09-16]. Licensed under CC BY 2.0.

[40] J. Kirkland, "Ebv-4531.jpg," https://www.flickr.com/photos/27145142@N00/ 4223358735, 2009, [Online; accessed 03-09-16] Licensed under CC BY-NC-SA 2.0 .

[41] V. Kwatra, A. Schödl, I. Essa, G. Turk, and A. Bobick, "Graphcut textures: Image and video synthesis using graph cuts," ACM Trans. Graph., vol. 22, no. 3, pp. 277-286, Jul. 2003. 
[42] A. Lagae, S. Lefebvre, R. Cook, T. DeRose, G. Drettakis, D. S. Ebert, J. P. Lewis, K. Perlin, and M. Zwicker, "State of the art in procedural noise functions," in $E G$ 2010 - State of the Art Reports. Norrkoping, Sweden: Eurographics Association, May 2010.

[43] A. Lagae, S. Lefebvre, G. Drettakis, and P. Dutré, "Procedural noise using sparse Gabor convolution," ACM Trans. Graph., vol. 28, no. 3, pp. 54:1-54:10, Jul. 2009.

[44] A. Lagae, S. Lefebvre, and P. Dutré, "Improving Gabor noise," IEEE Transactions on Visualization and Computer Graphics, vol. 17, no. 8, pp. 1096-1107, August 2011.

[45] X. Latta, "Cutie patootie," https://www.flickr.com/photos/xanboozled/ 446681285, 2007, [Online; accessed 03-09-16]. Licensed under CC BY-NC-SA 2.0 .

[46] H. Ledoux and C. Gold, Developments in Spatial Data Handling: 11th International Symposium on Spatial Data Handling. Berlin, Heidelberg: Springer Berlin Heidelberg, 2005, ch. An Efficient Natural Neighbour Interpolation Algorithm for Geoscientific Modelling, pp. 97-108.

[47] S. Lefebvre and H. Hoppe, "Parallel controllable texture synthesis," ACM Trans. Graph., vol. 24, no. 3, pp. 777-786, Jul. 2005.

[48] J. P. Lewis, "Algorithms for solid noise synthesis," Computer Graphics (Proc. ACM SIGGRAPH 89), vol. 23, no. 3, pp. 263-270, Jul. 1989.

[49] J.-P. Lewis, "Texture synthesis for digital painting," Computer Graphics (Proc. ACM SIGGRAPH 84), vol. 18, no. 3, pp. 245-252, Jan. 1984.

[50] L. Liang, C. Liu, Y.-Q. Xu, B. Guo, and H.-Y. Shum, "Real-time texture synthesis by patch-based sampling," ACM Trans. Graph., vol. 20, no. 3, pp. 127-150, Jul. 2001.

[51] Lydia, "Butterfly," https://www.flickr.com/photos/lydz/171355654, 2006, [Online; accessed 03-09-16]. Licensed under CC BY-NC-SA 2.0.

[52] S. Mann, "A study of two implicit data interpolation schemes," Department of Computer Science, University of Waterloo, Tech. Rep. CS-2013-09, 2013.

[53] Marc, "Sleeping giraffe," https://www.flickr.com/photos/sumofmarc/ 9617372714, 2013, [Online; accessed 03-09-16]. Licensed under CC BY-NC-ND 2.0 .

[54] K. Mehlhorn, "A faster approximation algorithm for the Steiner problem in graphs," Information Processing Letters, vol. 27, no. 3, pp. 125 - 128, 1988.

[55] H. Meinhardt, Models of Biological Pattern Formation. Academic Press, London, 1982.

[56] A. Meintjes, "Leopard," https://www.flickr.com/photos/arnolouise/3195871485, 2009, [Online; accessed 03-09-16]. Licensed under CC BY-NC-SA 2.0. 
[57] B. S. Morse, T. S. Yoo, P. Rheingans, D. T. Chen, and K. R. Subramanian, "Interpolating implicit surfaces from scattered surface data using compactly supported radial basis functions," in ACM SIGGRAPH 2005 Courses, ser. SIGGRAPH '05. New York, NY, USA: ACM, 2005.

[58] J. D. Murray, "On pattern formation mechanisms for lepidopteran wing patterns and mammalian coat markings," Philosophical Transactions of the Royal Society of London B: Biological Sciences, vol. 295, no. 1078, pp. 473-496, 1981.

[59] Y. Ohtake, A. Belyaev, M. Alexa, G. Turk, and H.-P. Seidel, "Multi-level partition of unity implicits," in ACM SIGGRAPH 2003 Papers, ser. SIGGRAPH '03. New York, NY, USA: ACM, 2003, pp. 463-470.

[60] V. Ostromoukhov, C. Donohue, and P.-M. Jodoin, "Fast hierarchical importance sampling with blue noise properties," ACM Trans. Graph., vol. 23, no. 3, pp. 488-495, Aug. 2004.

[61] R. Palazzani, "Lizard," https://www.flickr.com/photos/veridiano3/ 13959669025, 2014, [Online; accessed 03-09-16]. Licensed under CC BY-NC-SA 2.0 .

[62] K. Perlin and E. M. Hoffert, "Hypertexture," Computer Graphics (Proc. ACM SIGGRAPH 89), pp. 253-262, 1989.

[63] K. Perlin, "An image synthesizer," Computer Graphics (Proc. ACM SIGGRAPH 85), pp. 287-296, 1985.

[64] — - "Improving noise," in Proceedings of the 29th Annual Conference on Computer Graphics and Interactive Techniques, ser. SIGGRAPH '02. New York, NY, USA: ACM, 2002, pp. 681-682.

[65] E. Praun, A. Finkelstein, and H. Hoppe, "Lapped textures," in Proceedings of the 27th Annual Conference on Computer Graphics and Interactive Techniques, ser. SIGGRAPH '00. New York, NY, USA: ACM Press/Addison-Wesley Publishing Co., 2000, pp. 465-470.

[66] Rastoney, "Blue poison dart frog," https://www.flickr.com/photos/ planmygreen/2690548176, 2008, [Online; accessed 03-09-16]. Licensed under CC BY-NC 2.0.

[67] M. Reitter, "smile," https://www.flickr.com/photos/m_reitter/2280508128, 2008, [Online; accessed 03-09-16]. Licensed under CC BY-NC-SA 2.0.

[68] R. J. Renka, "Multivariate interpolation of large sets of scattered data," $A C M$ Trans. Math. Softw., vol. 14, no. 2, pp. 139-148, Jun. 1988.

[69] A. Ricci, "A constructive geometry for computer graphics." Comput. J., vol. 16, no. 2, pp. 157-160, 1973.

[70] A. Runions and F. Samavati, "Partition of unity parametrics: a framework for meta-modeling," The Visual Computer, vol. 27, no. 6-8, pp. 495-505, 2011. 
[71] M. Sanchez, O. Fryazinov, P.-A. Fayolle, and A. Pasko, "Convolution filtering of continuous signed distance fields for polygonal meshes," Computer Graphics Forum, vol. 34, no. 6, pp. 277-288, 2015.

[72] B. Schachter and N. Ahuja, "Random pattern generation processes," Computer Graphics and Image Processing, vol. 10, no. 2, pp. 95-114, Jun. 1979.

[73] U. Schwarzbach, "Lionfish," https://www.flickr.com/photos/uwebkk/ 13773065613, 2014, [Online; accessed 03-09-16]. Licensed under CC BY-NC-SA 2.0 .

[74] V. Shapiro, "Semi-analytic geometry with R-functions," Acta Numerica, vol. 16, pp. 239-303, 52007.

[75] V. Shapiro and I. Tsukanov, "Implicit functions with guaranteed differential properties," in Proceedings of the Fifth ACM Symposium on Solid Modeling and Applications, ser. SMA '99. New York, NY, USA: ACM, 1999, pp. 258-269.

[76] D. Shepard, "A two-dimensional interpolation function for irregularly-spaced data," in Proceedings of the 1968 23rd ACM National Conference, ser. ACM '68. New York, NY, USA: ACM, 1968, pp. 517-524.

[77] P. Shirley and S. Marschner, Fundamentals of Computer Graphics, 3rd ed. Natick, MA, USA: A. K. Peters, Ltd., 2009.

[78] R. Sibson, "A brief description of natural neighbour interpolation," in Interpreting multivariate data, V. Barnett, Ed. John Wiley \& Sons, 1981, vol. 21, pp. $21-36$.

[79] C. Soler, M.-P. Cani, and A. Angelidis, "Hierarchical pattern mapping," $A C M$ Trans. Graph., vol. 21, no. 3, pp. 673-680, Jul. 2002.

[80] I. Sutton, "Spotted marsh frog," https://www.flickr.com/photos/22616984@ N07/5644930368, 2011, [Online; accessed 03-09-16]. Licensed under CC BY 2.0.

[81] J. Swick, "Al the leopard gecko," https://www.flickr.com/photos/simplyjessi/ 6328338235, 2010, [Online; accessed 03-09-16]. Licensed under CC BY 2.0.

[82] X. Tong, J. Zhang, L. Liu, X. Wang, B. Guo, and H.-Y. Shum, "Synthesis of bidirectional texture functions on arbitrary surfaces," in Proceedings of the 29th Annual Conference on Computer Graphics and Interactive Techniques, ser. SIGGRAPH '02. New York, NY, USA: ACM, 2002, pp. 665-672.

[83] A. M. Turing, "The chemical basis of morphogenesis," Philosophical Transactions of the Royal Society of London B: Biological Sciences, vol. 237, no. 641, pp. 37$72,1952$.

[84] G. Turk, "Generating textures on arbitrary surfaces using reaction-diffusion," Computer Graphics (Proc. ACM SIGGRAPH 91), vol. 25, no. 4, pp. 289-298, Jul. 1991.

[85] G. Turk and J. F. O'Brien, "Variational implicit surfaces," Georgia Institute of Technology, Tech. Rep. GITGVU-99-15, May 1999. 
[86] L. Wang, K. Zhou, Y. Yu, and B. Guo, "Vector solid textures," ACM Trans. Graph., vol. 29, no. 4, pp. 86:1-86:8, Jul. 2010.

[87] L.-Y. Wei, S. Lefebvre, V. Kwatra, and G. Turk, "State of the art in examplebased texture synthesis," in Eurographics 2009, State of the Art Report, EGSTAR. Eurographics Association, 2009.

[88] L.-Y. Wei and M. Levoy, "Fast texture synthesis using tree-structured vector quantization," in Proceedings of the 27th Annual Conference on Computer Graphics and Interactive Techniques, ser. SIGGRAPH '00. New York, NY, USA: ACM Press/Addison-Wesley Publishing Co., 2000, pp. 479-488.

[89] L. Wei and M. Levoy, "Order-independent texture synthesis," Computer Science Department, Stanford University, Tech. Rep. TR-2002-01, 2002.

[90] A. Witkin and M. Kass, "Reaction-diffusion textures," Computer Graphics (Proc. ACM SIGGRAPH 91), vol. 25, no. 4, pp. 299-308, Jul. 1991.

[91] S. Worley, "A cellular texture basis function," in Proceedings of the 23rd Annual Conference on Computer Graphics and Interactive Techniques, ser. SIGGRAPH '96. New York, NY, USA: ACM, 1996, pp. 291-294.

[92] Q. Wu and Y. Yu, "Feature matching and deformation for texture synthesis," ACM Trans. Graph., vol. 23, no. 3, pp. 364-367, Aug. 2004.

[93] B. Wyvill, A. Guy, and E. Galin, "Extending the CSG tree. Warping, blending and boolean operations in an implicit surface modeling system," Computer Graphics Forum, vol. 18, no. 2, pp. 149-158, 1999.

[94] L. Ying, A. Hertzmann, H. Biermann, and D. Zorin, "Texture and shape synthesis on surfaces," in Rendering Techniques 2001, ser. Eurographics, S. Gortler and K. Myszkowski, Eds. Springer Vienna, 2001, pp. 301-312.

[95] C. Zanni, P. Bares, A. Lagae, M. Quiblier, and M.-P. Cani, "Geometric details on skeleton-based implicit surfaces," in Eurographics 2012 - 33rd Annual Conference of the European Association for Computer Graphics, ser. Eurographics 2012 : Short Paper. Cagliari, Italy: Eurographics, May 2012, pp. 49-52. 